Supporting Information

ACS Sustainable Chemistry \& Engineering

\title{
Towards designing "sweet" ionic liquids containing a natural terpene moiety as effective wood preservatives
}

\author{
Joanna Feder-Kubis, ${ }^{*}{ }^{\dagger}$ Jadwiga Zabielska-Matejuk, ${ }^{\ddagger}$ Anna Stangierska, ${ }^{\ddagger}$ Piotr Przybylski,${ }^{\S}$ Johan \\ Jacquemin," and Monika Geppert-Rybczyńska ${ }^{\perp}$
}

\begin{abstract}
Wrocław University of Science and Technology, Faculty of Chemistry, Wybrzeże Wyspiańskiego 27, 50370 Wrocław, Poland

¥ Łukasiewicz Research Network-Wood Technology Institute, Winiarska 1, 60-654 Poznań, Poland

${ }^{\S}$ Adam Mickiewicz University in Poznań, Faculty of Chemistry, Uniwersytetu Poznańskiego 8, 61-614 Poznań, Poland

"Université de Tours, Laboratoire PCM2E, Parc Grandmont, 37200 TOURS, France

${ }^{\perp}$ University of Silesia, Institute of Chemistry, Szkolna 9, 40-006 Katowice, Poland

*Corresponding author at: Wrocław University of Science and Technology, Faculty of Chemistry, Wybrzeże Wyspiańskiego 27, 50-370 Wrocław, Poland. E-mail address: joanna.feder-kubis@pwr.edu.pl; Fax: +48 71 3280475; Tel: +48 3202975
\end{abstract}

Number of pages: 67

Number of figures: 75

Number of tables: 5

1. Materials and techniques $\quad$ S3-S4

2. Methods of analysis S4-S6

3. Spectral analysis of obtained ILs $\quad$ S7-S13

1. Table S1. Analysis of the ionic impurities in the studied "sweet" ionic liquids $\left[\mathrm{C}_{\mathrm{n}}-\mathrm{Im}-\mathrm{S} 14\right.$ $\left.\mathrm{CH}_{2} \mathrm{OMen}\right][\mathrm{Sacc}]$

2. Table S2. Abbreviation, yield, cationic active substance content, empirical formula, S15 elementary analysis calculation and found for the precursors, 3-alkyl-1-[(1R,2S,5R)-(-)menthoxymethyl]imidazolium chlorides

3. Table S3. Solubility $\quad$ S16

4. Table S4. Density of "sweet" ionic liquids [ $\left.\mathrm{C}_{\mathrm{n}}-\mathrm{Im}-\mathrm{CH}_{2} \mathrm{OMen}\right][\mathrm{Sacc}] \quad \mathrm{S} 17$

5. Table S5. Surface tension of "sweet" ionic liquids $\left[\mathrm{C}_{\mathrm{n}}-\mathrm{Im}-\mathrm{CH}_{2} \mathrm{OMen}\right][\mathrm{Sacc}] \quad \mathrm{S} 18$ 
6. Fig. S1 - Fig. S2. Surface tension of "sweet" ionic liquids $\left[\mathrm{C}_{\mathrm{n}}-\mathrm{Im}-\mathrm{CH}_{2} \mathrm{OMen}\right][\mathrm{Sacc}]$ and S19-S20 comparisons with other ionic liquids

7. Fig. S3a. Photos of beech wood and Scots pine wood $\quad$ S21

$\begin{array}{ll}\text { 8. Fig. S3b. Profiles of beech wood and pine wood } & \text { S21 }\end{array}$

9. Fig. S4. Photo of beech wood and pine wood before measurements (from CCD camera) S22

10. Fig. S5 - Fig. S9. Photo of drop of C8 saccharinate at various temperatures and various S22-S23 wood materials

11. Fig. S10 - Fig. S11. Photo of drop of C6 saccharinate at various temperatures on glass $\quad$ S24

12. Fig. S12 - Fig. S14. Contact angle of "sweet" ionic liquids on various materials $\quad$ S25-S26

13. Fig. S15 - Fig. S75. NMR spectra of the discussed CILs in $\mathrm{CDCl}_{3} \quad$ S27-S66

$\begin{array}{ll}\text { 14. References } & \text { S67 }\end{array}$ 


\section{Materials and techniques.}

The materials used were sourced as follows: (1R,2S,5R)-(-)-menthol (99\%), paraformaldehyde (powder, 95\%), sodium (cubes, in mineral oil, 99.9\% trace metals basis), imidazole ( $\geq 99 \%)$, 1-methylimidazole (99\%), 1-butylimidazole (98\%), bromoethane ( $\geq 99 \%), 1$ bromopropane (99\%), 1-bromopentane (98\%), 1-bromohexane (98\%), 1-bromoheptane (99\%), 1bromooctane (99\%), 1-bromononane (98\%), 1-bromodecane (98\%), 1-bromoundecane (98\%), 1bromododecane (97\%), sodium saccharinate (hydrate, $\geq 99 \%$ ), hydrochloric acid (35-38\%), and sulphuric acid ( $\geq 96 \%$ ) were purchased from Merck. The drying agents, such as sodium sulphate (anhydrous, pure), and diphosphorus pentoxide (powder, anhydrous, $\geq 98 \%$ ) were provided by Alchem (Gliwice, Poland).

Deuterated chloroform $\left(\mathrm{CDCl}_{3}\right)$ purchased from Merck was used for NMR analysis. All solvents were purchased from the commercial suppliers Merck and Fluka and were dried before use. All reagents were dried and purified according to common procedures before use.

The structure and purity of each synthesized substance was confirmed by spectral analysis. Elemental analyses were carried out for all of the synthesized substances using a VARIO EL-III. The ${ }^{1} \mathrm{H}$ NMR and ${ }^{13} \mathrm{C}$ NMR spectra were recorded in $\mathrm{CDCl}_{3}$ as the solvent on a Bruker DRX instrument with tetramethylsilane as the standard (at 600 and $151 \mathrm{MHz}$, respectively). Melting points were determined with an electrothermal digital melting point apparatus model JA 9100 (temperature resolution $\pm 0.1{ }^{\circ} \mathrm{C}$; accuracy $\pm 1 \%$; ramp rate of $1.0^{\circ} \mathrm{C} / \mathrm{min}$ ). The type and shape of the crystals were analyzed with an optical microscope (Axiolmager M1m Zeiss) in reflection mode. The specific rotations at $578 \mathrm{~nm}$ at 1000 mbar were measured at $25^{\circ} \mathrm{C}$ using an Optical Activity Ltd. model AA-5 automatic polarimeter (resolution $\pm 0.01^{\circ}$, reproducibility $\pm 0.01^{\circ}$, accuracy \pm $0.01^{\circ}$, temperature-probe measurement accuracy $\pm 0.1^{\circ} \mathrm{C}$; the equipment provide four results for each measurement). The refractive index at $20{ }^{\circ} \mathrm{C}$ at $1000 \mathrm{mbar}, \mathrm{n}_{\mathrm{D}}{ }^{20}$, for the studied liquid salts with saccharinate anions (from butyl to dodecyl substituents: 3d-3l) was measured with an RX$5000 \alpha$ automatic digital refractometer (Atago) with an uncertainty of $\pm 0.00004 \mathrm{n}_{\mathrm{D}}$ units and a temperature-probe measurement accuracy of $\pm 0.1^{\circ} \mathrm{C}$. Before each measurement, the refractive index of pure distilled water was measured and compared with the known literature value $\left(\mathrm{n}_{\mathrm{D}}{ }^{20}=\right.$ $1.33300)$. 
Densities were measured instrumentally using the vibrating-tube method (Anton Paar, DMA 5000 densimeter). The uncertainty of the density measurements was $\pm 0.00002 \mathrm{~g}^{\circ} \mathrm{cm}^{-3}$; however, in our case, because of the high viscosities of the samples $\left(>0.008 \mathrm{~m}^{2} \mathrm{~s}^{-1}\right.$ at $\left.35{ }^{\circ} \mathrm{C}\right)$, the uncertainty was better than $0.0001 \mathrm{~g} \cdot \mathrm{cm}^{-3}$. For the aforementioned reason, the temperature range for the density measurements was $30{ }^{\circ} \mathrm{C}$ (or $35{ }^{\circ} \mathrm{C}$ for the $\mathrm{C}_{9}$ homologue) to $65{ }^{\circ} \mathrm{C}$ with steps of $5{ }^{\circ} \mathrm{C}$. The apparatus was calibrated using deionized water. After each measurement, the densimeter was washed using methanol (if necessary) and acetone and dried. All of the investigated CILs were dried at $t \approx 50{ }^{\circ} \mathrm{C}$ under vacuum $(\sim 5 \mathrm{mbar})$ for a few days.

\section{Methods of analysis.}

Karl Fischer analysis. The water content in each of the samples was determined by means of a TitroLine 7500 Karl Fischer trace coulometer (SI Analytics GmbH, Germany); none of the samples contained more than $0.13 \%$ water by mass. The relative uncertainty in the water determination by this method ${ }^{1}$ is approximately $1 \%$. The results of the water determinations of the samples after drying are listed in Table 2. To minimize contact with air moisture between the individual experiments, the studied CILs were stored under argon in vessels sealed with septa. Prior to the experiments, to make the samples fluid, they were heated to $50^{\circ} \mathrm{C}$.

IC analysis. Ion chromatography (IC) analyses for both cations $\left(\mathrm{Li}^{+}, \mathrm{Na}^{+}, \mathrm{NH}_{4}^{+}, \mathrm{K}^{+}, \mathrm{Ca}^{2+}\right.$, and $\left.\mathrm{Mg}^{2+}\right)$ and anions $\left(\mathrm{F}^{-}, \mathrm{Cl}^{-}, \mathrm{Br}^{-}, \mathrm{SO}_{4}{ }^{2-}, \mathrm{NO}_{3}{ }^{-}, \mathrm{NO}_{2}{ }^{-}\right.$, and $\left.\mathrm{H}_{2} \mathrm{PO}_{4}^{-}\right)$were carried out in parallel using a Dionex 5000 system to estimate the purity of the studied CILs. An IonPac AS11-HC $2 \times 250$ $\mathrm{mm}^{2}$ column was used for anions with hydroxide as the eluent, and an IonPac CS18 $2 \times 250 \mathrm{~mm}^{2}$ column was used for cations with methane sulfonic acid solution as the eluent. Both the anion and cation systems were equipped with electrochemical suppressors. External calibration was performed using NIST-traceable calibration solutions. The relative uncertainty in the ion concentration determinations was $20 \%$, with a detection limit of $0.001 \mathrm{ppm}$. The chloride and sodium contents and total purities of the discussed "sweet" ILs are summarized in Table 2 in the main manuscript; the amounts of all ionic impurities are listed in Table S1. 
Thermal analysis. Melting point and glass transition analyses were conducted using the differential scanning calorimetry (DSC) method in a STARe System (Mettler-Toledo) cooled with a Huber TC100 immersion cooler. The temperature and cell constants of the calorimeter were calibrated using high purity indium (melting temperature: $156.6^{\circ} \mathrm{C}$; specific enthalpy of melting: $\left.28.71 \mathrm{~J} \mathrm{~g}^{-1}\right)$. The samples weighed between 6 and $15 \mathrm{mg}$, were enclosed in aluminium pans and were stored under argon (flow rate: $20 \mathrm{~mL} / \mathrm{min}$ ). In the first heating cycle the heating ramp was set from $25^{\circ} \mathrm{C}$ to $120^{\circ} \mathrm{C}$ with a heating rate of $10{ }^{\circ} \mathrm{C} / \mathrm{min}$. The samples were held for $15 \mathrm{~min}$ at the final temperature. In the next step, samples were cooled from $120^{\circ} \mathrm{C}$ to $-80^{\circ} \mathrm{C}$ at a cooling rate of $10{ }^{\circ} \mathrm{C} / \mathrm{min}$; they were then maintained at constant temperature $\left(-80^{\circ} \mathrm{C}\right)$ for $5 \mathrm{~min}$. The last step was heating the sample from $-80{ }^{\circ} \mathrm{C}$ to $120^{\circ} \mathrm{C}$ at a heating rate of $10^{\circ} \mathrm{C} / \mathrm{min}$.

Thermogravimetric analyses. Thermogravimetric analysis was performed on a TGA Q50 from TA Instruments. The samples weighed between 5 and $10 \mathrm{mg}$ and were heated under a nitrogen atmosphere from $25^{\circ} \mathrm{C}$ to $500{ }^{\circ} \mathrm{C}$ at a heating rate of $10^{\circ} \mathrm{C} / \mathrm{min}$ with a $10 \mathrm{~min}$ isothermal hold at $80{ }^{\circ} \mathrm{C}$. This isothermal step was intended to volatilize any remaining water and possible volatile impurities remaining in the samples. From the thermal decomposition profile of each material, the onset temperature for the decomposition of the first $5 \%$ of the sample ( $\mathrm{T}_{5 \%} \%$ nset $)$ and the regular onset temperature for the decomposition ( $\mathrm{T}_{\text {onset }}$ ) either for the whole sample or for each of the consecutive steps in a multistep decomposition were determined.

The cationic active substance content. The cationic active content was assayed by a direct two-phase titration as follows: (i) for salts with a molar mass less than $500 \mathrm{~g} \cdot \mathrm{mol}^{-1}(\mathbf{2 a - 2 l}$ and 3a3e), the EN ISO 2871-2 procedure ${ }^{2}$ was used, and (ii) salts with a higher molar mass (3f-3l) were analysed according to the EN ISO $2871-1$ technique. $^{3}$ The method involves titration in a waterchloroform system (in the case of salts completely soluble in water; 2a-2l) or in a water-methanolchloroform system (for compounds with limited solubility in water; 3a-31) using the proper quaternary ammonium salt (in our case, quaternary imidazolium salts 2a-2l and 3a-31). Standard sodium dodecylsulphate(VI) solution in the presence of the mixed indicator dimidium bromide (CAS: 518-67-2) was used to determine the cationic active substances, and sulphan blue indicator (CAS: 129-17-9) was used to determine the anionic active substances. 
Solubility. The solubility of each of the obtained CILs was investigated according to the procedure outlined in Vogel's Textbook of Practical Organic Chemistry. ${ }^{4}$ The measurements for all the saccharinate salts $(3 \mathbf{a}-3 \mathrm{l})$ were carried out at $25^{\circ} \mathrm{C}\left( \pm 0.5^{\circ} \mathrm{C}\right)$ and at $50{ }^{\circ} \mathrm{C}\left( \pm 0.5^{\circ} \mathrm{C}\right)$ under ambient pressure using common solvents. The phrase complete solubility refers to those salts for which $0.1 \mathrm{~g}( \pm 0.01 \mathrm{~g})$ of solid is soluble in $1 \mathrm{~mL}( \pm 0.01 \mathrm{~mL})$ of solvent, whereas limited solubility refers to those salts for which $0.1 \mathrm{~g}( \pm 0.01 \mathrm{~g})$ of solid is soluble in $3 \mathrm{~mL}( \pm 0.1 \mathrm{~mL})$ of a given solvent. Insoluble indicates that $0.1 \mathrm{~g}( \pm 0.01 \mathrm{~g})$ of the salt was not soluble in $3 \mathrm{~mL} \pm 0.1 \mathrm{~mL}$ of the solvent.

Surface tension and contact angle measurements. The surface tension, $\gamma$, (by the pendant drop method), and contact angle, $\theta$, measurements were performed with a DSA 100S Krüss apparatus (Drop Shape Analysis software). The instrumental details and the experimental procedures have been described previously., ${ }^{5,6}$ The surface tension was measured in the general temperature range $30 \leq t \leq 60{ }^{\circ} \mathrm{C}$ in increments of $5^{\circ} \mathrm{C}$. For each temperature, the measuring procedure was repeated several times. The general uncertainty of the method for measuring surface tension is given by the manufacturer as $0.1 \mathrm{mN} \cdot \mathrm{m}^{-1}$; however, in our case, the standard deviation of the mean value (from 7-10 points) was approximately $0.05 \mathrm{mN} \cdot \mathrm{m}^{-1}$. The contact angle for each IL was obtained as the average of many independent measurements at $30{ }^{\circ} \mathrm{C}$ for the $\mathrm{C}_{6}, \mathrm{C}_{8}, \mathrm{C}_{10}$ and $\mathrm{C}_{12}$ homologues and at $35^{\circ} \mathrm{C}$ for $\mathrm{C}_{4}$ (to provide for better fluidity of the sample). Two different woods (Scots pine wood and beech wood) and microscopic glass (BK7) were chosen as surfaces on which to measure $\theta$. For contact angle measurements on the woods, a few different pieces were used for each IL and several measurements were made at different places on these surfaces. The glass surfaces for the measurements were rinsed with water containing detergent, then with distilled water and finally with acetone. After drying, the surfaces were stored in a vessel with a relative humidity of approximately $40 \%$. The wood surfaces were not prepared in a particular manner before measurements; however, they were stored in a vessel with a relative humidity of approximately $40 \%$, as in the experimental contact angle investigations. The precision of the contact angle measurements was $0.01^{\circ}$, but the estimated uncertainty was from 0.5 to $1.0^{\circ}$. 


\section{Spectral analysis of obtained ILs.}

NMR characterization. The structures of the saccharinate-based CILs were identified by ${ }^{1} \mathrm{H}$ and ${ }^{13} \mathrm{C}$ NMR spectroscopy. As shown in the spectra presented in the ESI (pages: S27-S66), the experimental NMR signals were consistent with the proposed structures.

The quaternization of chloromethyl $(1 R, 2 S, 5 R)-(-)$-menthyl ether with 1-alkylimidazole resulted in diastereotopic protons (protons of the $\mathrm{N}^{-} \mathrm{CH}_{2}-\mathrm{O}$ group, number 11 based on the numbering scheme), and the signals of these protons were characteristic for all of the studied salts; $e . g$., in the ${ }^{1} \mathrm{H}$ NMR spectrum of 3a, they appeared at $5.60 \mathrm{ppm}$ and $5.80 \mathrm{ppm}(\mathrm{d}, J=10,6 \mathrm{~Hz}, 2 \mathrm{H}, \mathrm{AB}$ system, H11). The multiplets at 7.51-7.53 ppm and 7.71-7.74 ppm in the ${ }^{1} \mathrm{H}$ NMR spectrum assigned to the aromatic groups (no: $\mathrm{H} 27 \div \mathrm{H} 30$ ) confirmed the presence of the saccharinate anion. The atoms were numbered according to the structure below, and the numbering scheme is consistent with the numbering of optically active $(1 R, 2 S, 5 R)-(-)$-menthol:
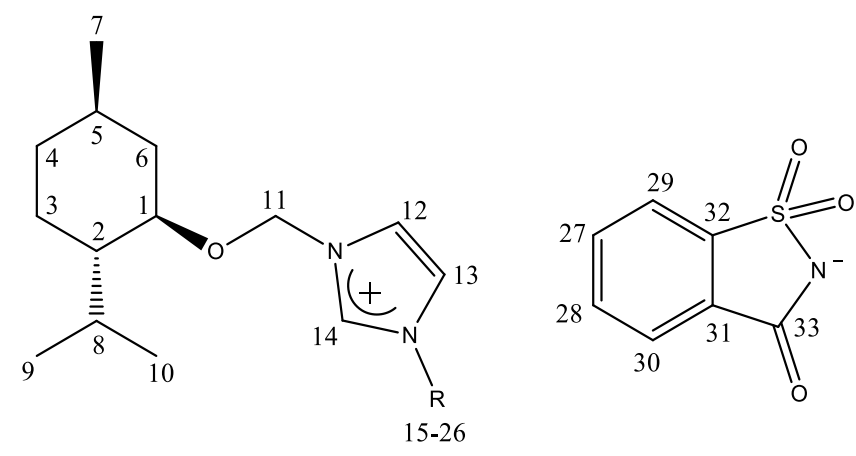

Elemental analysis. Elemental analysis helped confirm the identities and purities of the synthesized saccharinate-based CILs; for all compounds, the percentage differences between the experimental and calculation data did not exceed $0.21 \%$ for $\mathrm{C}, 0.18 \%$ for $\mathrm{H}$, and $0.20 \%$ for $\mathrm{N}$.

\section{1-[(1R,2S,5R)-(-)-Menthoxymethyl]-3-methylimidazolium saccharinate [ $\mathrm{C}_{1}-\mathrm{Im}-\mathrm{CH}_{2} \mathrm{O}$ -} Men][Sacc] (3a): Yield: 99.5\%, $0.0398 \mathrm{~mol}, 17.260 \mathrm{~g} .{ }^{1} \mathrm{H} \mathrm{NMR}\left(600 \mathrm{MHz}, \mathrm{CDCl}_{3}, 298 \mathrm{~K}\right.$ ) $\delta / \mathrm{ppm}$ 0.44 (d, $J=7.0 \mathrm{~Hz}, 3 \mathrm{H}, \mathrm{H} 9$ or H10), 0.72-0.85 (m, 9H, Ha-4, H7, H9 or H10, Ha-6 and Ha-3), 1.14-1.18 (m, 1H, H2), 1.22-1.25 (m, 1H, H5), 1.51-1.55 (m, 2H, Hb-3 and Hb-4), 1.88 (sept d, $J^{1,3}$ $\left.=7.0 \mathrm{~Hz}, J^{1,2}=2.3 \mathrm{~Hz}, 1 \mathrm{H}, \mathrm{H} 8\right), 2.01-2.04(\mathrm{~m}, 1 \mathrm{H}, \mathrm{Hb}-6), 3.28\left(\mathrm{td}, J^{1,3}=10,6 \mathrm{~Hz}, J^{1,2}=4.3 \mathrm{~Hz}\right.$, 
1H, H1), 4.08 (s, 3H, H15), 5.60 and 5.80 (d, $J=10.6 \mathrm{~Hz}, 2 \mathrm{H}, \mathrm{AB}$ system, H11), 7.30 (s, 1H, H12), 7.34-7.35 (m, 1H, H13), 7.51-7.53 (m, 2H, H27 and H28), 7.71-7.74 (m, 2H, H29 and H30), 10.24 (s, 1H, H14); ${ }^{13} \mathrm{C}$ NMR (300 MHz, $\mathrm{CDCl}_{3}, 298 \mathrm{~K}$ ) 8/ppm 15.53 (C9 or C10), 20.95 (C7), 22.03 (C9 or C10), 22.79 (C3), 25.42 (C8), 31.09 (C5), 34.04 (C4), 36.69 (C15), 40.13 (C6), 47.76 (C2), 76.85 (C1), 79.67 (C11), 119.75 (C29), 121.06 (C12), 123.14 (C30), 123.51 (C13), 131.36 (C27), 131.93 (C28), 134.77 (C14), 138.44 (C31), 144.64 (C32), 170.48 (C33). Elemental analysis: calc. (\%) for $\mathrm{C}_{22} \mathrm{H}_{31} \mathrm{O}_{4} \mathrm{~N}_{3} \mathrm{~S}$ (433.56): C 60.94, H 7.21, N 9.69, found: C 61.05, H 7.31, N 9.57.

3-Ethyl-1-[(1R,2S,5R)-(-)-menthoxymethyl]imidazolium saccharinate $\left[\mathrm{C}_{2}-\mathrm{Im}-\mathrm{CH}_{2} \mathrm{O}\right.$ Men][Sacc] (3b): Yield: 99.0\%, $0.0396 \mathrm{~mol}, 17.725 \mathrm{~g} .{ }^{1} \mathrm{H}$ NMR (600 MHz, $\left.\mathrm{CDCl}_{3}, 298 \mathrm{~K}\right) \delta / \mathrm{ppm}$ 0.41 (d, $J=7.0 \mathrm{~Hz}, 3 \mathrm{H}, \mathrm{H} 9$ or H10), 0.70-0.89 (m, 9H, Ha-4, H7, H9 or H10, Ha-6 and Ha-3), 1.13-1.18 (m, 1H, H2), 1.21-1.27 (m, 1H, H5), 1.50-1.57 (m, 5H, Hb-3, Hb-4 and H16), 1.87-2.18 (m, 2H, H8 and Hb-6), $3.30\left(\mathrm{td}, J^{1,3}=10.6 \mathrm{~Hz}, J^{1,2}=4.3 \mathrm{~Hz}, 1 \mathrm{H}, \mathrm{H1}\right), 4.37-4.43(\mathrm{~m}, 2 \mathrm{H}, \mathrm{H} 15)$, 5.61 and 5.85 (d, $J=10.6 \mathrm{~Hz}, 2 \mathrm{H}, \mathrm{AB}$ system, H11), 7.32 (s, 1H, H12), 7.37 (s, 1H, H13), 7.507.53 (m, 2H, H27 and H28), 7.70-7.74 (m, 2H, H29 and H30), 10.38 (s, 1H, H14); ${ }^{13} \mathrm{C}$ NMR (300

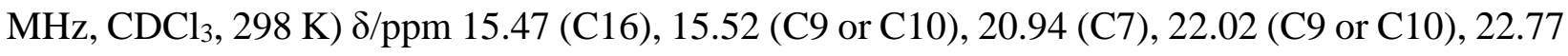
(C3), 25.45 (C8), 31.05 (C5), 34.05 (C4), 40.17 (C6), 45.46 (C15), 47.70 (C2), 76.85 (C1), 79.66 (C11), 119.75 (C29), 121.27 (C12), 121.69 (C13), 123.14 (C30), 131.33 (C27), 131.89 (C28), 134.78 (C14), 137.75 (C31), 144.69 (C32), 170.49 (C33). Elemental analysis: calc. (\%) for $\mathrm{C}_{23} \mathrm{H}_{33} \mathrm{O}_{4} \mathrm{~N}_{3} \mathrm{~S}$ (447.59): $\mathrm{C} 61.72, \mathrm{H}$ 7.43, $\mathrm{N}$ 9.39, found: $\mathrm{C} 61.61, \mathrm{H}$ 7.58, $\mathrm{N} 9.44$.

1-[(1R,2S,5R)-(-)-Menthoxymethyl]-3-propylimidazolium saccharinate [ $\mathrm{C}_{3}$-Im- $\mathrm{CH}_{2} \mathrm{O}$ Men][Sacc] (3c): Yield: 99.0\%, $0.0396 \mathrm{~mol}, 18.280 \mathrm{~g} .{ }^{1} \mathrm{H} \mathrm{NMR}\left(600 \mathrm{MHz}, \mathrm{CDCl}_{3}, 298 \mathrm{~K}\right.$ ) $\delta / \mathrm{ppm}$ 0.44 (d, $J=7.0 \mathrm{~Hz}, 3 \mathrm{H}, \mathrm{H} 9$ or H10), 0.70-0.89 (m, 12H, Ha-4, H7, H9 or H10, Ha-6, Ha-3 and H17), 1.19-1.24 (m, 1H, H2), 1.27-1.33 (m, 1H, H5), 1.57-1.61 (m, 2H, Hb-3, Hb-4), 1.92-1.99 (m, 3H, H8 and H16), 2.06-2.08 (m, 1H, Hb-6), 3.37 (td, $J^{1,3}=10,6 \mathrm{~Hz}, J^{1,2}=4,3 \mathrm{~Hz}, 1 \mathrm{H}, \mathrm{H1}$ ), 4.35-4.37 (m, 2H, H15), 5.68 and 5.92 (d, $J=10,7 \mathrm{~Hz}, 2 \mathrm{H}, \mathrm{AB}$ system, H11), 7.33 (s, 1H, H12), 7.43 (s, 1H, H13), 7.56-7.57 (m, 2H, H27 and H28), 7.77-7.79 (m, 2H, H29 and H30), 10.39 (s, 1H, H14); ${ }^{13} \mathrm{C}$ NMR (300 MHz, $\mathrm{CDCl}_{3}, 298 \mathrm{~K}$ ) 8/ppm 10.64 (C17), 15.55 (C9 or C10), 20.93 (C7), 22.01 (C9 or C10), 22.80 (C3), 23.63 (C16), 25.47 (C8), 31.05 (C5), 34.05 (C4), 40.23 (C6), 47.71 (C2), 51.69 (C15), 76.85 (C1), 79.82 (C11), 119.76 (C29), 121.16 (C12), 122.01 (C13), 123.12 (C30), 131.30 (C27), 131.86 (C28), 134.82 (C14), 138.13 (C31), 144.72 (C32), 170.49 (C33). 
Elemental analysis: calc. (\%) for $\mathrm{C}_{24} \mathrm{H}_{35} \mathrm{O}_{4} \mathrm{~N}_{3} \mathrm{~S}$ (461.62): C 62.44, H 7.64, N 9.10, found: C 62.56, H 7.79, N 9.01.

3-Butyl-1-[(1R,2S,5R)-(-)-menthoxymethyl]imidazolium saccharinate $\left[\mathrm{C}_{4}-\mathrm{Im}-\mathrm{CH}_{2} \mathrm{O}\right.$ Men][Sacc] (3d): Yield: 99.5\%, $0.0398 \mathrm{~mol}, 18.930 \mathrm{~g} .{ }^{1} \mathrm{H}$ NMR (600 MHz, $\mathrm{CDCl}_{3}, 298 \mathrm{~K}$ ) $\delta / \mathrm{ppm}$ 0.44 (d, $J=7.0 \mathrm{~Hz}, 3 \mathrm{H}, \mathrm{H} 9$ or H10), 0.70-0.84 (m, 9H, Ha-4, H7, H9 or H10, Ha-6 and Ha-3), 0.85-0.91 (m, 3H, H18), 1.15-1.19 (m, 1H, H2), 1.25-1.28 (m, 1H, H5), 1.33 (sex, 2H, H17), 1.521.56 (m, 2H, Hb-3, Hb-4), 1.82-1.90 (m, 3H, H8 and H16), 2.02-2.04 (m, 1H, Hb-6), 3.32 (td, $J^{1,3}$ $\left.=10,6 \mathrm{~Hz}, J^{1,2}=4,3 \mathrm{~Hz}, 1 \mathrm{H}, \mathrm{H} 1\right), 4.33(\mathrm{t}, J=7.3 \mathrm{~Hz}, 2 \mathrm{H}, \mathrm{H} 15), 5.63$ and $5.87(\mathrm{~d}, J=10,7 \mathrm{~Hz}, 2 \mathrm{H}$, AB system, H11), 7.25 (s, 1H, H12), 7.38 (s, 1H, H13), 7.50-7.53 (m, 2H, H27 and H28), 7.71-

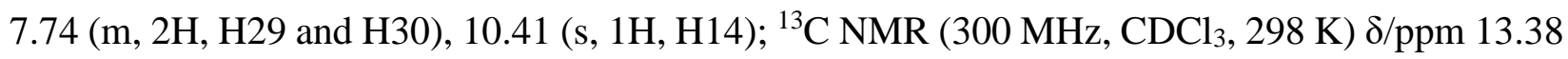
(C18), 15.53 (C9 or C10), 19.43 (C17), 20.94 (C7), 22.01 (C9 or C10), 22.81 (C3), 25.48 (C8), 31.06 (C5), 32.11 (C16), 34.05 (C4), 40.25 (C6), 47.72 (C2), 50.04 (C15), 76.85 (C1), 79.84 (C11), 119.77 (C29), 121.16 (C12), 121.91 (C13), 123.13 (C30), 131.30 (C27), 131.85 (C28), 134.79 (C14), 138.11 (C31), 144.72 (C32), 170.50 (C33). Elemental analysis: calc. (\%) for $\mathrm{C}_{25} \mathrm{H}_{37} \mathrm{O}_{4} \mathrm{~N}_{3} \mathrm{~S}$ (475.64): C 63.13, H 7.84, N 8.83, found: C 63.07, H 7.96, N 8.92.

1-[(1R,2S,5R)-(-)-Menthoxymethyl]-3-pentylimidazolium saccharinate [ $\mathrm{C}_{5}-\mathrm{Im}-\mathrm{CH}_{2} \mathrm{O}$ Men][Sacc] (3e): Yield: 98.0\%, $0.0392 \mathrm{~mol}, 19.195 \mathrm{~g} .{ }^{1} \mathrm{H}$ NMR (600 MHz, CDCl 3 , $298 \mathrm{~K}$ ) $\delta / \mathrm{ppm}$ 0.44 (d, $J=7.0 \mathrm{~Hz}, 3 \mathrm{H}, \mathrm{H} 9$ or H10), 0.73-0.90 (m, 12H, Ha-4, H7, H9 or H10, Ha-6, Ha-3 and H19), 1.15-1.31 (m, 6H, H2, H5, H17 and H18), 1.52-1.56 (m, 2H, Hb-3, Hb-4), 1.84-1.895 (m, $3 \mathrm{H}, \mathrm{H} 8$ and H16), 2.03-2.05 (m, 1H, Hb-6), $3.32\left(\mathrm{td}, J^{1,3}=10,6 \mathrm{~Hz}, J^{1,2}=4,3 \mathrm{~Hz}, 1 \mathrm{H}, \mathrm{H} 1\right), 4.31-$ 4.35 (m, 2H, H15), 5.63 and 5.87 (d, $J=10,7 \mathrm{~Hz}, 2 \mathrm{H}, \mathrm{AB}$ system, H11), 7.25 (s, 1H, H12), 7.38 (s, 1H, H13), 7.50-7.53 (m, 2H, H27 and H28), 7.71-7.73 (m, 2H, H29 and H30), 10.39 (s, 1H, H14); ${ }^{13} \mathrm{C}$ NMR (300 MHz, $\mathrm{CDCl}_{3}, 298 \mathrm{~K}$ ) 8/ppm 13.78 (C19), 15.55 (C9 or C10), 20.94 (C7), 22.02 (C9 or C10 and C18), 22.79 (C3), 25.47 (C8), 28.20 (C17), 29.90 (C16), 31.06 (C5), 34.06 (C4), 40.22 (C6), 47.71 (C2), 50.28 (C15), 76.80 (C1), 79.78 (C11), 119.79 (C29), 121.12 (C12), 121.86 (C13), 123.13 (C30), 131.31 (C27), 131.86 (C28), 134.77 (C14), 138.15 (C31), 144.71 (C32), 170.51 (C33). Elemental analysis: calc. (\%) for $\mathrm{C}_{26} \mathrm{H}_{39} \mathrm{O}_{4} \mathrm{~N}_{3} \mathrm{~S}$ (489.67): $\mathrm{C} 63.77, \mathrm{H} 8.03, \mathrm{~N}$ 8.58, found: C 63.86, H 8.19, N 8.42. 


\section{3-Hexyl-1-[(1R,2S,5R)-(-)-menthoxymethyl]imidazolium saccharinate [ $\mathrm{C}_{6}-\mathrm{Im}-\mathrm{CH}_{2} \mathrm{O}$ -}

Men][Sacc] (3f): Yield: 98.5\%, $0.0394 \mathrm{~mol}, 19.846 \mathrm{~g} .{ }^{1} \mathrm{H}$ NMR (600 MHz, $\left.\mathrm{CDCl}_{3}, 298 \mathrm{~K}\right) \delta / \mathrm{ppm}$ 0.44 (d, $J=7.0 \mathrm{~Hz}, 3 \mathrm{H}, \mathrm{H} 9$ or H10), 0.73-0.88 (m, 12H, Ha-4, H7, H9 or H10, Ha-6, Ha-3 and H20), 1.15-1.30 (m, 8H, H2, H5, H17, H18 and H19), 1.52-1.56 (m, 2H, Hb-3, Hb-4), 1.83-1.90 (m, 3H, H8 and H16), 2.03-2.05 (m, 1H, Hb-6), $3.32\left(\mathrm{td}, J^{1,3}=10,6 \mathrm{~Hz}, J^{1,2}=4,4 \mathrm{~Hz}, 1 \mathrm{H}, \mathrm{H1}\right)$, 4.32-4.34 (m, 2H, H15), 5.63 and 5.88 (d, $J=10,7 \mathrm{~Hz}, 2 \mathrm{H}, \mathrm{AB}$ system, H11), 7.23 (s, 1H, H12), 7.37 (s, 1H, H13), 7.51-7.52 (m, 2H, H27 and H28), 7.72-7.75 (m, 2H, H29 and H30), 10.40 (s, 1H, H14); ${ }^{13} \mathrm{C}$ NMR (300 MHz, $\mathrm{CDCl}_{3}, 298 \mathrm{~K}$ ) 8/ppm 13.91 (C20), 15.53 (C9 or C10), 20.94 (C7), 22.02 (C9 or C10), 22.35 (C19), 22.79 (C3), 25.47 (C8), 25.83 (C18), 30.18 (C17), 31.04 (C16), 31.06 (C5), 34.06 (C4), 40.22 (C6), 47.71 (C2), 50.29 (C15), 76.85 (C1), 79.74 (C11), 119.77 (C29), 121.15 (C12), 121.89 (C13), 123.12 (C30), 131.38 (C27), 131.84 (C28), 134.81 (C14), 138.11 (C31), 144.73 (C32), 170.50 (C33). Elemental analysis: calc. (\%) for $\mathrm{C}_{27} \mathrm{H}_{41} \mathrm{O}_{4} \mathrm{~N}_{3} \mathrm{~S}$ (503.70): C 64.38, H 8.20, N 8.34, found: C 64.49, H 8.38, N 8.19.

\section{3-Heptyl-1-[(1R,2S,5R)-(-)-menthoxymethyl]imidazolium saccharinate $\left[\mathrm{C}_{7}\right.$-Im- $\mathrm{CH}_{2} \mathrm{O}$ -}

Men][Sacc] (3g): Yield: 99.0\%, $0.0396 \mathrm{~mol}, 20.502 \mathrm{~g} .{ }^{1} \mathrm{H}$ NMR (600 MHz, $\left.\mathrm{CDCl}_{3}, 298 \mathrm{~K}\right) \delta / \mathrm{ppm}$ 0.49 (d, $J=7.0 \mathrm{~Hz}, 3 \mathrm{H}, \mathrm{H} 9$ or H10), 0.73-0.90 (m, 12H, Ha-4, H7, H9 or H10, Ha-6, Ha-3 and H21), 1.15-1.27 (m, 10H, H2, H5, H17, H18, H19 and H20), 1.52-1.56 (m, 2H, Hb-3, Hb-4), 1.831.89 (m, 3H, H8 and H16), 2.03-2.05 (m, 1H, Hb-6), $3.32\left(\mathrm{td}, J^{1,3}=10,6 \mathrm{~Hz}, J^{1,2}=4,4 \mathrm{~Hz}, 1 \mathrm{H}\right.$, H1), 4.30-4.33 (m, 2H, H15), 5.63 and 5.87 (d, $J=10,6 \mathrm{~Hz}, J=10,7 \mathrm{~Hz}, 2 \mathrm{H}, \mathrm{AB}$ system, H11), 7.24 (s, 1H, H12), 7.375 (s, 1H, H13), 7.50-7.53 (m, 2H, H27 and H28), 7.72-7.75 (m, 2H, H29 and $\mathrm{H} 30), 10.38$ (s, 1H, H14); ${ }^{13} \mathrm{C}$ NMR (300 MHz, $\mathrm{CDCl}_{3}, 298 \mathrm{~K}$ ) 8/ppm 14.01 (C21), 15.54 (C9 or C10), 20.94 (C7), 22.03 (C9 or C10), 22.48 (C20), 22.79 (C3), 25.46 (C8), 26.13 (C19), 28.60 (C18), 30.23 (C17), 31.06 (C5), 31.49 (C16), 34.06 (C4), 40.20 (C6), 47.70 (C2), 50.29 (C15), 76.86 (C1), 79.73 (C11), 119.78 (C29), 121.14 (C12), 121.90 (C13), 123.12 (C30), 131.30 (C27), 131.85 (C28), 134.77 (C14), 138.12 (C31), 144.71 (C32), 170.49 (C33). Elemental analysis: calc. (\%) for $\mathrm{C}_{28} \mathrm{H}_{43} \mathrm{O}_{4} \mathrm{~N}_{3} \mathrm{~S}$ (517.72): C 64.96, H 8.37, N 8.12, found: C 64.83, H 8.52, N 8.04. 
1-[(1R,2S,5R)-(-)-Menthoxymethyl]-3-oktylimidazolium saccharinate $\left[\mathrm{C}_{8}\right.$-Im-CH2O-

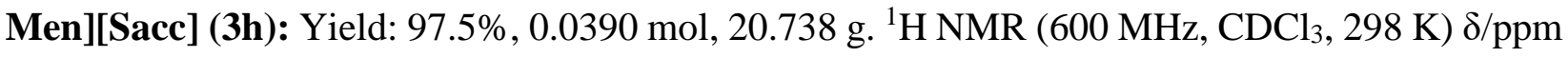
0.48 (d, $J=7.0 \mathrm{~Hz}, 3 \mathrm{H}, \mathrm{H} 9$ or H10), 0.77-0.94 (m, 12H, Ha-4, H7, H9 or H10, Ha-6, Ha-3 and H22), 1.19-1.34 (m, 12H, H2, H5, H17, H18, H19, H20 and H21), 1.56-1.60 (m, 2H, Hb-3, Hb-4), 1.87-1.93 (m, 3H, H8 and H16), 2.08-2.12 (m, 1H, Hb-6), $3.36\left(\mathrm{td}, J^{1,3}=10,5 \mathrm{~Hz}, J^{1,2}=4,2 \mathrm{~Hz}\right.$, 1H, H1), 4.34-4.37 (m, 2H, H15), 5.67 and 5.92 (d, J = 10,7 Hz, 2H, AB system, H11), 7.275 (s, 1H, H12), 7.41 (s, 1H, H13), 7.54-7.56 (m, 2H, H27 and H28), 7.75-7.79 (m, 2H, H29 and H30), 10.47 (s, 1H, H14); ${ }^{13} \mathrm{C}$ NMR (300 MHz, $\mathrm{CDCl}_{3}, 298 \mathrm{~K}$ ) $\delta / \mathrm{ppm} 14.04$ (C22), 15.55 (C9 or C10), 20.94 (C7), 22.03 (C9 or C10), 22.57 (C21), 22.80 (C3), 25.48 (C8), 26.19 (C20), 28.90 (C19), 29.00 (C18), 30.23 (C17), 31.06 (C5), 31.65 (C16), 34.06 (C4), 40.22 (C6), 47.72 (C2), 50.30 (C15), 76.85 (C1), 79.77 (C11), 119.78 (C29), 121.12 (C12), 121.85 (C13), 123.12 (C30), 131.28 (C27), 131.83 (C28), 134.80 (C14), 138.18 (C31), 144.73 (C32), 170.50 (C33). Elemental analysis: calc. (\%) for $\mathrm{C}_{29} \mathrm{H}_{45} \mathrm{O}_{4} \mathrm{~N}_{3} \mathrm{~S}$ (531.75): C 65.50, H 8.53, N 7.90, found: C 65.59, H 8.69, N 7.75.

1-[(1R,2S,5R)-(-)-Menthoxymethyl]-3-nonylimidazolium saccharinate [C9-Im-CH2OMen][Sacc] (3i): Yield: 97.0\%, $0.0388 \mathrm{~mol}, 21.176$ g. ${ }^{1} \mathrm{H}$ NMR (600 MHz, $\left.\mathrm{CDCl}_{3}, 298 \mathrm{~K}\right) \delta / \mathrm{ppm}$ 0.47 (d, $J=7.0 \mathrm{~Hz}, 3 \mathrm{H}, \mathrm{H} 9$ or H10), 0.75-0.94 (m, 12H, Ha-4, H7, H9 or H10, Ha-6, Ha-3 and H23), 1.19-1.32 (m, 14H, H2, H5, H17, H18, H19, H20, H21 and H22), 1.56-1.605 (m, 2H, Hb-3, Hb-4), 1.86-1.95 (m, 3H, H8 and H16), 2.07-2.09 (m, 1H, Hb-6), 3.36 (td, $J^{1,3}=10,6 \mathrm{~Hz}, J^{1,2}=4,3$ $\mathrm{Hz}, 1 \mathrm{H}, \mathrm{H1}$ ), 4.33-4.38 (m, 2H, H15), 5.67 and 5.92 (d, $J=10,6 \mathrm{~Hz}, J=10,7 \mathrm{~Hz}, 2 \mathrm{H}$, AB system, H11), 7.30 (s, 1H, H12), 7.42 (s, 1H, H13), 7.53-7.56 (m, 2H, H27 and H28), 7.75-7.79 (m, 2H,

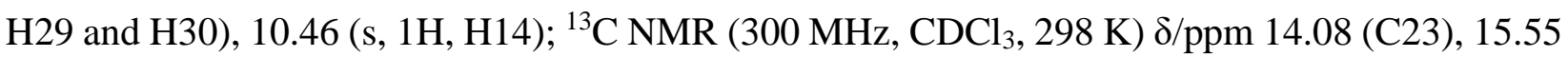
(C9 or C10), 20.93 (C7), 22.03 (C9 or C10), 22.61 (C22), 22.80 (C3), 25.46 (C8), 26.19 (C21), 28.95 (C20), 29.13 (C19), 29.30 (C18), 30.23 (C17), 31.06 (C5), 31.77 (C16), 34.06 (C4), 40.22 (C6), 47.70 (C2), 50.28 (C15), 76.86 (C1), 79.74 (C11), 119.76 (C29), 121.17 (C12), 121.91 (C13), 123.13 (C30), 131.28 (C27), 131.84 (C28), 134.78 (C14), 138.07 (C31), 144.72 (C32), 170.48 (C33). Elemental analysis: calc. (\%) for $\mathrm{C}_{30} \mathrm{H}_{47} \mathrm{O}_{4} \mathrm{~N}_{3} \mathrm{~S}$ (545.78): C 66.02, H 8.68, N 7.70, found: C 66.19, H 8.81, N 7.58. 
3-Decyl-1-[(1R,2S,5R)-(-)-menthoxymethyl]imidazolium saccharinate $\left[\mathrm{C}_{10}-\mathrm{Im}-\mathrm{CH}_{2} \mathrm{O}\right.$ Men][Sacc] (3j): Yield: 96.0\%, $0.0384 \mathrm{~mol}, 21.496 \mathrm{~g} .{ }^{1} \mathrm{H}$ NMR (600 MHz, $\mathrm{CDCl}_{3}, 298 \mathrm{~K}$ ) $\delta / \mathrm{ppm}$ 0.47 (d, $J=7.0 \mathrm{~Hz}, 3 \mathrm{H}, \mathrm{H} 9$ or H10), 0.77-0.92 (m, 12H, Ha-4, H7, H9 or H10, Ha-6, Ha-3 and H24), 1.19-1.33 (m, 16H, H2, H5, H17, H18, H19, H20, H21, H22 and H23), 1.56-1.60 (m, 2H, Hb-3, Hb-4), 1.88-1.93 (m, 3H, H8 and H16), 2.08-2.09 (m, 1H, Hb-6), 3.36 (td, $J^{1,3}=10,6 \mathrm{~Hz}$, $\left.J^{1,2}=4,3 \mathrm{~Hz}, 1 \mathrm{H}, \mathrm{H1}\right), 4.33-4.38(\mathrm{~m}, 2 \mathrm{H}, \mathrm{H} 15), 5.67$ and $5.92(\mathrm{~d}, J=10,6 \mathrm{~Hz}, J=10,7 \mathrm{~Hz}, 2 \mathrm{H}, \mathrm{AB}$ system, H11), 7.27 (S, 1H, H12), 7.41 (s, 1H, H13), 7.54-7.55 (m, 2H, H27 and H28), 7.75-7.77 (m, 2H, H29 and H30), 10.46 (s, 1H, H14); ${ }^{13} \mathrm{C}$ NMR (300 MHz, $\mathrm{CDCl}_{3}, 298 \mathrm{~K}$ ) $\delta / \mathrm{ppm} 14.11$ (C24), 15.55 (C9 or C10), 20.94 (C7), 22.02 (C9 or C10), 22.66 (C23), 22.80 (C3), 25.48 (C8), 26.20 (C22), 28.96 (C21), 29.24 (C20), 29.35 (C19), 29.44 (C18), 30.24 (C17), 31.06 (C5), 31.83 (C16), 34.06 (C4), 40.21 (C6), 47.72 (C2), 50.31 (C15), 76.84 (C1), 79.77 (C11), 119.78 (C29), 121.12 (C12), 121.84 (C13), 123.12 (C30), 131.28 (C27), 131.83 (C28), 134.81 (C14), 138.19 (C31), 144.74 (C32), 170.50 (C33). Elemental analysis: calc. (\%) for $\mathrm{C}_{31} \mathrm{H}_{49} \mathrm{O}_{4} \mathrm{~N}_{3} \mathrm{~S}$ (559.80): C 66.51, H 8.82, N 7.51, found: C 66.42, H 8.99, N 7.61.

1-[(1R,2S,5R)-(-)-Menthoxymethyl]-3-undecylimidazolium saccharinate [ $\mathrm{C}_{11}-\mathrm{Im}-\mathrm{CH}_{2} \mathrm{O}$ Men][Sacc] (3k): Yield: 97.5\%, $0.0390 \mathrm{~mol}, 22.379$ g. ${ }^{1} \mathrm{H}$ NMR (600 MHz, $\mathrm{CDCl}_{3}, 298 \mathrm{~K}$ ) $\delta / \mathrm{ppm}$ 0.485 (d, $J=7.2 \mathrm{~Hz}, 3 \mathrm{H}, \mathrm{H} 9$ or H10), 0.78-0.95 (m, 12H, Ha-4, H7, H9 or H10, Ha-6, Ha-3 and H25), 1.20-1.35 (m, 18H, H2, H5, H17, H18, H19, H20, H21, H22, H23 and H24), 1.57-1.62 (m, 2H, Hb-3, Hb-4), 1.89-1.94 (m, 3H, H8 and H16), 2.10-2.12 (m, 1H, Hb-6), 3.37-3.38 (m, 1H, H1), 4.36-4.38 (m, 2H, H15), 5.685 and 5.95 (d, $J=10,8 \mathrm{~Hz}, J=10,2 \mathrm{~Hz}, 2 \mathrm{H}$, AB system, H11), 7.29 (s, 1H, H12), 7.42 (t, $J=1,8 \mathrm{~Hz}, J=1,2 \mathrm{~Hz}, 1 \mathrm{H}, \mathrm{H} 13), 7.56-7.58$ (m, 2H, H27 and H28), 7.77-7.79

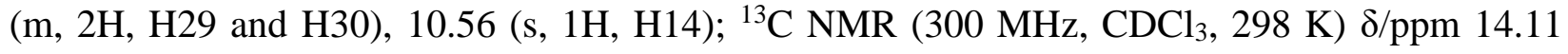
(C25), 15.55 (C9 or C10), 20.94 (C7), 22.02 (C9 or C10), 22.66 (C24), 22.79 (C3), 25.46 (C8), 26.20 (C23), 28.96 (C22), 29.24 (C21), 29.35 (C20), 29.46 (C19), 29.49 (C18), 30.25 (C17), 31.06 (C5), 31.83 (C16), 34.06 (C4), 40.21 (C6), 47.70 (C2), 50.30 (C15), 77.05 (C1), 79.72 (C11), 119.78 (C29), 121.12 (C12), 121.86 (C13), 123.12 (C30), 131.28 (C27), 131.84 (C28), 134.81 (C14), 138.19 (C31), 144.72 (C32), 170.50 (C33). Elemental analysis: calc. (\%) for $\mathrm{C}_{32} \mathrm{H}_{51} \mathrm{O}_{4} \mathrm{~N}_{3} \mathrm{~S}$ (573.83): C 66.98, H 8.96, N 7.32, found: C 66.77, H 9.11, N 7.49. 
3-Dodecyl-1-[(1R,2S,5R)-(-)-menthoxymethyl]imidazolium saccharinate [ $\mathrm{C}_{12}-\mathrm{Im}-\mathrm{CH}_{2} \mathrm{O}$ Men][Sacc] (3l): Yield: 96.5\%, $0.0386 \mathrm{~mol}, 22.691 \mathrm{~g} .{ }^{1} \mathrm{H}$ NMR (600 MHz, $\left.\mathrm{CDCl}_{3}, 298 \mathrm{~K}\right) \delta / \mathrm{ppm}$ 0.48 (d, $J=7.2 \mathrm{~Hz}, 3 \mathrm{H}, \mathrm{H} 9$ or H10), 0.78-0.94 (m, 12H, Ha-4, H7, H9 or H10, Ha-6, Ha-3 and H26), 1.20-1.34 (m, 20H, H2, H5, H17, H18, H19, H20, H21, H22, H23, H24 and H25), 1.57-1.61 (m, 2H, Hb-3, Hb-4), 1.88-1.93 (m, 3H, H8 and H16), 2.08-2.10 (m, 1H, Hb-6), 3.36-3.37 (m, 1H, H1), 4.34-4.38 (m, 2H, H15), 5.68 and 5.93 (d, $J=10,8$ Hz, 2H, AB system, H11), 7.31 (t, $J=1,2$ $\mathrm{Hz}, J=1,8 \mathrm{~Hz}, 1 \mathrm{H}, \mathrm{H} 12), 7.43$ (t, $J=1,8 \mathrm{~Hz}, 1 \mathrm{H}, \mathrm{H} 13), 7.55-7.565$ (m, 2H, H27 and H28), 7.767.765 (m, 2H, H29 and H30), 10.52 (s, 1H, H14); $\left.{ }^{13} \mathrm{C} \mathrm{NMR} \mathrm{(300} \mathrm{MHz,} \mathrm{CDCl}_{3}, 298 \mathrm{~K}\right) \delta / \mathrm{ppm}$ 14.11 (C26), 15.54 (C9 or C10), 20.94 (C7), 22.03 (C9 or C10), 22.66 (C25), 22.79 (C3), 25.46 (C8), 26.20 (C24), 28.96 (C23), 29.24 (C22), 29.35 (C21), 29.43 (C20), 29.49 (C19), 29.58 (C18), 30.25 (C17), 31.06 (C5), 31.83 (C16), 34.05 (C4), 40.22 (C6), 47.70 (C2), 50.28 (C15), 77.14 (C1), 79.73 (C11), 119.76 (C29), 121.16 (C12), 121.92 (C13), 123.12 (C30), 131.30 (C27), 131.84 (C28), 134.76 (C14), 138.13 (C31), 144.71 (C32), 170.46 (C33). Elemental analysis: calc. (\%) for $\mathrm{C}_{33} \mathrm{H}_{53} \mathrm{O}_{4} \mathrm{~N}_{3} \mathrm{~S}$ (587.86): C 67.42, H 9.09, N 7.15, found: C 67.59, H 9.25, N 7.02. 
Table S1. Analysis of the ionic impurities in the studied chiral ionic liquids [ $\left.\mathrm{Cn}_{n}-\mathrm{Im}-\mathrm{CH}_{2} \mathrm{OMen}\right][\mathrm{Sacc}](3 \mathrm{a}-3 \mathrm{I})$

\begin{tabular}{|c|c|c|c|c|c|c|c|c|c|c|c|c|c|}
\hline $\begin{array}{l}\text { Ionic } \\
\text { liquid }\end{array}$ & $\begin{array}{l}\mathrm{F}^{-} \\
(\mathrm{ppm})\end{array}$ & $\begin{array}{l}\mathrm{Cl}^{-} \\
(\mathrm{ppm})\end{array}$ & $\begin{array}{l}\mathrm{NO}_{2}^{-} \\
(\mathrm{ppm})\end{array}$ & $\begin{array}{l}\mathrm{SO}_{4}^{2-} \\
(\mathrm{ppm})\end{array}$ & $\begin{array}{l}\mathrm{Br}^{-} \\
(\mathrm{ppm})\end{array}$ & $\begin{array}{l}\mathrm{NO}_{3}^{-} \\
(\mathrm{ppm})\end{array}$ & $\begin{array}{l}\mathrm{H}_{2} \mathrm{PO}_{4}^{-} \\
(\mathrm{ppm})\end{array}$ & $\begin{array}{l}\mathrm{Na}^{+} \\
(\mathrm{ppm})\end{array}$ & $\begin{array}{l}\mathrm{NH}_{4}{ }^{+} \\
(\mathrm{ppm})\end{array}$ & $\begin{array}{l}\mathrm{K}^{+} \\
(\mathrm{ppm})\end{array}$ & $\begin{array}{l}\mathrm{Mg}^{2+} \\
(\mathrm{ppm})\end{array}$ & $\begin{array}{l}\mathrm{Ca}^{2+} \\
(\mathrm{ppm})\end{array}$ & $\begin{array}{l}\mathrm{Li}^{+} \\
(\mathrm{ppm})\end{array}$ \\
\hline 3a & n.a. & 0.021 & 0.037 & 0.013 & 0.005 & 0.019 & n.a. & 0.014 & 0.001 & 0.007 & 0.000 & 0.010 & 0.00002 \\
\hline $3 b$ & n.a. & 0.456 & 0.062 & 0.032 & 0.013 & 0.033 & n.a. & 0.043 & 0.001 & 0.012 & 0.002 & 0.020 & 0.00003 \\
\hline $3 c$ & n.a. & n.a. & n.a. & 0.013 & n.a. & 0.037 & n.a. & 0.018 & 0.000 & 0.010 & 0.000 & 0.013 & 0.00002 \\
\hline $3 d$ & n.a. & 0.014 & 0.029 & 0.011 & 0.010 & 0.041 & n.a. & 0.007 & 0.003 & 0.026 & 0.000 & 0.013 & n.a. \\
\hline $3 e$ & n.a. & n.a. & n.a. & n.a. & n.a. & 0.076 & n.a. & 0.018 & 0.001 & 0.011 & 0.000 & 0.017 & 0.00003 \\
\hline $3 f$ & n.a. & 0.014 & n.a. & n.a. & n.a. & 0.012 & n.a. & 0.003 & 0.007 & 0.004 & 0.000 & 0.007 & n.a. \\
\hline $3 g$ & n.a. & 0.010 & n.a. & n.a. & n.a. & 0.017 & n.a. & 0.004 & 0.005 & 0.005 & 0.000 & 0.007 & n.a. \\
\hline $3 h$ & n.a. & 0.050 & 0.044 & 0.005 & n.a. & 0.014 & n.a. & 0.003 & 0.004 & 0.003 & 0.000 & 0.005 & n.a. \\
\hline $3 \mathbf{i}$ & n.a. & 0.336 & 0.066 & 0.016 & 0.006 & n.a. & n.a. & 0.005 & 0.003 & 0.008 & 0.000 & 0.010 & n.a. \\
\hline $3 \mathbf{j}$ & n.a. & 0.010 & 0.026 & 0.008 & 0.003 & n.a. & n.a. & 0.006 & 0.003 & 0.006 & 0.000 & 0.006 & n.a. \\
\hline $3 \mathbf{k}$ & n.a. & 0.271 & 0.027 & 0.008 & 0.003 & 0.020 & n.a. & 0.005 & 0.004 & 0.006 & 0.000 & 0.006 & n.a. \\
\hline 31 & n.a. & 0.129 & 0.045 & 0.012 & 0.008 & 0.039 & n.a. & 0.006 & 0.008 & 0.008 & n.a. & 0.010 & n.a. \\
\hline
\end{tabular}


Table S2. Abbreviation, yield, cationic active substance content, empirical formula, elementary analysis calculation and found for the precursors, 3-alkyl-1-[(1R,2S,5R)-(-)-menthoxymethyl]imidazolium chlorides (2a-2l)

\begin{tabular}{|c|c|c|c|c|c|c|c|}
\hline CILs & $\begin{array}{l}\text { Abbreviation } \\
{\left[\mathrm{C}_{\mathrm{n}}-\mathrm{Im}-\mathrm{CH}_{2} \mathrm{OMen}\right][\mathrm{Cl}]}\end{array}$ & $\mathrm{R}$ & $\begin{array}{l}\text { Yield }^{a, b} \\
{[\%]}\end{array}$ & $\begin{array}{l}\text { Surfactant } \\
\text { content }^{c} \\
{[\%]}\end{array}$ & $\begin{array}{l}\text { Empirical } \\
\text { formula }\end{array}$ & $\begin{array}{l}\text { Elementary analysis } \\
\text { calculation } \\
\text { [\%] }\end{array}$ & $\begin{array}{l}\text { Elementary analysis } \\
\text { found } \\
{[\%]}\end{array}$ \\
\hline $2 \mathbf{a}$ & {$\left[\mathrm{C}_{1}-\mathrm{Im}-\mathrm{CH}_{2} \mathrm{OMen}\right][\mathrm{Cl}]$} & $\mathrm{CH}_{3}$ & 99.5 & 99.9 & $\mathrm{C}_{15} \mathrm{H}_{27} \mathrm{OClN}_{2}$ & C 62.81, H 9.49, N 9.77 & C 62.94, H 9.63, N 9.59 \\
\hline $2 \mathbf{b}$ & {$\left[\mathrm{C}_{2}-\mathrm{Im}-\mathrm{CH}_{2} \mathrm{OMen}\right][\mathrm{Cl}]$} & $\mathrm{C}_{2} \mathrm{H}_{5}$ & 99.0 & 99.9 & $\mathrm{C}_{16} \mathrm{H}_{29} \mathrm{OClN}_{2}$ & С 63.87, H 9.71, N 9.31 & C 63.71, H 9.94, N 9.40 \\
\hline $2 c$ & {$\left[\mathrm{C}_{3}-\mathrm{Im}-\mathrm{CH}_{2} \mathrm{OMen}\right][\mathrm{Cl}]$} & $\mathrm{C}_{3} \mathrm{H}_{7}$ & 99.5 & 99.8 & $\mathrm{C}_{17} \mathrm{H}_{31} \mathrm{OClN}_{2}$ & C 64.84, H 9.92, N 8.90 & C 64.73, H 10.08, N 9.01 \\
\hline 2d & {$\left[\mathrm{C}_{4}-\mathrm{Im}-\mathrm{CH}_{2} \mathrm{OMen}\right][\mathrm{Cl}]$} & $\mathrm{C}_{4} \mathrm{H}_{9}$ & 99.5 & 99.9 & $\mathrm{C}_{18} \mathrm{H}_{33} \mathrm{OClN}_{2}$ & C 65.73, H 10.11, N 8.52 & C 65.89, H 10.32, N 8.39 \\
\hline $2 e$ & {$\left[\mathrm{C}_{5}-\mathrm{Im}-\mathrm{CH}_{2} \mathrm{OMen}\right][\mathrm{Cl}]$} & $\mathrm{C}_{5} \mathrm{H}_{11}$ & 99.0 & 99.7 & $\mathrm{C}_{19} \mathrm{H}_{35} \mathrm{OClN}_{2}$ & C 66.54, H 10.29, N 8.17 & C 66.71, H 10.41, N 8.03 \\
\hline $2 f$ & {$\left[\mathrm{C}_{6}-\mathrm{Im}-\mathrm{CH}_{2} \mathrm{OMen}\right][\mathrm{Cl}]$} & $\mathrm{C}_{6} \mathrm{H}_{13}$ & 98.5 & 99.7 & $\mathrm{C}_{20} \mathrm{H}_{37} \mathrm{OClN}_{2}$ & C 67.30, H 10.45, N 7.85 & C 67.17, H 10.64, N 7.95 \\
\hline $2 \mathrm{~g}$ & {$\left[\mathrm{C}_{7}-\mathrm{Im}-\mathrm{CH}_{2} \mathrm{OMen}\right][\mathrm{Cl}]$} & $\mathrm{C}_{7} \mathrm{H}_{15}$ & 98.0 & 99.8 & $\mathrm{C}_{21} \mathrm{H}_{39} \mathrm{OClN}_{2}$ & C 67.98, H 10.60, N 7.55 & C 67.77, H 10.89, N 7.62 \\
\hline $2 \mathrm{~h}$ & {$\left[\mathrm{C}_{8}-\mathrm{Im}-\mathrm{CH}_{2} \mathrm{OMen}\right][\mathrm{Cl}]$} & $\mathrm{C}_{8} \mathrm{H}_{17}$ & 98.0 & 99.7 & $\mathrm{C}_{22} \mathrm{H}_{41} \mathrm{OClN}_{2}$ & C 68.63, H 10.73, N 7.28 & C 68.81, H 10.92, N 7.17 \\
\hline $2 \mathbf{i}$ & {$\left[\mathrm{C}_{9}-\mathrm{Im}-\mathrm{CH}_{2} \mathrm{OMen}\right][\mathrm{Cl}]$} & $\mathrm{C}_{9} \mathrm{H}_{19}$ & 97.0 & 99.6 & $\mathrm{C}_{23} \mathrm{H}_{43} \mathrm{OClN}_{2}$ & C 69.225, H 10.86, N 7.02 & C 69.38, H 10.99, N 6.92 \\
\hline $2 \mathbf{j}$ & {$\left[\mathrm{C}_{10}-\mathrm{Im}-\mathrm{CH}_{2} \mathrm{OMen}\right][\mathrm{Cl}]$} & $\mathrm{C}_{10} \mathrm{H}_{21}$ & 97.5 & 99.6 & $\mathrm{C}_{24} \mathrm{H}_{45} \mathrm{OClN}_{2}$ & C 69.78, H 10.98, N 6.78 & C 69.56, H 11.12, N 6.66 \\
\hline $2 \mathrm{k}$ & {$\left[\mathrm{C}_{11}-\mathrm{Im}-\mathrm{CH}_{2} \mathrm{OMen}\right][\mathrm{Cl}]$} & $\mathrm{C}_{11} \mathrm{H}_{23}$ & 97.0 & 98.5 & $\mathrm{C}_{25} \mathrm{H}_{47} \mathrm{OClN}_{2}$ & C 70.30, H 11.09, N 6.56 & C 70.44, H 11.31, N 6.48 \\
\hline 21 & {$\left[\mathrm{C}_{12}-\mathrm{Im}-\mathrm{CH}_{2} \mathrm{OMen}\right][\mathrm{Cl}]$} & $\mathrm{C}_{12} \mathrm{H}_{25}$ & 97.5 & 98.3 & $\mathrm{C}_{26} \mathrm{H}_{49} \mathrm{OClN}_{2}$ & C 70.79, H 11.20, N 6.35 & C 70.99, H 11.45, N 6.27 \\
\hline
\end{tabular}


Table S3. Solubility of synthesized salts (3a-3I) at $25^{\circ} \mathrm{C}^{a}$ and $50^{\circ} \mathrm{C}^{b}$

\begin{tabular}{|c|c|c|c|c|c|c|c|c|c|c|c|c|c|c|c|}
\hline \multirow[b]{2}{*}{$\begin{array}{l}\text { Salt } \\
\text { no }\end{array}$} & \multicolumn{15}{|c|}{ Solvent } \\
\hline & Water & Methanol & $\begin{array}{l}1- \\
\text { Propanol }\end{array}$ & $\begin{array}{l}2- \\
\text { Propanol }\end{array}$ & DMSO & DMF & THF & Toluene & Acetonitrile & $\begin{array}{l}\text { Diethyl } \\
\text { ether }\end{array}$ & Hexane & Chloroform & $\begin{array}{l}\text { Methylene } \\
\text { chloride }\end{array}$ & $\begin{array}{l}\text { Ethyl } \\
\text { acetate }\end{array}$ & Acetone \\
\hline $3 a$ & $\mathrm{I}^{a . b}$ & $\mathrm{~S}$ & S & $\mathrm{S}$ & $\mathrm{S}$ & $\mathrm{S}$ & $\mathrm{S}$ & $S$ & $\mathrm{~S}$ & $\mathrm{I}^{a . b}$ & $\mathrm{I}^{a . b}$ & $\mathrm{~S}$ & S & S & $\mathrm{S}$ \\
\hline $3 b$ & $\mathrm{I}^{a . b}$ & S & S & $S$ & S & $\mathrm{S}$ & $S$ & $S$ & S & $\mathrm{I}^{a . b}$ & $\mathrm{I}^{a . b}$ & S & $S$ & $S$ & S \\
\hline $3 c$ & $\mathrm{I}^{a . b}$ & $S$ & $\mathrm{~S}$ & $\mathrm{~S}$ & $\mathrm{~S}$ & $\mathrm{~S}$ & $\mathrm{~S}$ & $S$ & $\mathrm{~S}$ & $\mathrm{I}^{a . b}$ & $\mathrm{I}^{a . b}$ & $\mathrm{~S}$ & $\mathrm{~S}$ & $\mathrm{~S}$ & $\mathrm{~S}$ \\
\hline 3d & $\mathrm{I}^{a . b}$ & S & $\mathrm{S}$ & $\mathrm{S}$ & $\mathrm{S}$ & $\mathrm{S}$ & $\mathrm{S}$ & $\mathrm{S}$ & S & $\mathrm{I}^{a . b}$ & $\mathrm{I}^{a . b}$ & $\mathrm{~S}$ & $\mathrm{~S}$ & $\mathrm{~S}$ & $\mathrm{~S}$ \\
\hline $3 \mathbf{e}$ & $\mathrm{I}^{a . b}$ & S & S & S & S & S & $S$ & S & S & $\mathrm{I}^{a . b}$ & $\mathrm{I}^{a . b}$ & $S$ & $S$ & S & S \\
\hline $3 f$ & $\mathrm{I}^{a . b}$ & S & S & S & $S$ & S & $S$ & S & $S$ & $\mathrm{I}^{a . b}$ & $\mathrm{I}^{a . b}$ & S & $S$ & $S$ & S \\
\hline $3 g$ & $\mathrm{I}^{a . b}$ & $S$ & S & S & S & S & S & S & S & $\mathrm{I}^{a . b}$ & $\mathrm{I}^{a . b}$ & S & S & S & S \\
\hline $3 \mathbf{h}$ & $\mathrm{I}^{a . b}$ & S & S & S & $S$ & S & $S$ & S & $S$ & $\mathrm{I}^{a . b}$ & $\mathrm{I}^{a . b}$ & $S$ & $S$ & $S$ & S \\
\hline $3 \mathbf{i}$ & $\mathrm{I}^{a . b}$ & S & S & S & $S$ & S & $S$ & S & $S$ & $\mathrm{I}^{a . b}$ & $\mathrm{I}^{a . b}$ & S & S & $S$ & S \\
\hline $3 \mathbf{j}$ & $\mathrm{I}^{a . b}$ & S & S & $\mathrm{S}$ & $S$ & S & $S$ & $S$ & $S$ & $\mathrm{I}^{a . b}$ & $\mathrm{I}^{a . b}$ & $S$ & $S$ & $S$ & S \\
\hline $3 \mathbf{k}$ & $\mathrm{I}^{a . b}$ & S & S & S & $S$ & S & $S$ & S & S & $\mathrm{I}^{a . b}$ & $\mathrm{I}^{a . b}$ & $S$ & S & $S$ & S \\
\hline 31 & $\mathrm{I}^{a . b}$ & $\mathrm{~S}$ & $\mathrm{~S}$ & $\mathrm{~S}$ & $\mathrm{~S}$ & $\mathrm{~S}$ & $S$ & $\mathrm{~S}$ & S & $\mathrm{I}^{a . b}$ & $\mathrm{I}^{a . b}$ & $\mathrm{~S}$ & $\mathrm{~S}$ & $\mathrm{~S}$ & $\mathrm{~S}$ \\
\hline
\end{tabular}

S - complete soluble. I - insoluble. DMSO - dimethyl sulfoxide. DMF - dimethylformamide. THF - tetrahydrofuran. 
Table S4. Density. $\rho\left(\mathrm{g} \cdot \mathrm{cm}^{-3}\right)$ of 3-alkyl-1-[(1R.2S.5R)-(-)-menthoxymethyl]imidazolium saccharinate ionic liquids. [C $\mathrm{C}_{\mathrm{n}}$-im$\left.\mathrm{CH}_{2} \mathrm{OMen}\right][\mathrm{Sacc}]$ ILs $(n=4-12)$ at $t=(30-65)^{\circ} \mathrm{C}$

\begin{tabular}{|c|c|c|c|c|c|c|c|c|c|}
\hline \multirow[b]{2}{*}{$t /{ }^{\circ} \mathrm{C}$} & \multicolumn{9}{|c|}{$\rho / \mathrm{g} \cdot \mathrm{cm}^{-3}$} \\
\hline & $\begin{array}{c}{\left[\mathrm{C}_{4} \text {-im- }\right.} \\
\left.\mathrm{CH}_{2} \mathrm{OMen}\right] \\
{[\mathrm{Sacc}]} \\
\end{array}$ & $\begin{array}{c}{\left[\mathrm{C}_{5} \text {-im- }\right.} \\
\left.\mathrm{CH}_{2} \mathrm{OMen}\right] \\
{[\mathrm{Sacc}]} \\
\end{array}$ & $\begin{array}{c}{\left[\mathrm{C}_{6}-\mathrm{im}-\right.} \\
\left.\mathrm{CH}_{2} \mathrm{OMen}\right] \\
{[\mathrm{Sacc}]} \\
\end{array}$ & $\begin{array}{c}{\left[\mathrm{C}_{7-\mathrm{im}-}\right.} \\
\left.\mathrm{CH}_{2} \mathrm{OMen}\right] \\
{[\mathrm{Sacc}]} \\
\end{array}$ & $\begin{array}{c}{\left[\mathrm{C}_{8} \text {-im- }\right.} \\
\left.\mathrm{CH}_{2} \mathrm{OMen}\right] \\
{[\mathrm{Sacc}]} \\
\end{array}$ & $\begin{array}{c}{\left[\mathrm{C}_{9}-\mathrm{im}-\right.} \\
\left.\mathrm{CH}_{2} \mathrm{OMen}\right] \\
{[\mathrm{Sacc}]} \\
\end{array}$ & $\begin{array}{c}{\left[\mathrm{C}_{10-\mathrm{im}-}\right.} \\
\left.\mathrm{CH}_{2} \mathrm{OMen}\right] \\
{[\mathrm{Sacc}]}\end{array}$ & $\begin{array}{c}{\left[\mathrm{C}_{11-\mathrm{im}-}\right.} \\
\left.\mathrm{CH}_{2} \mathrm{OMen}\right] \\
{[\mathrm{Sacc}]} \\
\end{array}$ & $\begin{array}{c}{\left[\mathrm{C}_{12} \text {-im- }\right.} \\
\left.\mathrm{CH}_{2} \mathrm{OMen}\right] \\
{[\text { Sacc }]} \\
\end{array}$ \\
\hline 30 & 1.1371 & 1.1213 & 1.1102 & 1.0984 & 1.0843 & $1.0758^{a}$ & 1.0727 & 1.0664 & 1.0597 \\
\hline 35 & 1.1339 & 1.1180 & 1.1068 & 1.0951 & 1.0809 & 1.0723 & 1.0694 & 1.0628 & 1.0559 \\
\hline 40 & 1.1305 & 1.1147 & 1.1035 & 1.0917 & 1.0774 & 1.0690 & 1.0661 & 1.0591 & 1.0516 \\
\hline 45 & 1.1272 & 1.1113 & 1.1000 & 1.0883 & 1.0738 & 1.0657 & 1.0627 & 1.0554 & 1.0471 \\
\hline 50 & 1.1238 & 1.1079 & 1.0963 & 1.0847 & 1.0701 & 1.0623 & 1.0592 & 1.0519 & 1.0433 \\
\hline 55 & 1.1202 & 1.1042 & 1.0929 & 1.0811 & 1.0666 & 1.0588 & 1.0556 & 1.0485 & 1.0395 \\
\hline 60 & 1.1167 & 1.1008 & 1.0896 & 1.0778 & 1.0630 & 1.0554 & 1.0524 & 1.0450 & 1.0355 \\
\hline 65 & 1.1135 & 1.0976 & 1.0863 & 1.0746 & 1.0589 & 1.0522 & 1.0492 & 1.0416 & 1.0312 \\
\hline
\end{tabular}


Table S5. Surface tension. $\gamma\left(\mathrm{mN} \cdot \mathrm{m}^{-1}\right)$ of 3-alkyl-1-[(1R.2S.5R)-(-)-menthoxymethyl]imidazolium saccharinate ionic liquids. $\left[\mathrm{C}_{\mathrm{n}}\right.$-im-CH2OMen] $\mathrm{CHacc}_{2} \mathrm{ILs}(n=4-12)$ at $t=(30-60)^{\circ} \mathrm{C}$

\begin{tabular}{|c|c|c|c|c|c|c|c|c|c|}
\hline$t /{ }^{\circ} \mathrm{C}$ & \multicolumn{9}{|c|}{$\gamma / \mathrm{mN} \cdot \mathrm{m}^{-1}$} \\
\hline 30 & 34.63 & 34.05 & 32.82 & 32.05 & 31.62 & 31.13 & 31.14 & 31.16 & 31.12 \\
\hline 35 & 34.36 & 33.77 & 32.56 & 31.84 & 31.39 & 30.86 & 30.88 & 30.90 & 30.84 \\
\hline 45 & 33.78 & 33.25 & 32.04 & 31.39 & 30.92 & 30.42 & 30.35 & 30.36 & 30.21 \\
\hline 50 & 33.55 & 32.98 & 31.75 & 31.14 & 30.68 & 30.21 & 30.08 & 30.09 & 29.93 \\
\hline 55 & 33.24 & 32.68 & 31.50 & 30.90 & 30.42 & 29.99 & 29.81 & 29.81 & 29.62 \\
\hline 60 & - & - & - & - & 30.19 & 29.74 & 29.58 & 29.56 & 29.23 \\
\hline
\end{tabular}




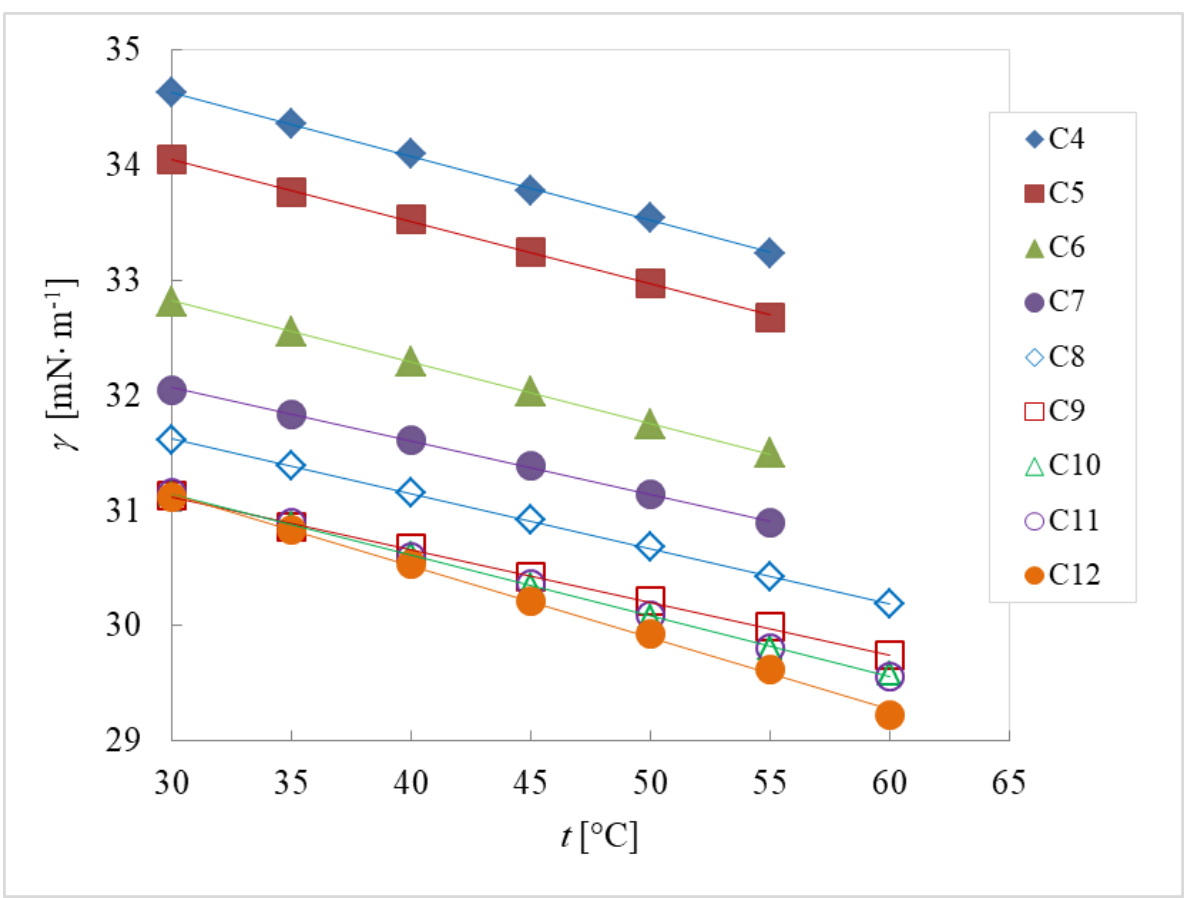

Figure S1. Surface tension of 3-alkyl-1-[(1R.2S.5R)-(-)-menthoxymethyl]imidazolium saccharinates at different temperatures. 


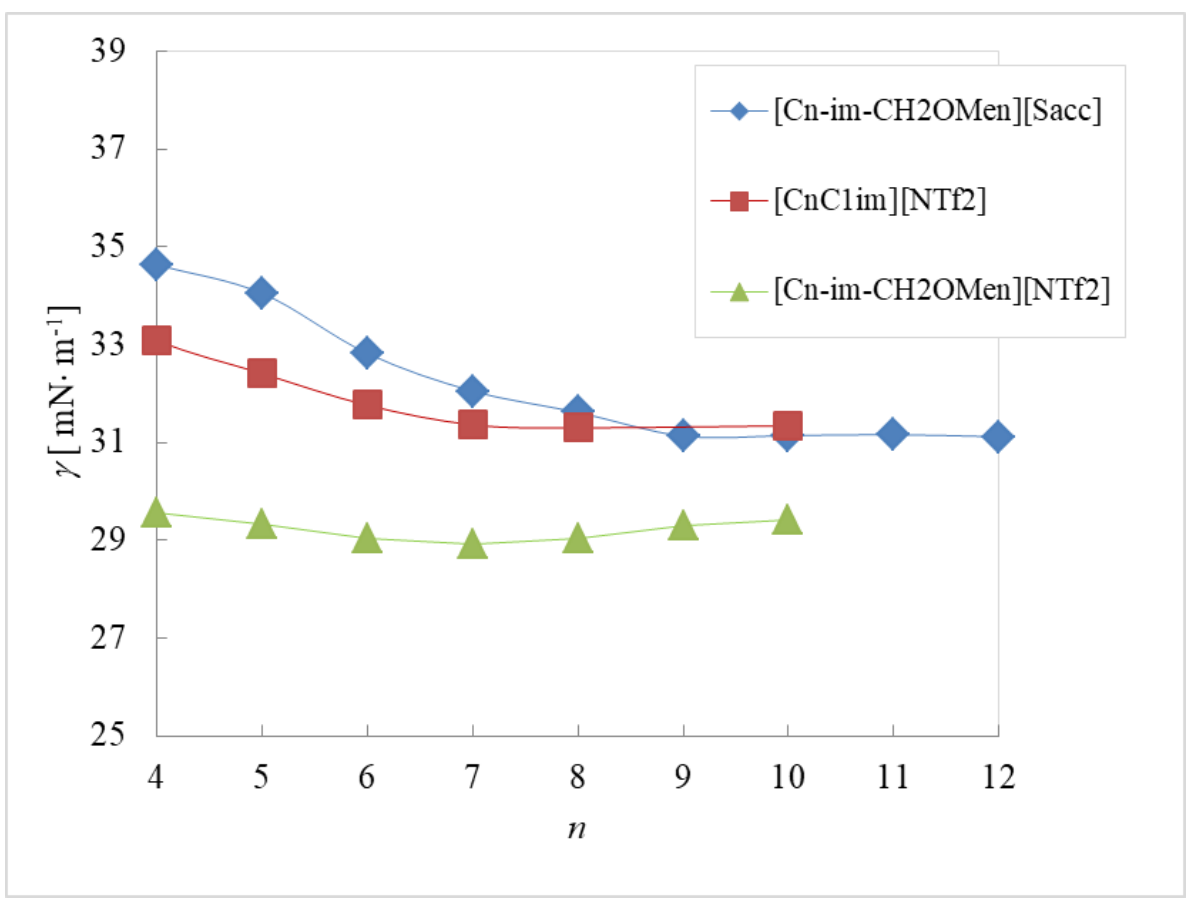

Figure S2. Surface tension at $30{ }^{\circ} \mathrm{C}$ of 3-alkyl-1-[(1R.2S.5R)-(-)-menthoxymethyl]imidazolium saccharinate ILs $\left(\left[\mathrm{C}_{\mathrm{n}}-\mathrm{im}-\mathrm{CH}_{2} \mathrm{OMen}\right][\mathrm{Sacc}]\right)$ compared with $\gamma$ of 1-alkyl-3-methylimidazolium bis(trifluoromethylsulfonyl)imide ILs $\left(\left[\mathrm{C}_{\mathrm{n}} \mathrm{C}_{1} \mathrm{im}\right]\left[\mathrm{NTf}_{2}\right]\right){ }^{7}$ and of 3-alkyl-1-[(1R.2S.5R)-(-)menthoxymethyl]imidazolium bis(trifluoromethylsulfonyl)imide ILs $\left(\left[\mathrm{C}_{\mathrm{n}}-\mathrm{im}-\mathrm{CH}_{2} \mathrm{OMen}\right]\left[\mathrm{NTf}_{2}\right]\right){ }^{8}$ 


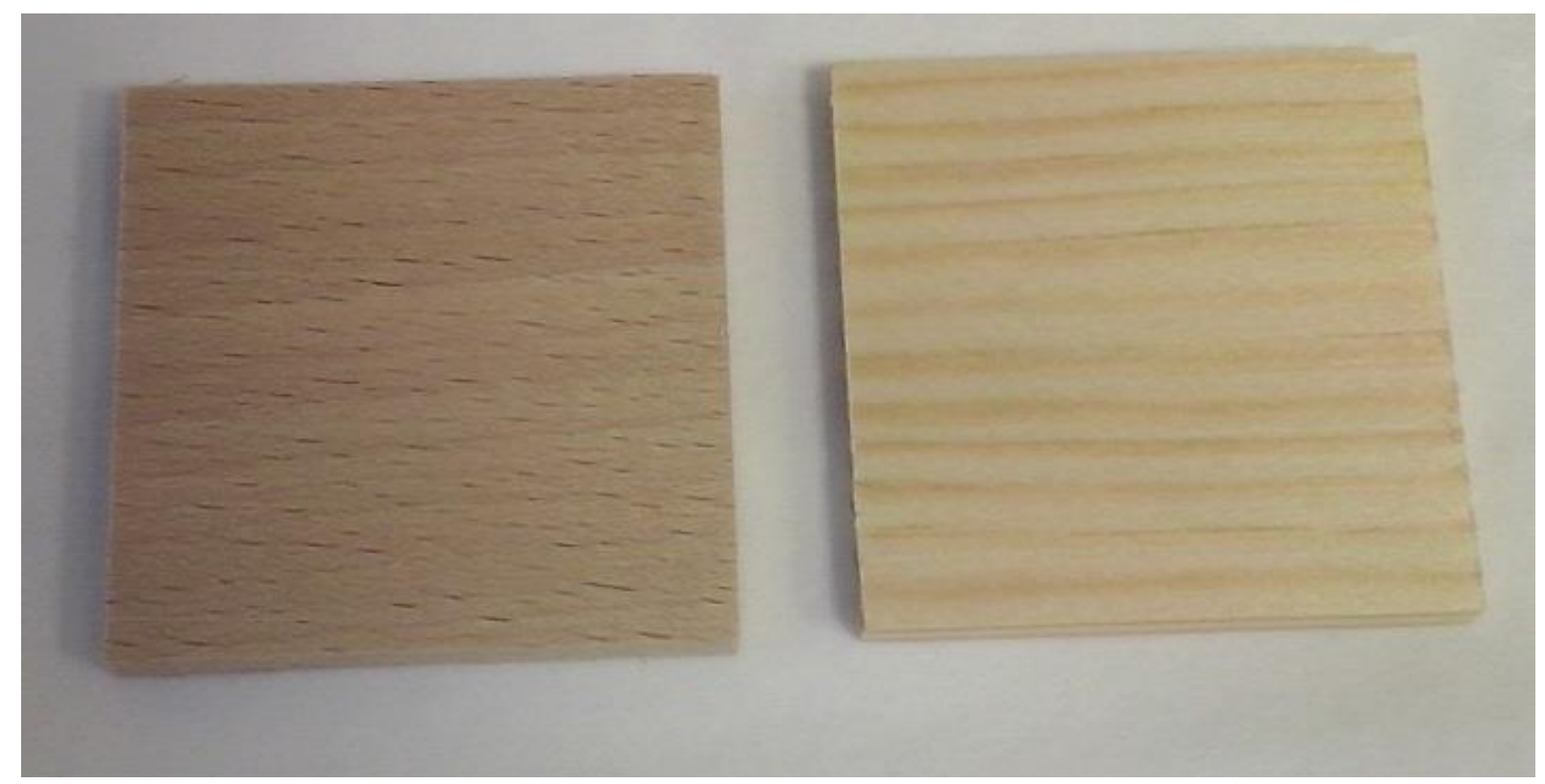

Figure S3a. Photos of beech wood (left) and Scots pine wood (right).

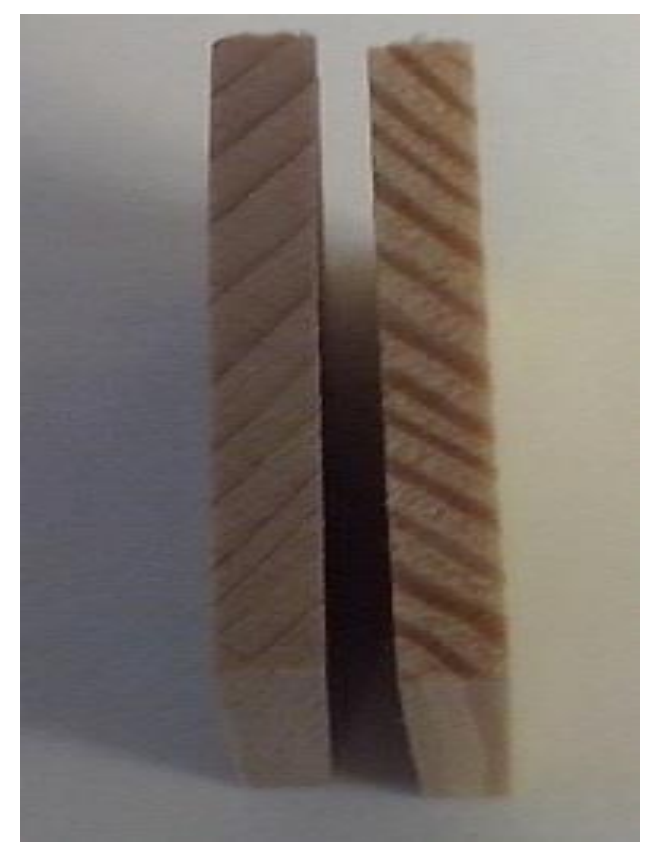

Figure S3b. Profiles of beech wood (left) and pine wood (right). 


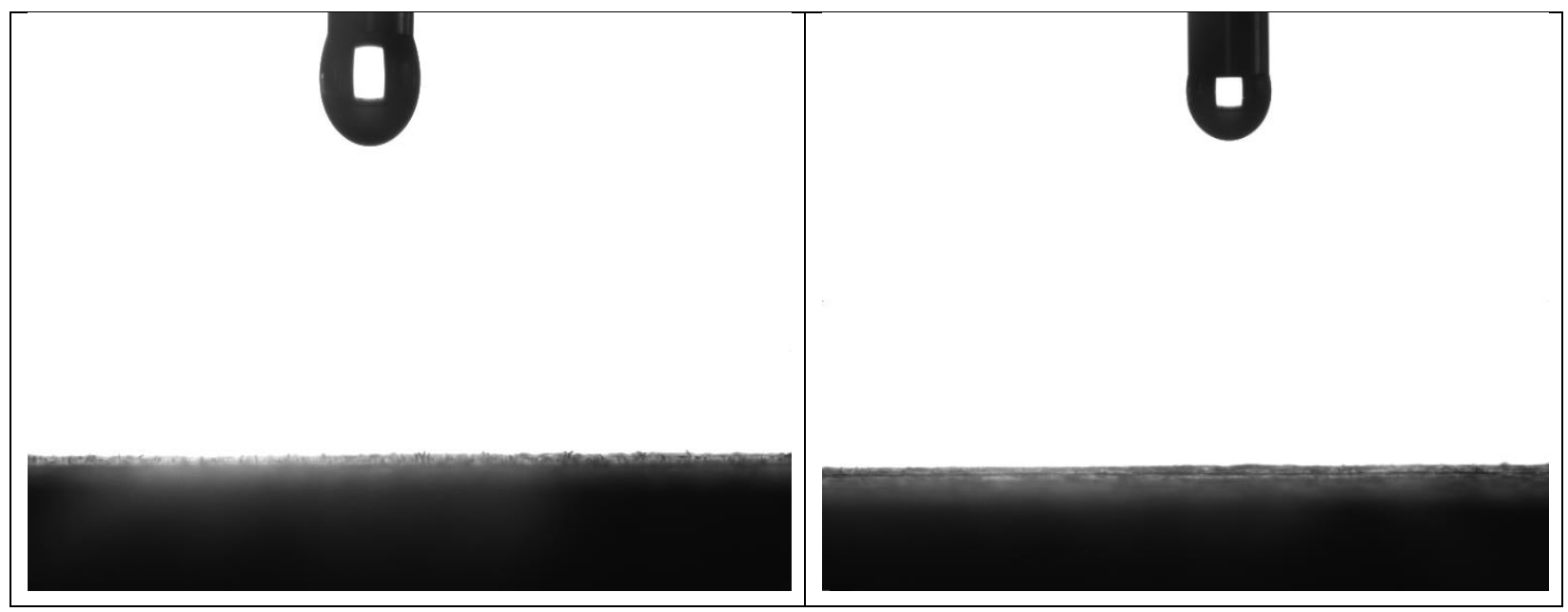

Figure S4. Photo of beech wood (left) and pine wood (right) before measurements.

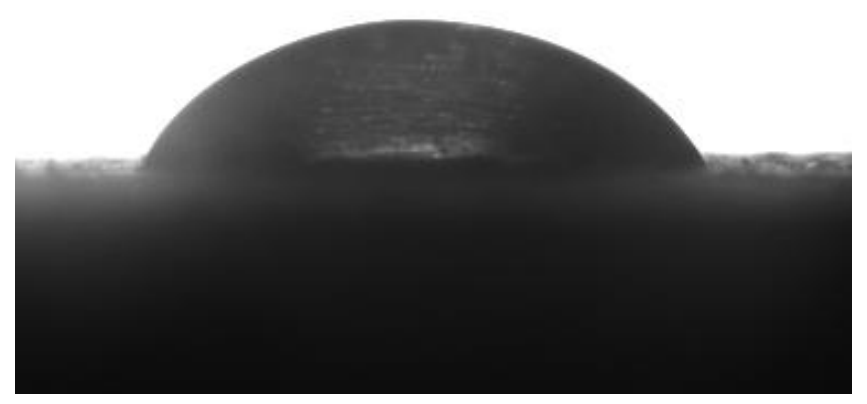

Figure S5. Photo of drop of $\mathrm{C}_{8}$ saccharinate at $30^{\circ} \mathrm{C}$ on the pine wood after 15 seconds.

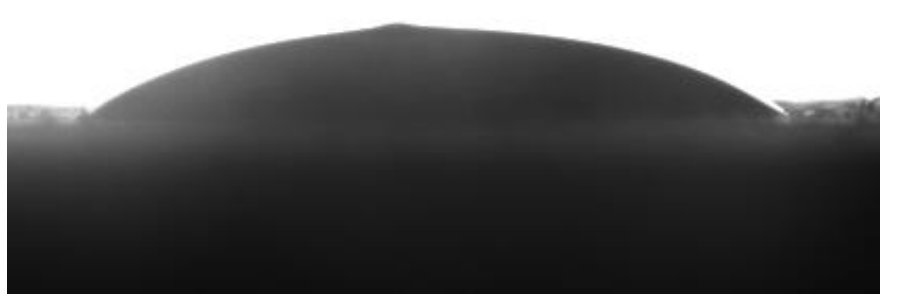

Figure S6. Photo of drop of $\mathrm{C}_{8}$ saccharinate $30^{\circ} \mathrm{C}$ on the pine wood after 2 minutes. 


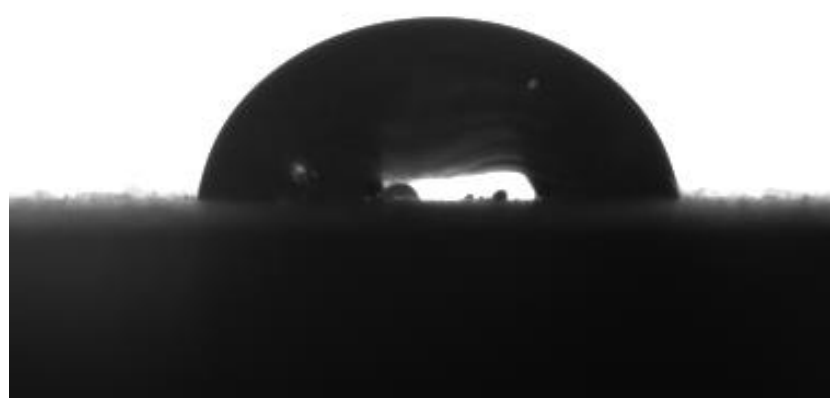

Figure S7. Photo of drop of $\mathrm{C}_{8}$ saccharinate at $30^{\circ} \mathrm{C} \mathrm{K}$ on the beech wood after 15 seconds.

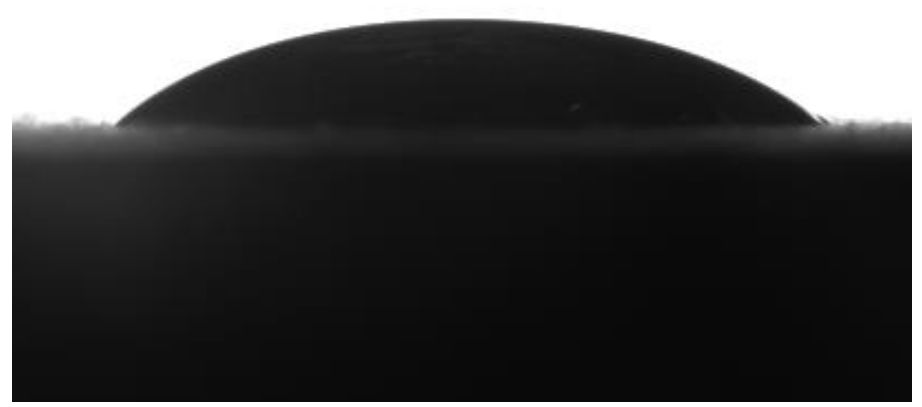

Figure S8. Photo of drop of $\mathrm{C}_{8}$ saccharinate at $30^{\circ} \mathrm{C}$ on the beech wood after 1 minute.

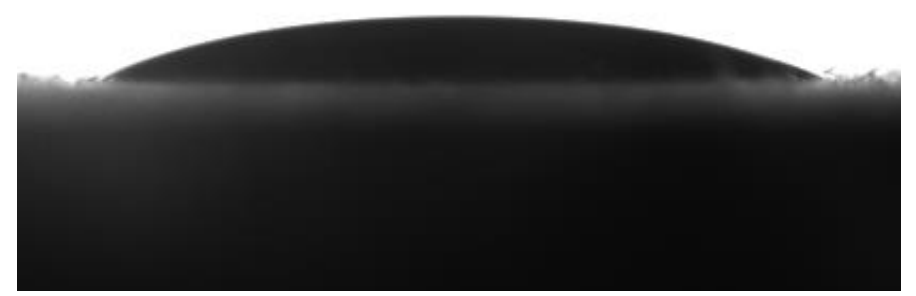

Figure S9. Photo of drop of $\mathrm{C}_{8}$ saccharinate at $30^{\circ} \mathrm{C}$ on the beech wood after 4 minutes. 


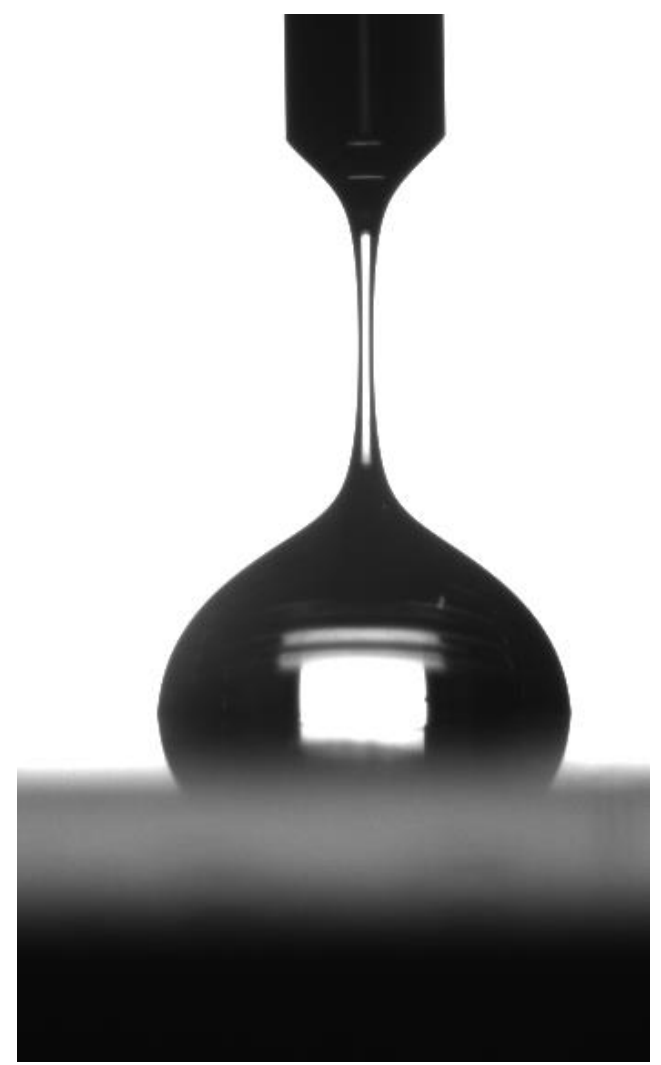

Figure S10. Photo of drop of $\mathrm{C}_{6}$ saccharinate at $30^{\circ} \mathrm{C} \mathrm{K}$ on glass during formation of the drop.
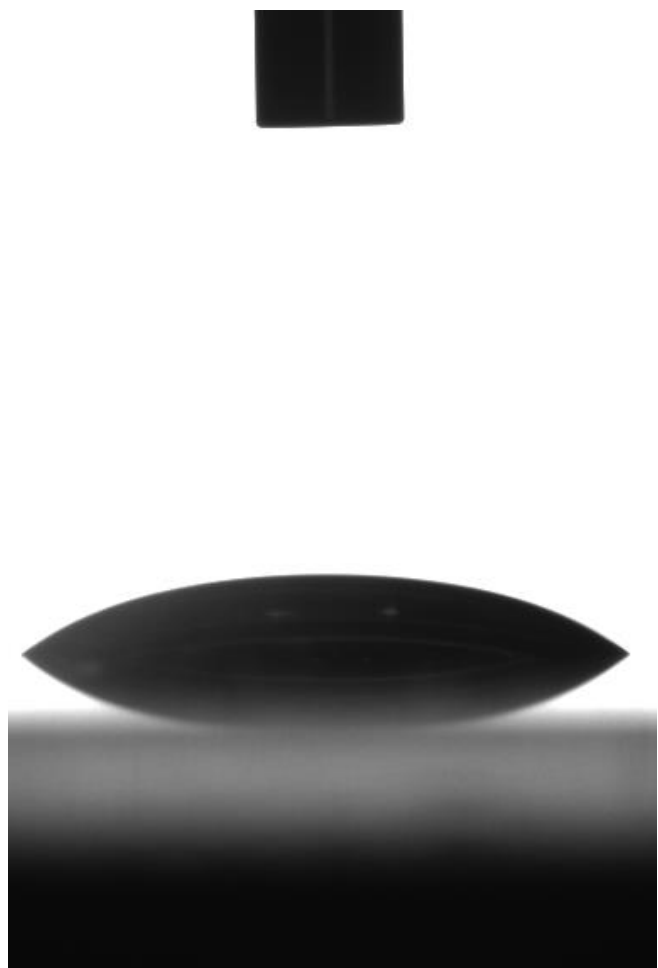

Figure S11. Photo of drop of $\mathrm{C}_{6}$ saccharinate at $303.2 \mathrm{~K}$ on glass after 4.5 minutes. 


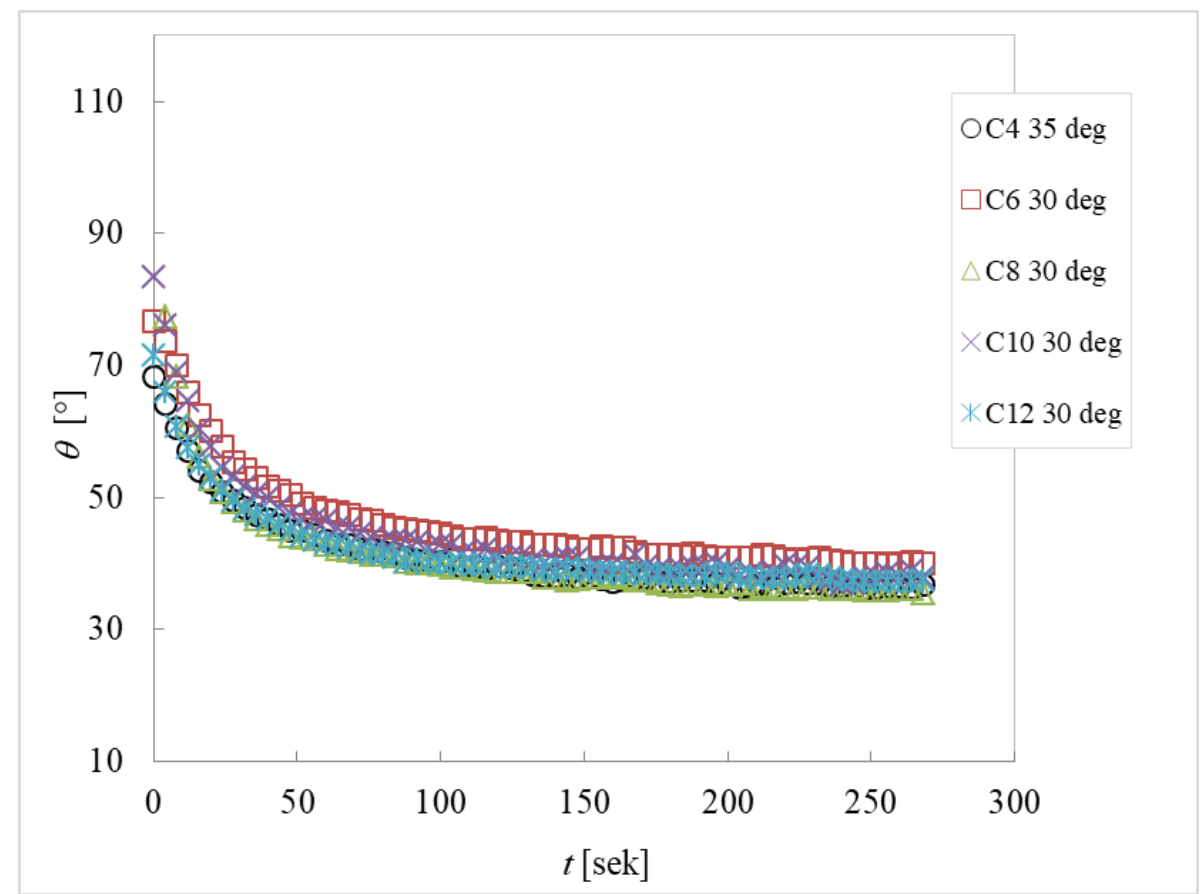

Figure S12. Contact angle of "sweet" ionic liquids on optic glass.

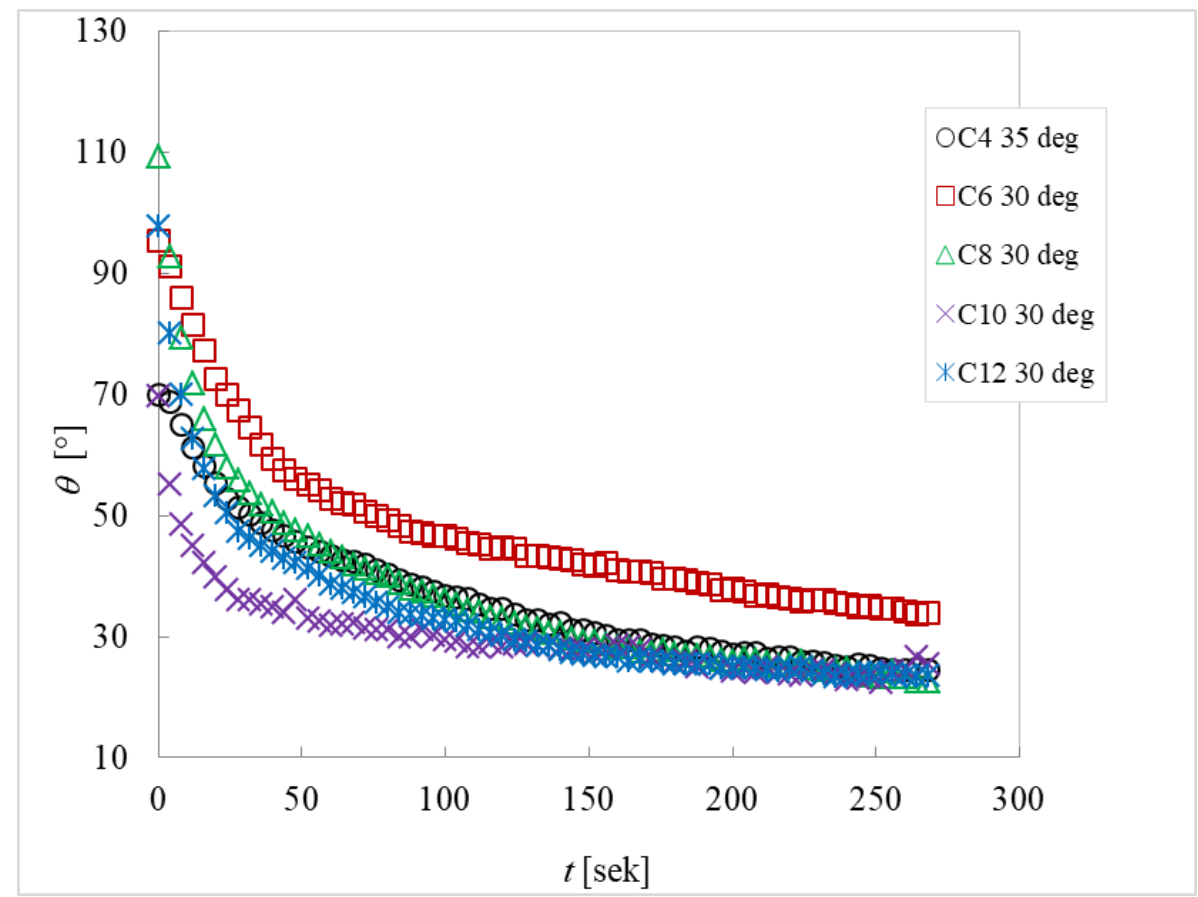

Figure S13. Contact angle of "sweet" ionic liquids on Scots pine wood. 


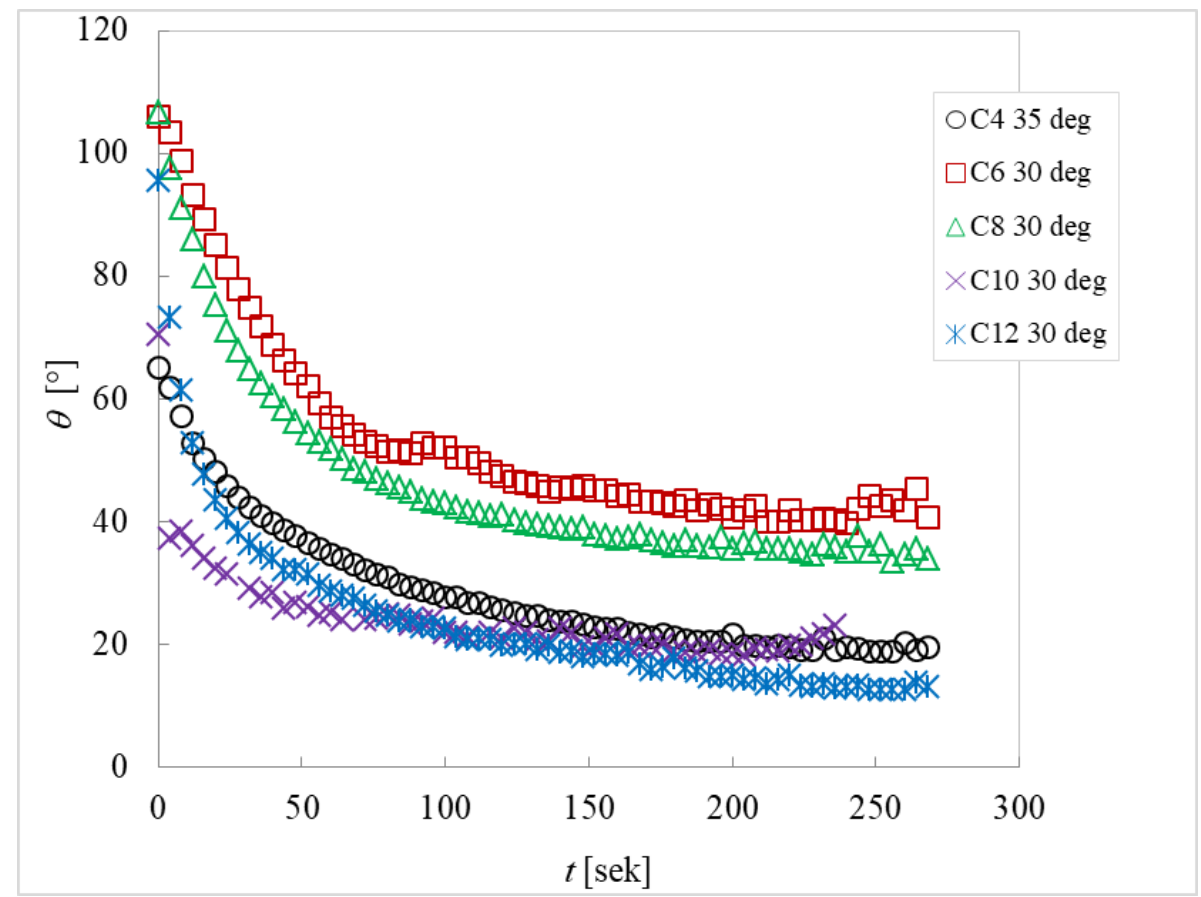

Figure S14. Contact angle of "sweet" ionic liquids on beech wood. 
NMR spectra of the discussed CILs in $\mathrm{CDCl}_{3}$
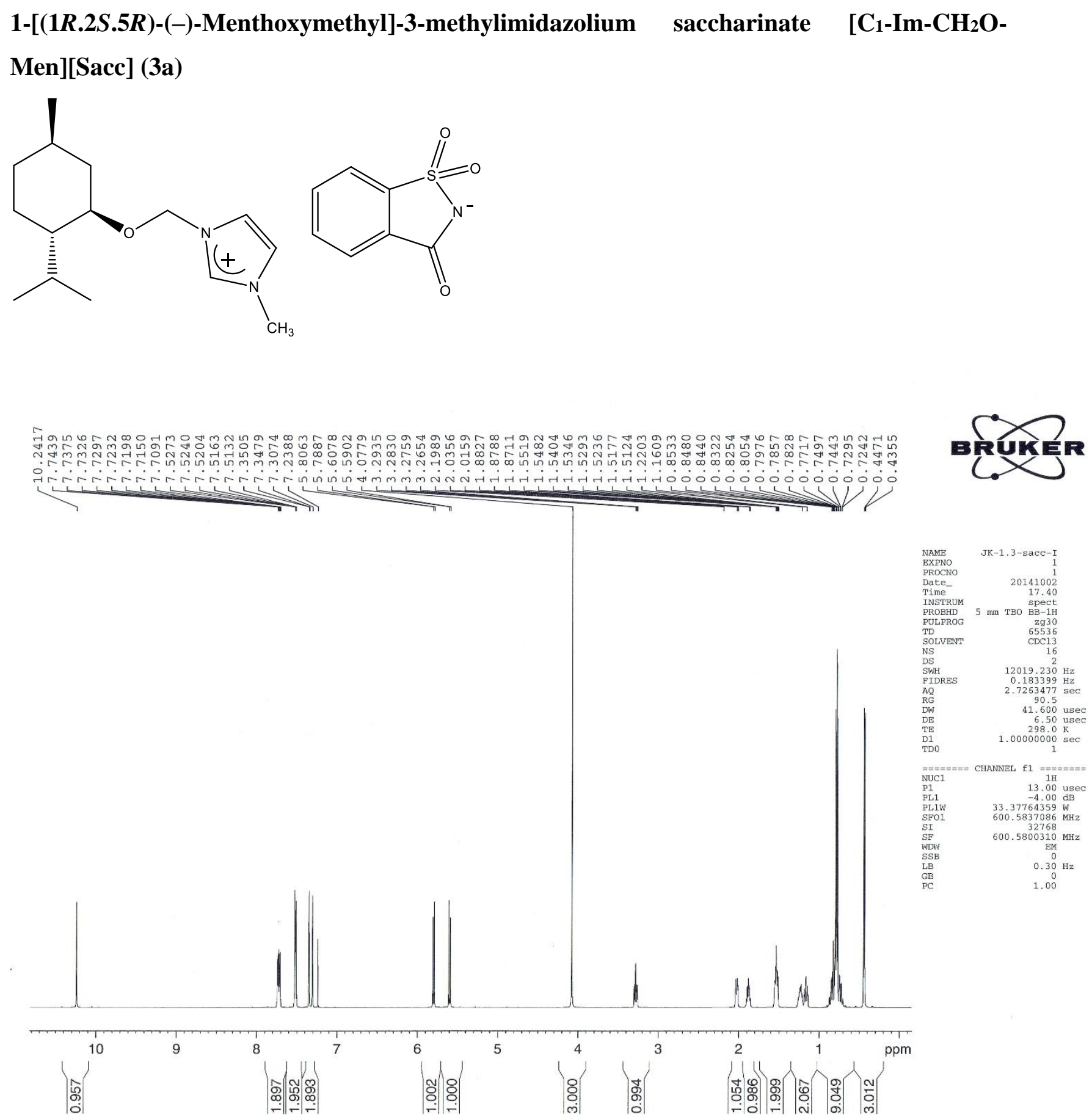

Figure S15. 


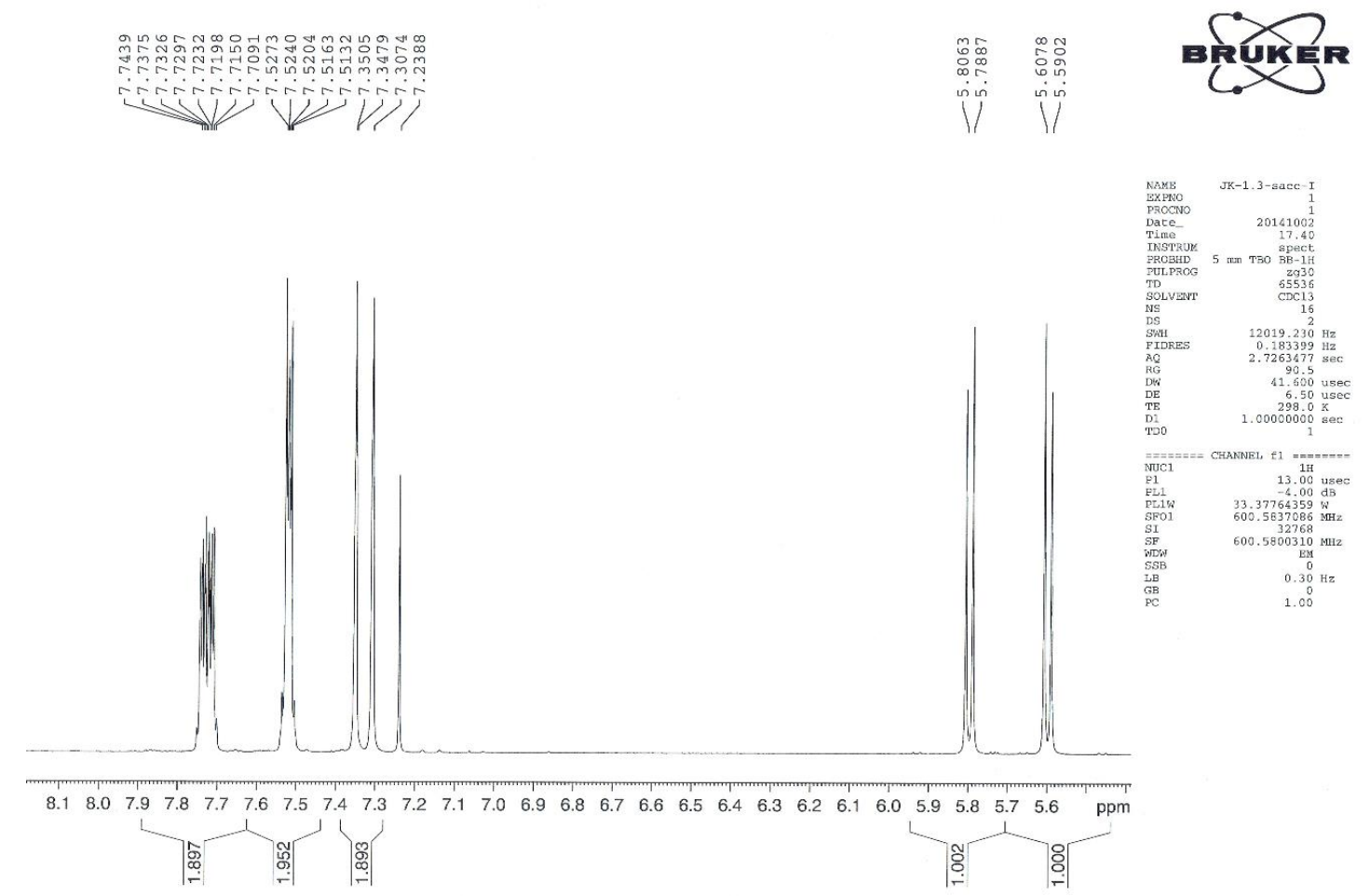

Figure S16.

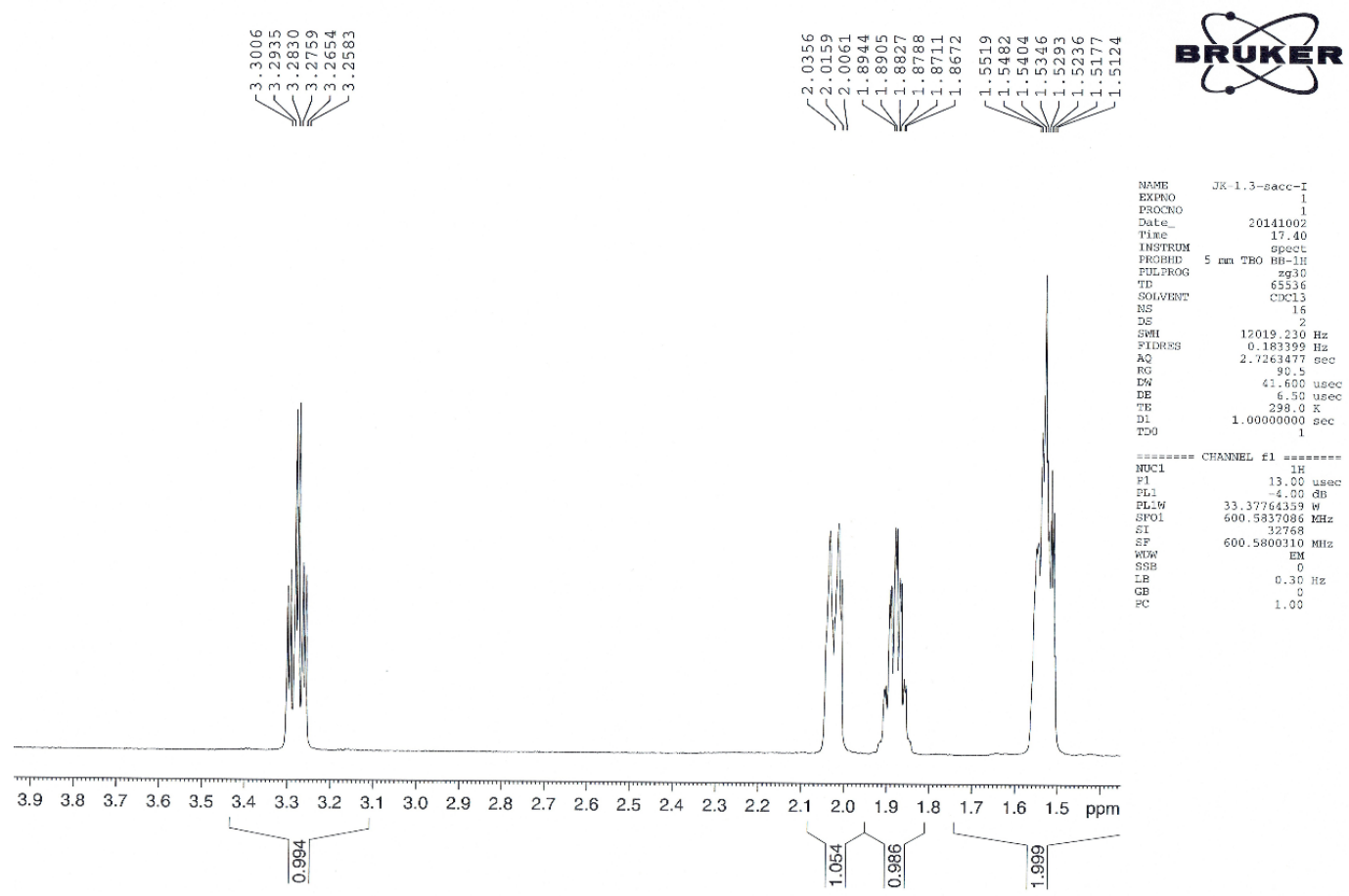

Figure S17. 


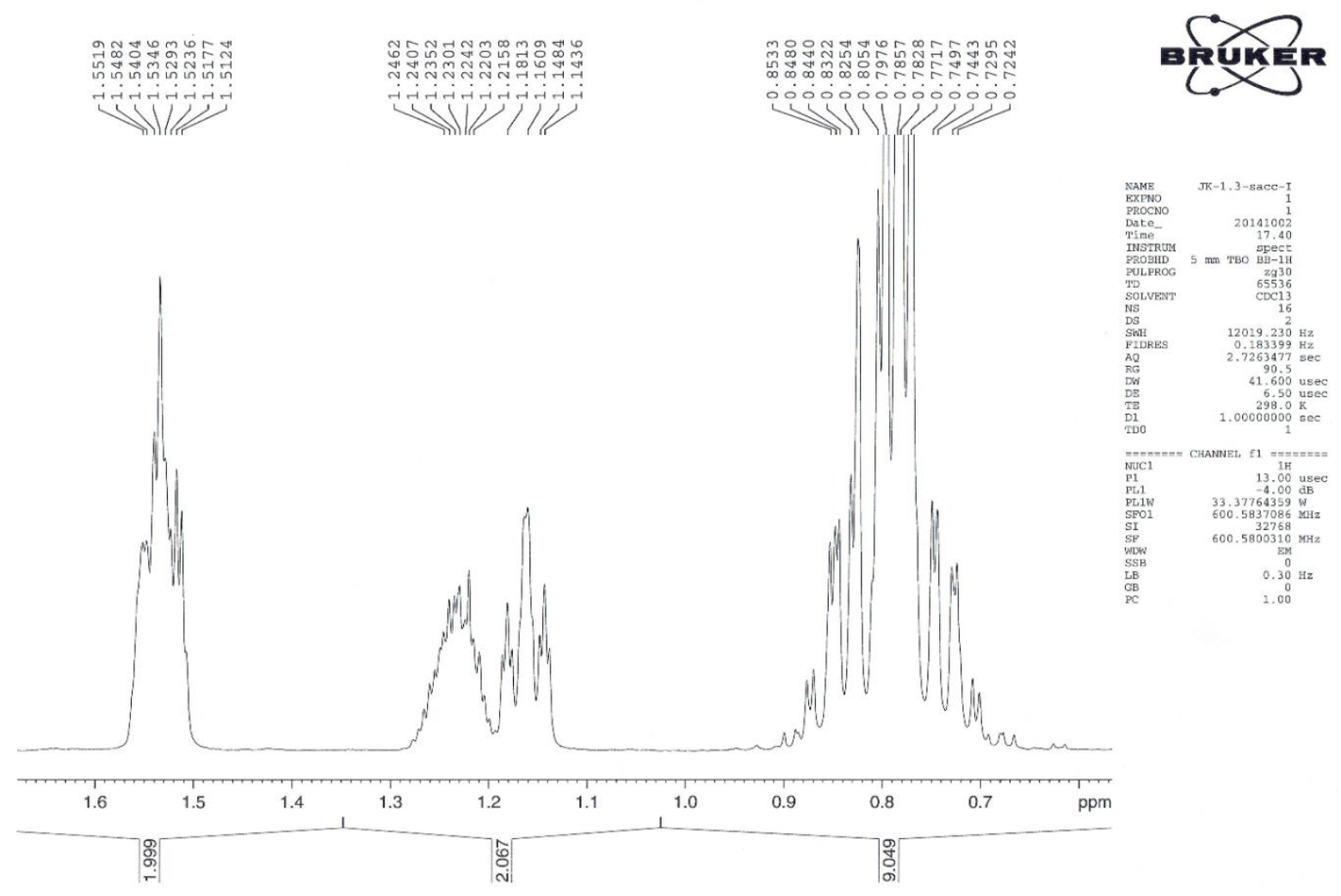

Figure S18.

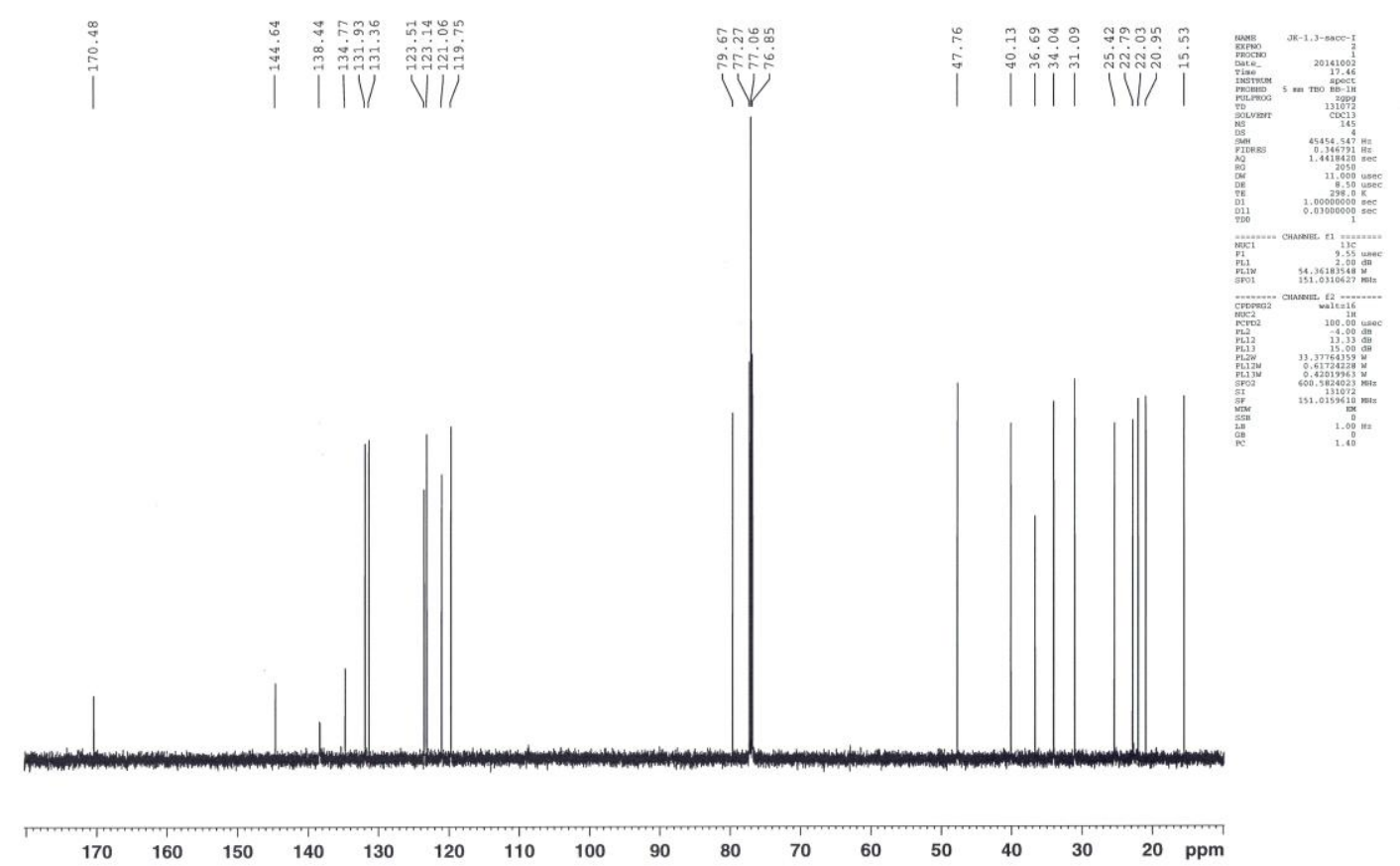

Figure S19. 
3-Ethyl-1-[(1R.2S.5R)-(-)-menthoxymethyl]imidazolium saccharinate $\left[\mathrm{C}_{2}-\mathrm{Im}-\mathrm{CH}_{2} \mathrm{O}\right.$ Men][Sacc] (3b)

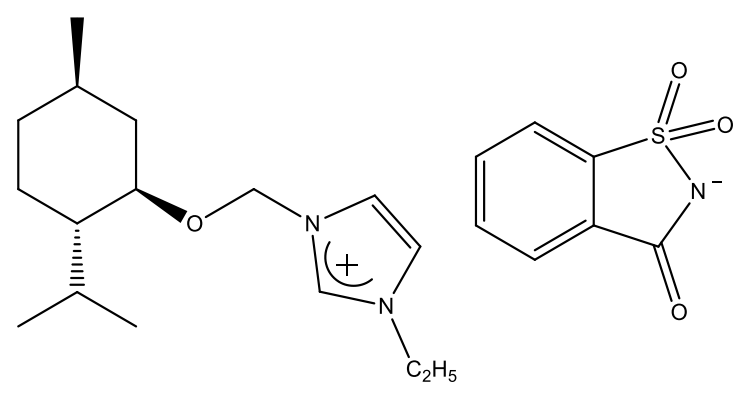

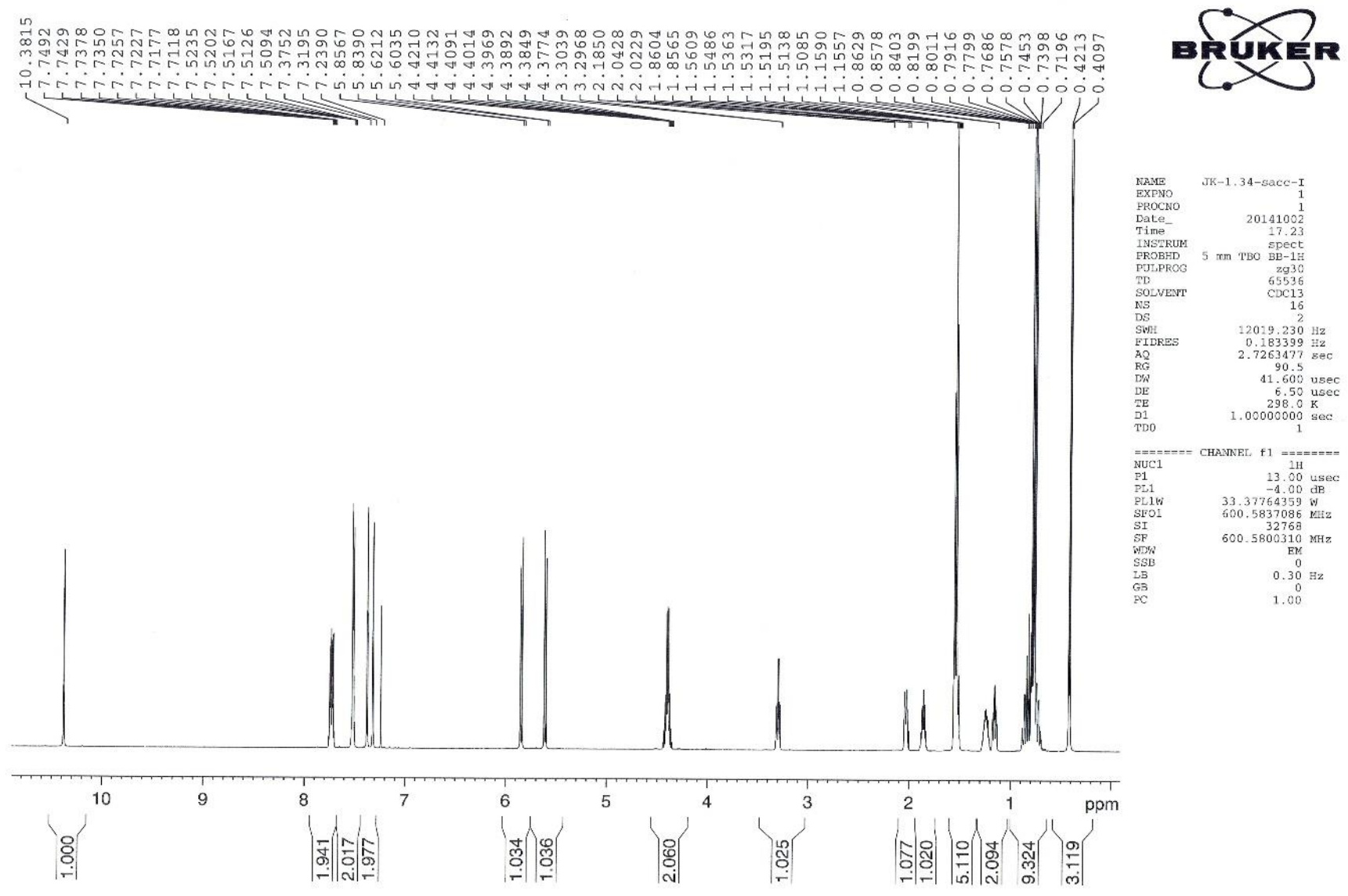

Figure S20. 

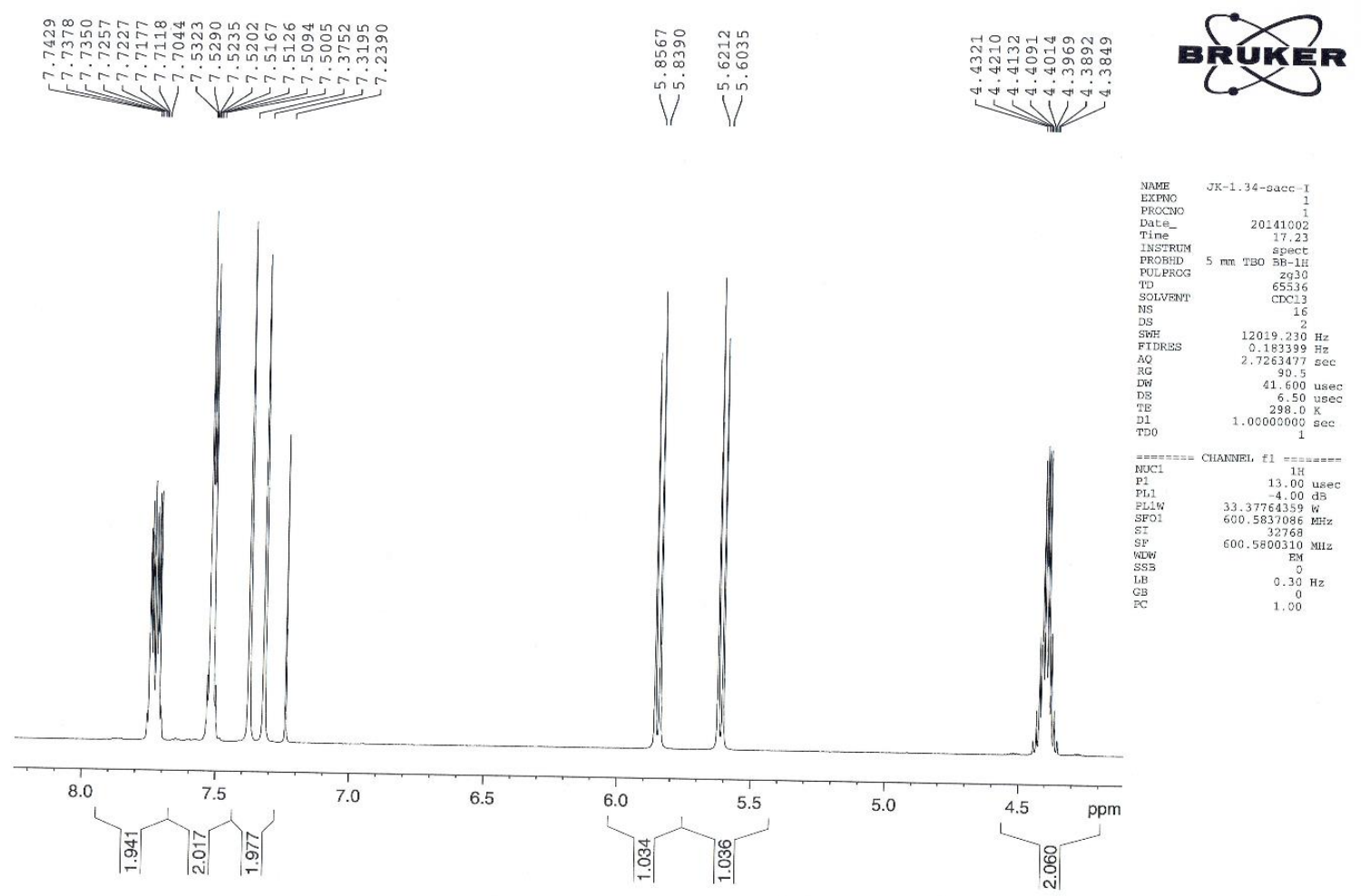

Figure S21.
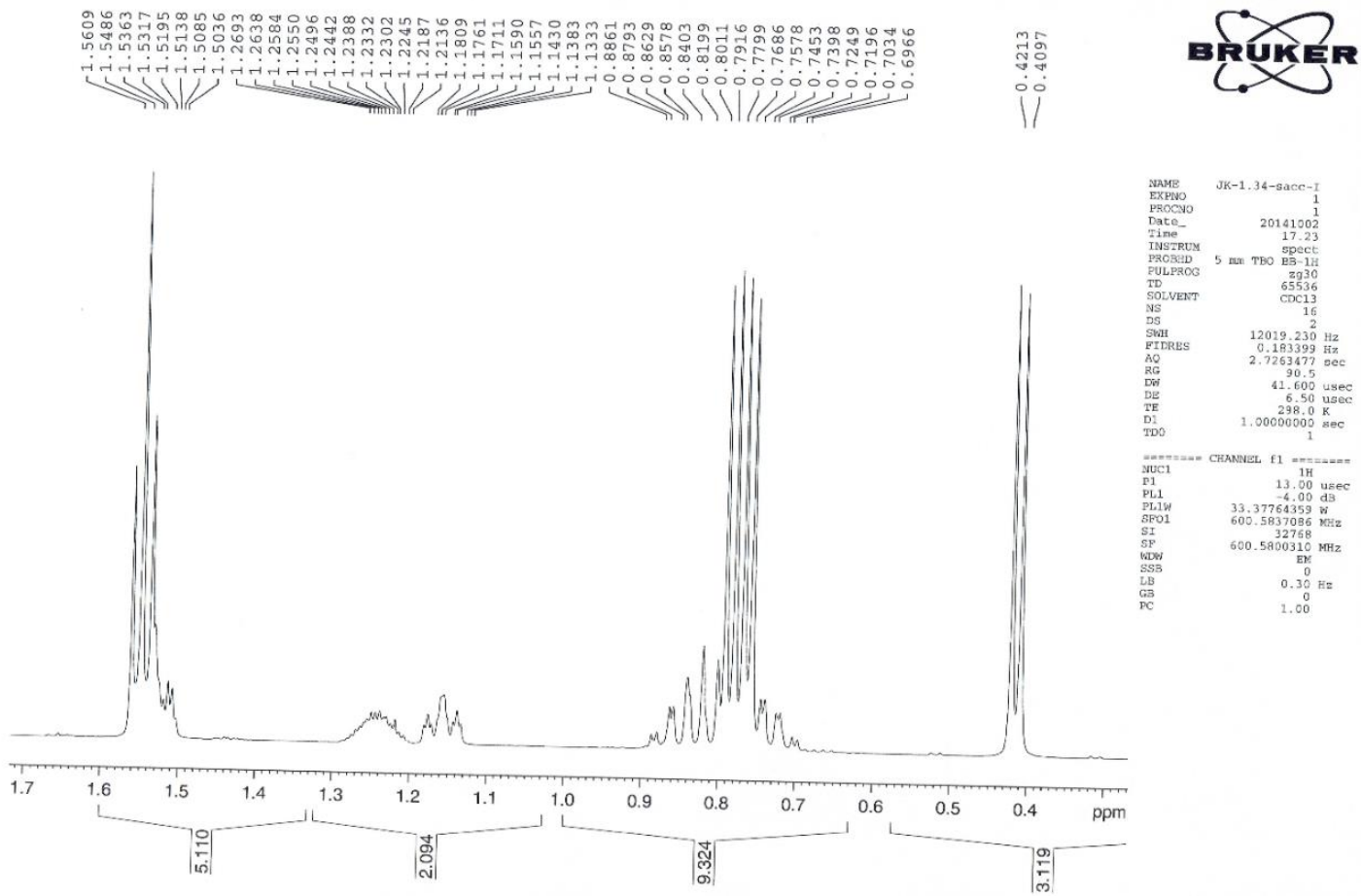

Figure S22. 


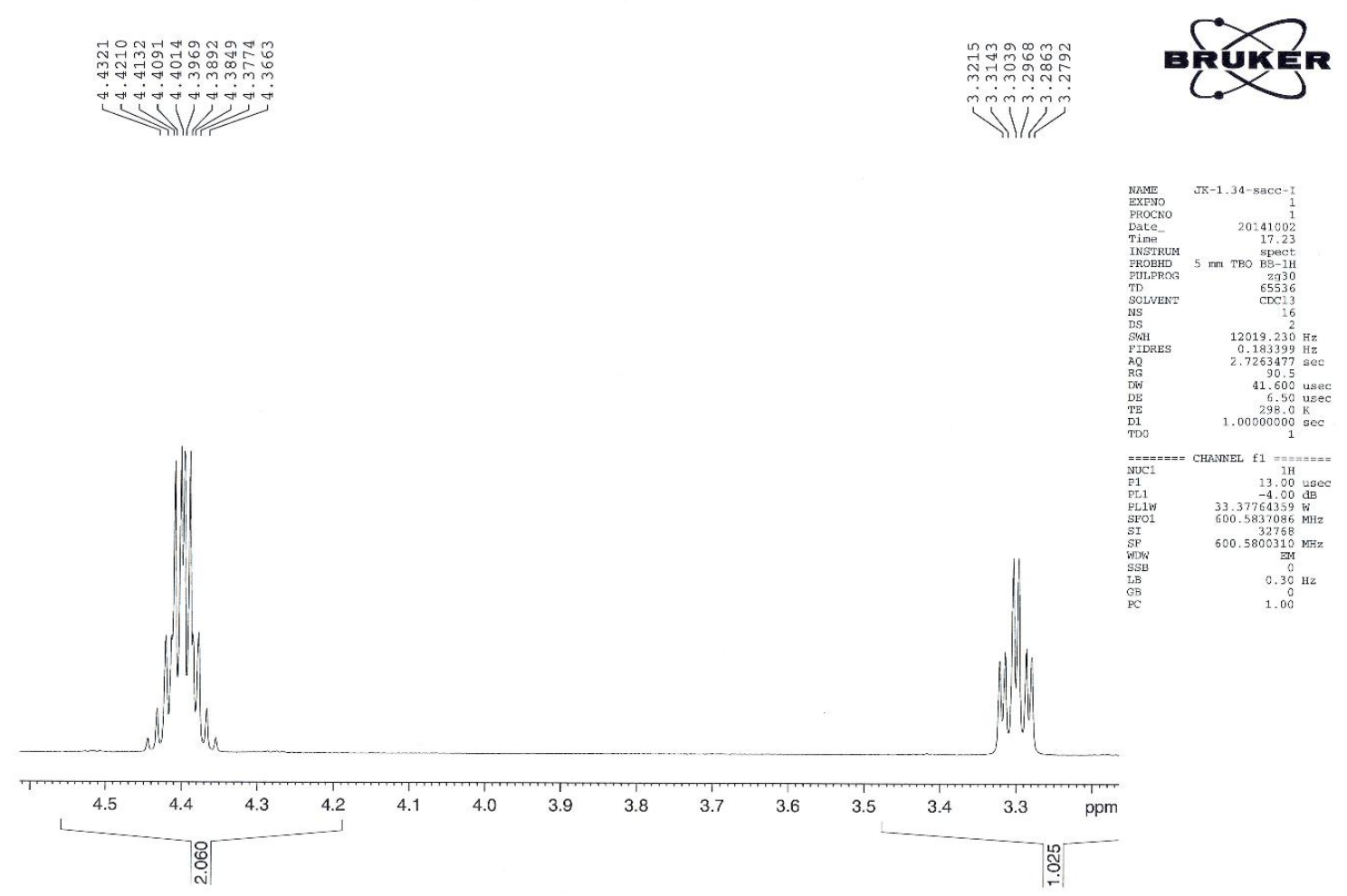

Figure S23.
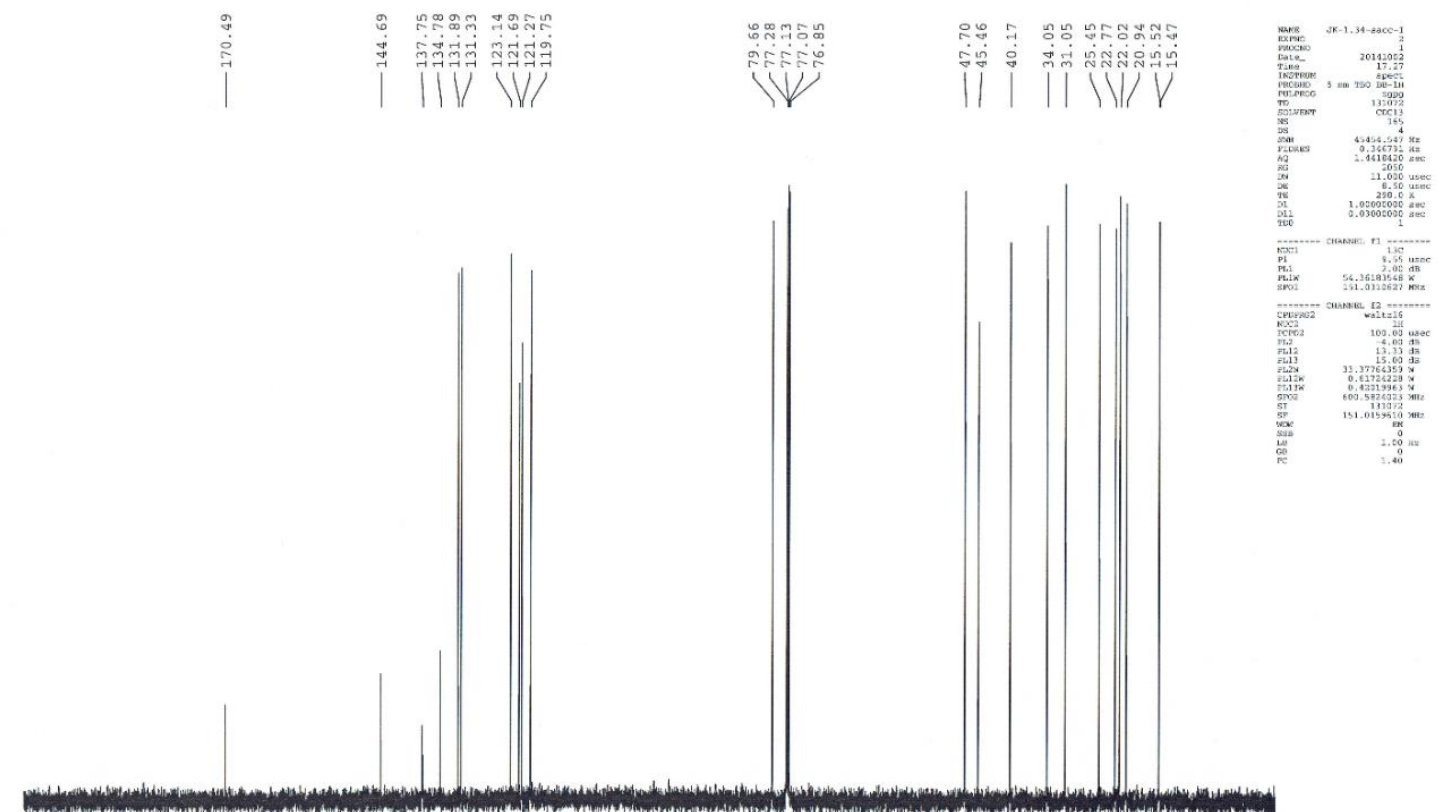

$\begin{array}{lllllllllllllllllllll}200 & 190 & 180 & 170 & 160 & 150 & 140 & 130 & 120 & 110 & 100 & 90 & 80 & 70 & 60 & 50 & 40 & 30 & 20 & 10 & \mathrm{ppm}\end{array}$

Figure S24. 
1-[(1R.2S.5R)-(-)-Menthoxymethyl]-3-propylimidazolium saccharinate $\left[\mathrm{C}_{3}-\mathrm{Im}-\mathrm{CH}_{2} \mathrm{O}\right.$ Men][Sacc] (3c)

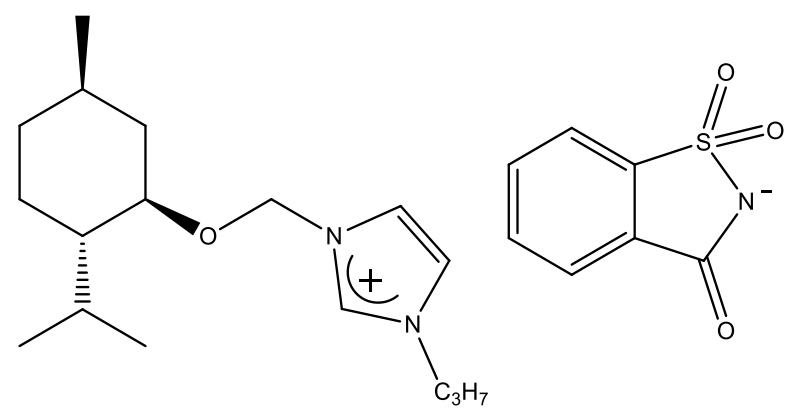

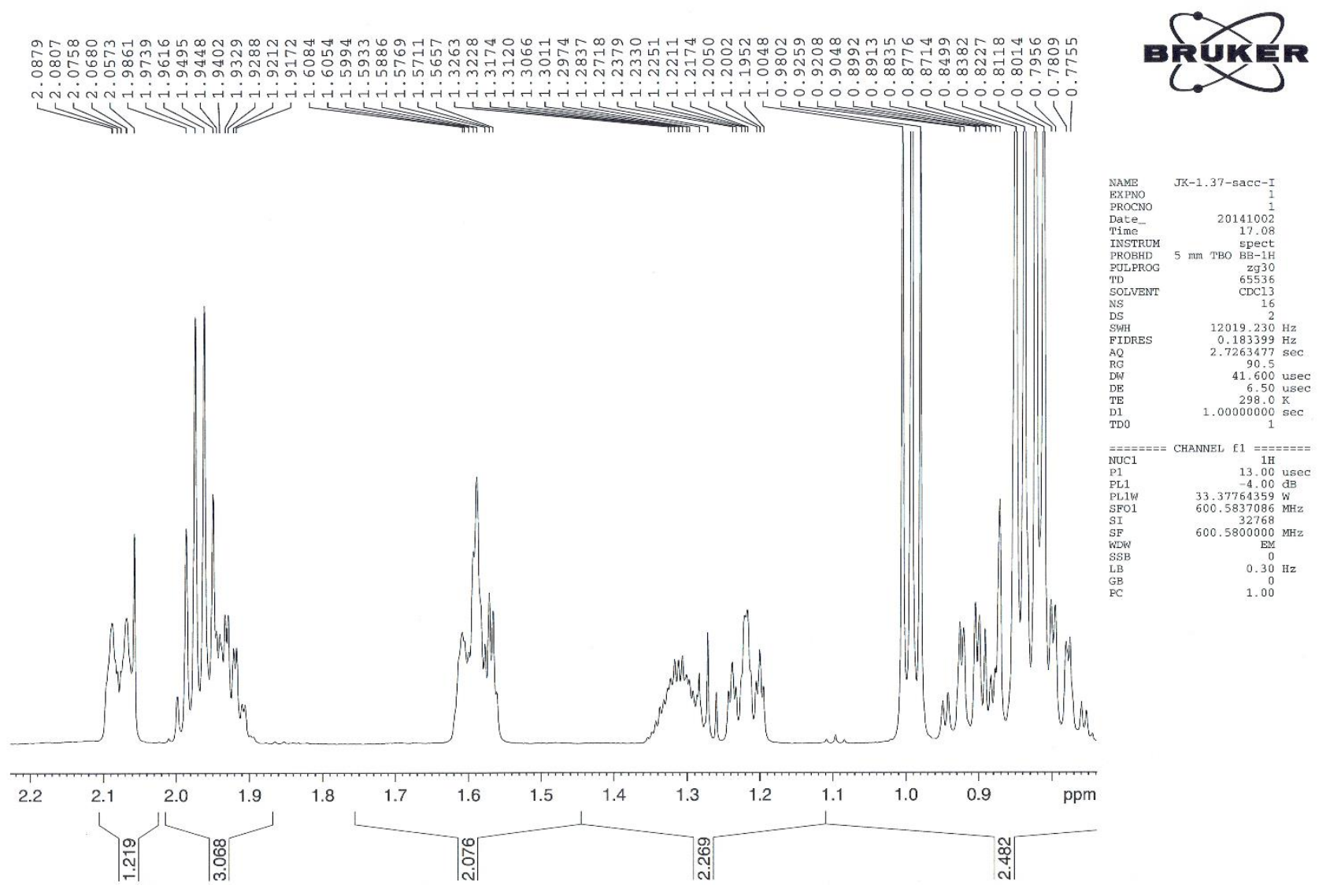

Figure S25. 

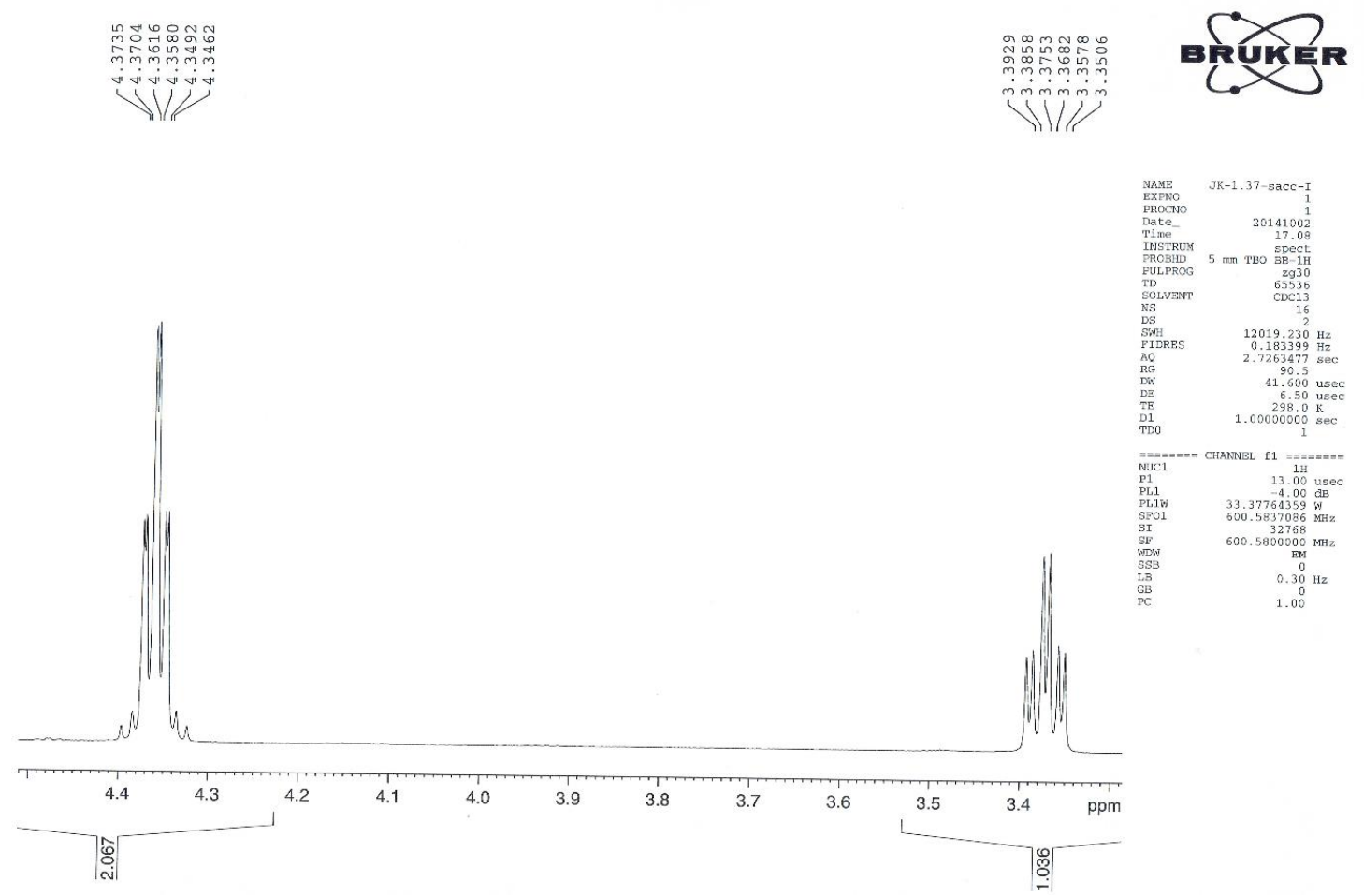

Figure S26.

Figure

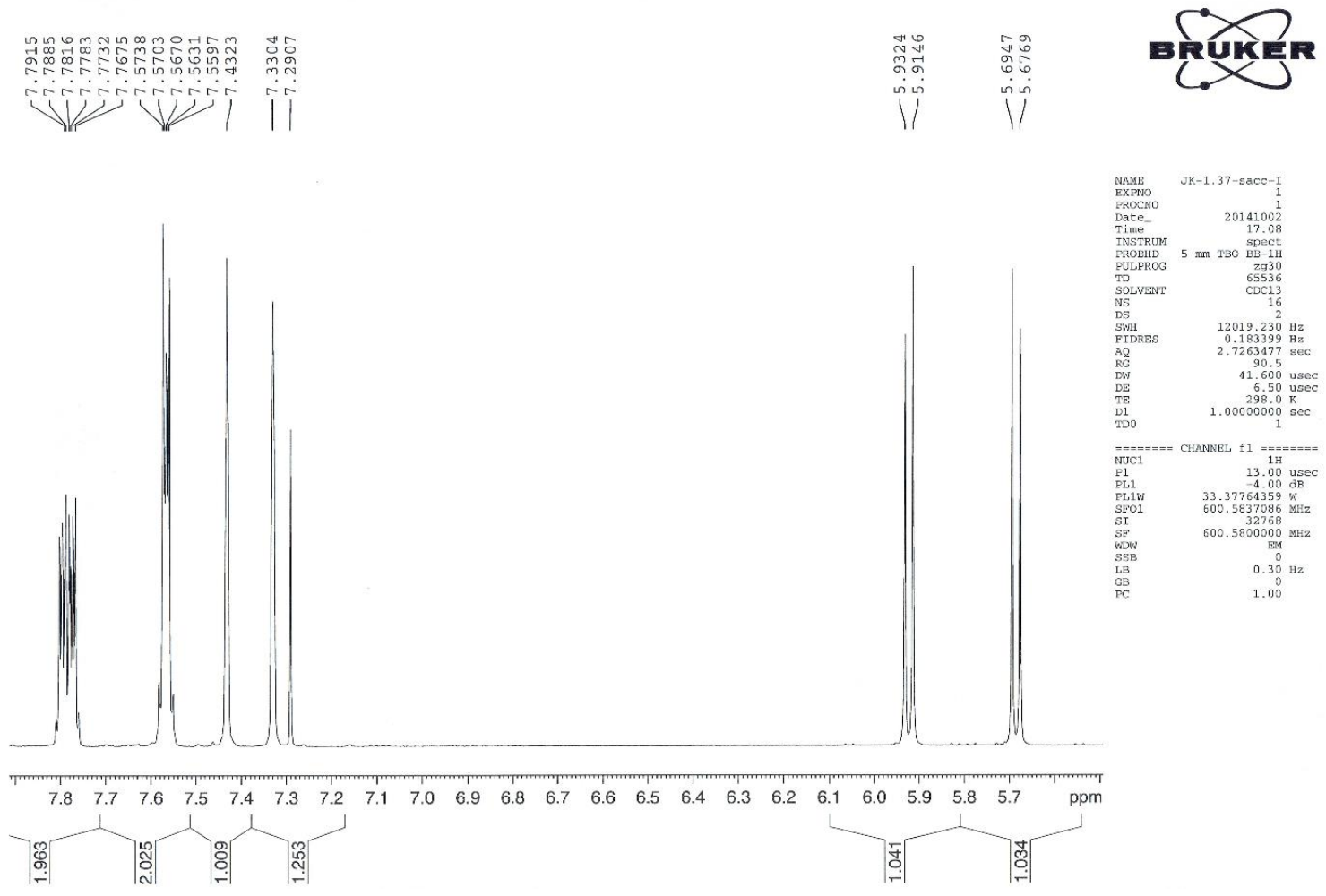

Figure S27. 


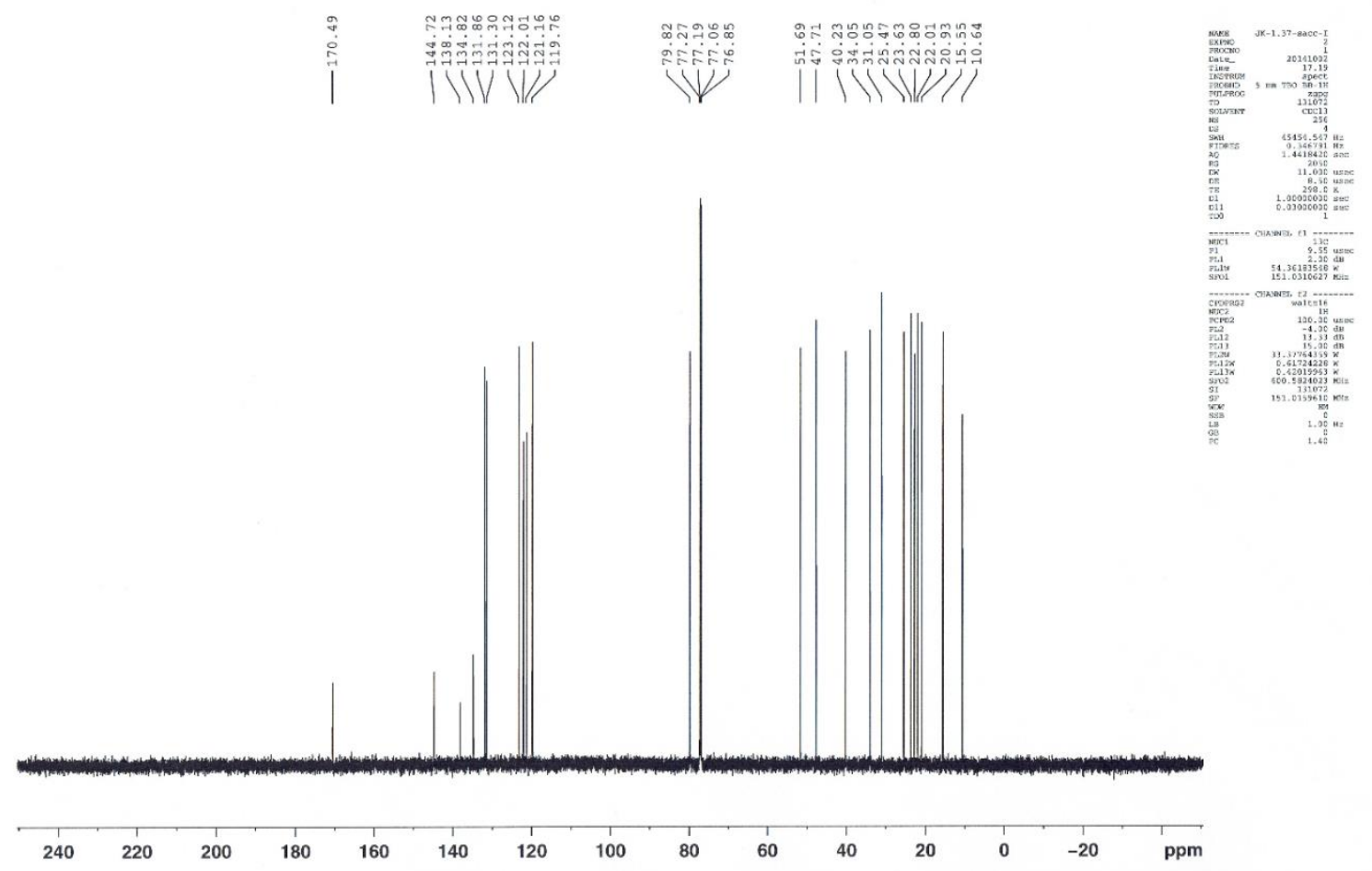

Figure S28.

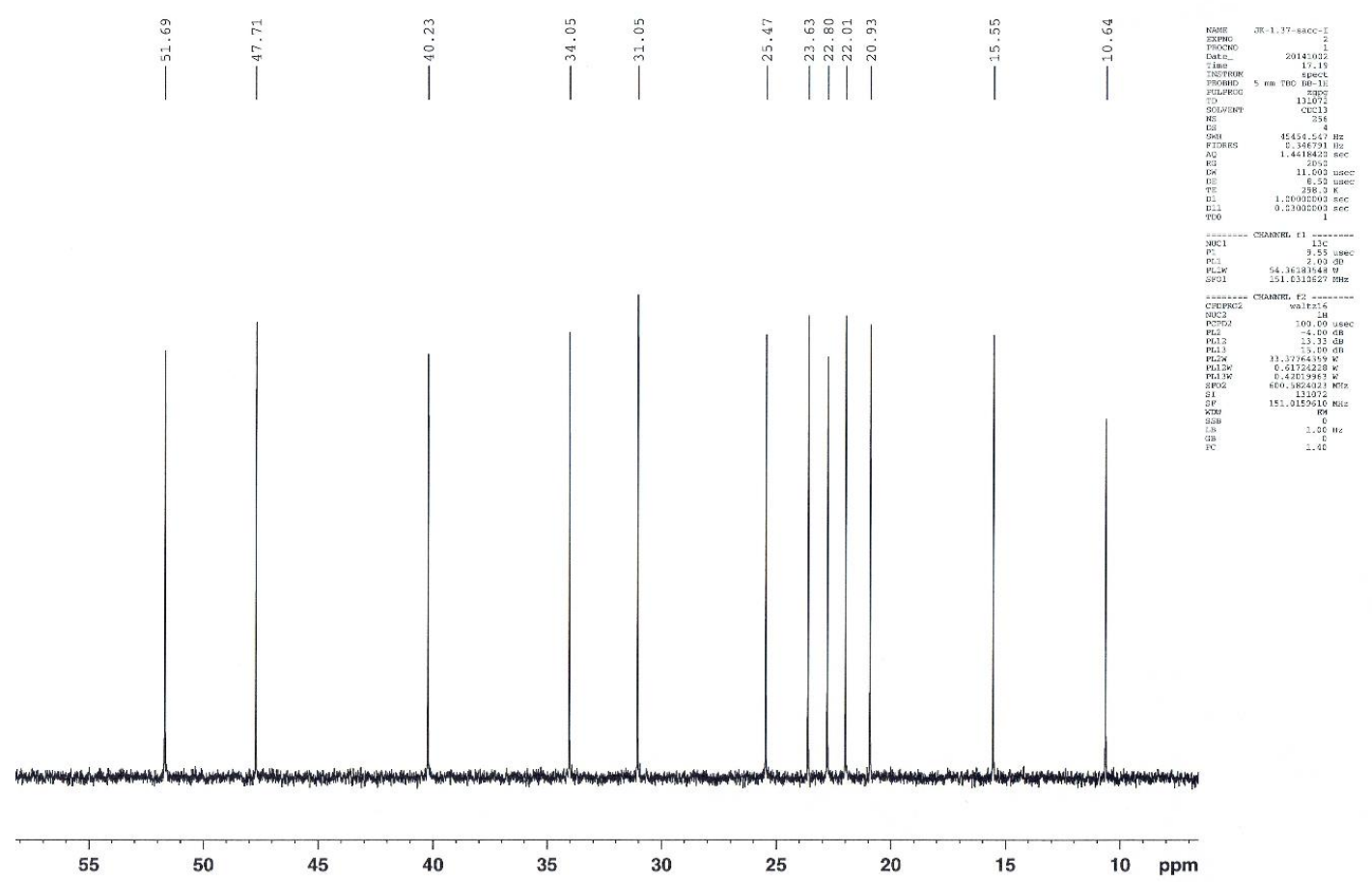

Figure S29. 
3-Butyl-1-[(1R.2S.5R)-(-)-menthoxymethyl]imidazolium saccharinate $\left[\mathrm{C}_{4}-\mathrm{Im}-\mathrm{CH}_{2} \mathrm{O}\right.$ Men][Sacc] (3d)<smiles>[CH][n+]1ccn(CO[C@H]2C[C@H](C)CC[C@H]2C(C)C)c1</smiles>

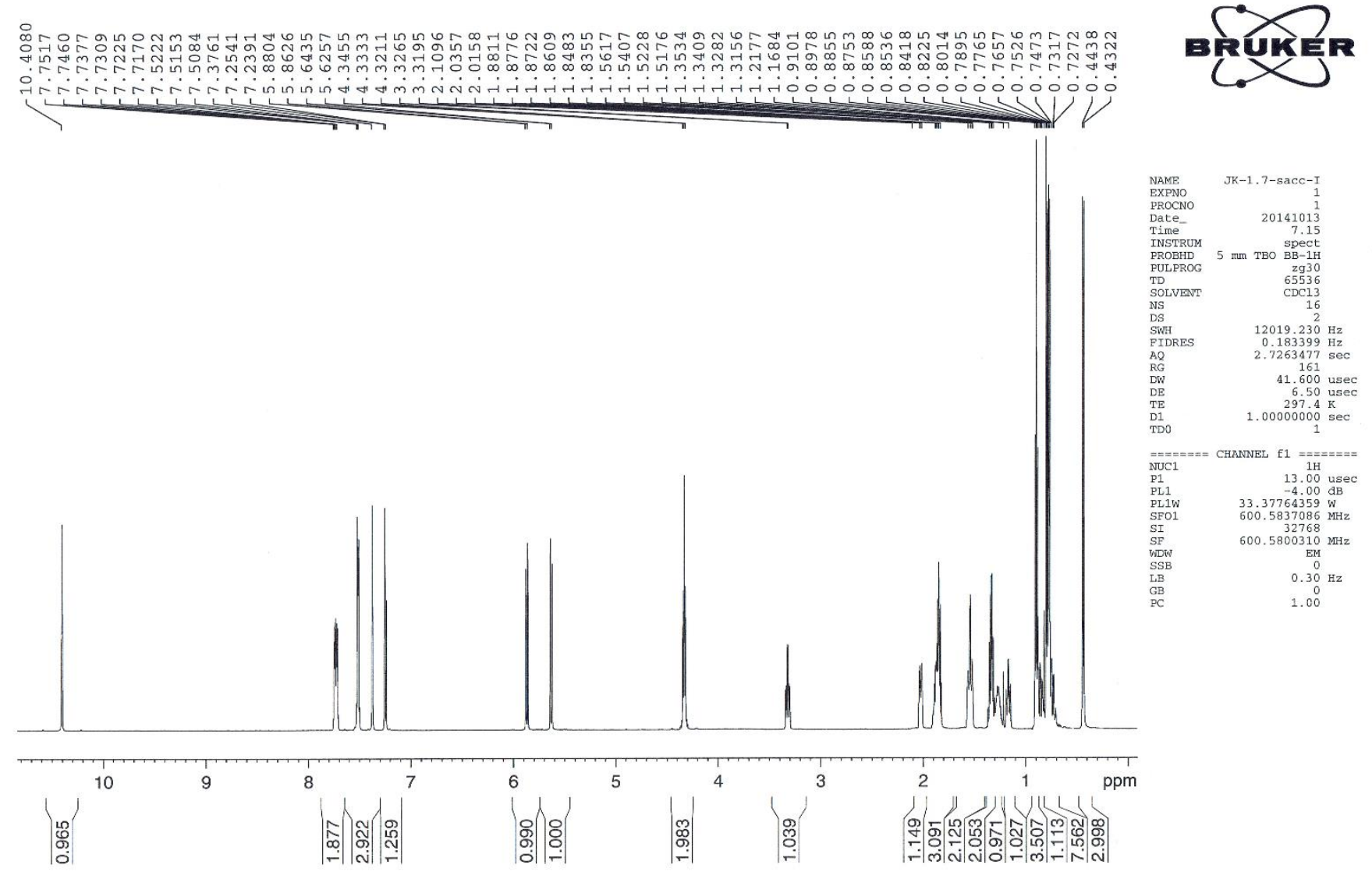

Figure S30. 


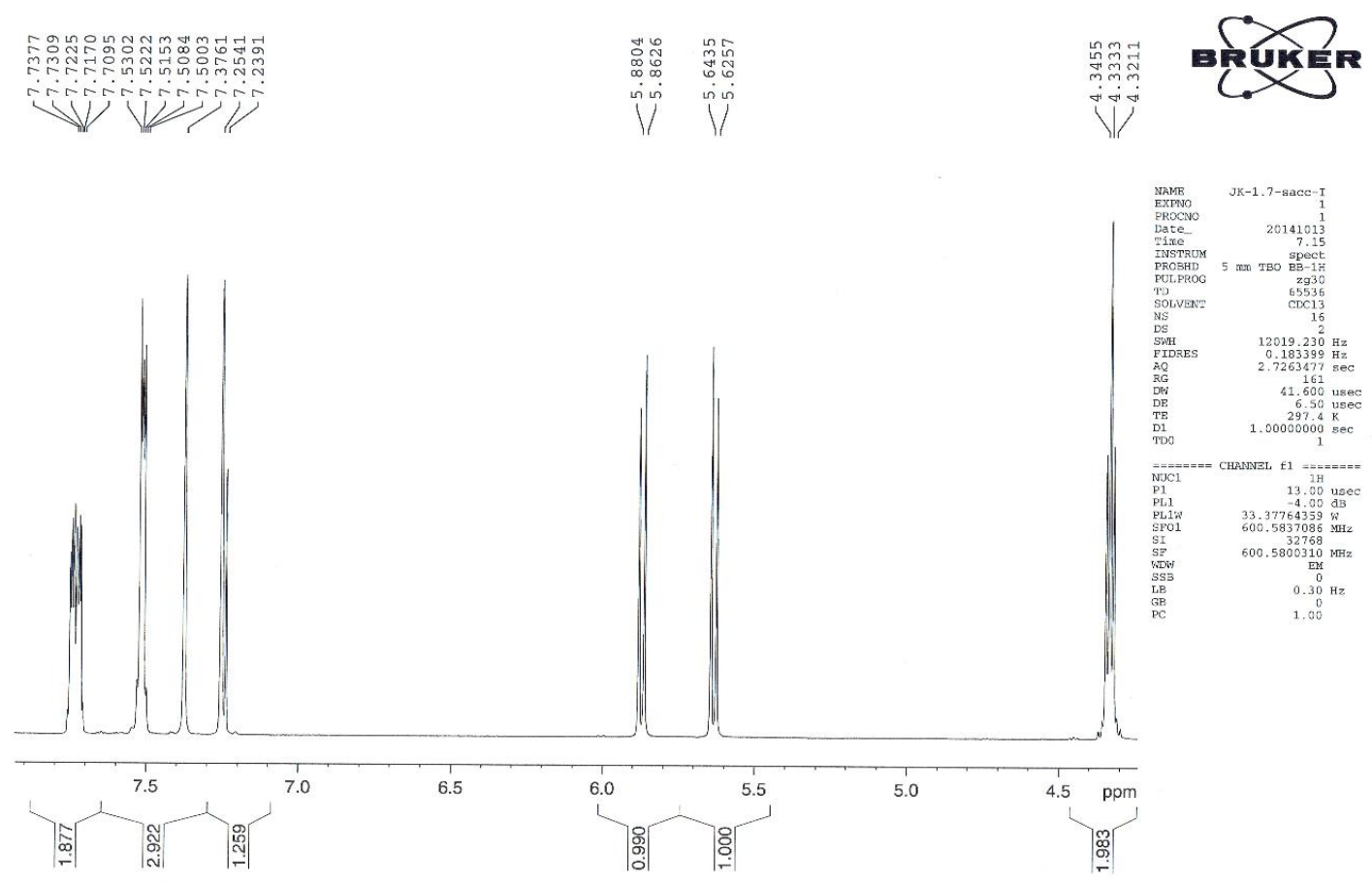

Figure S31.

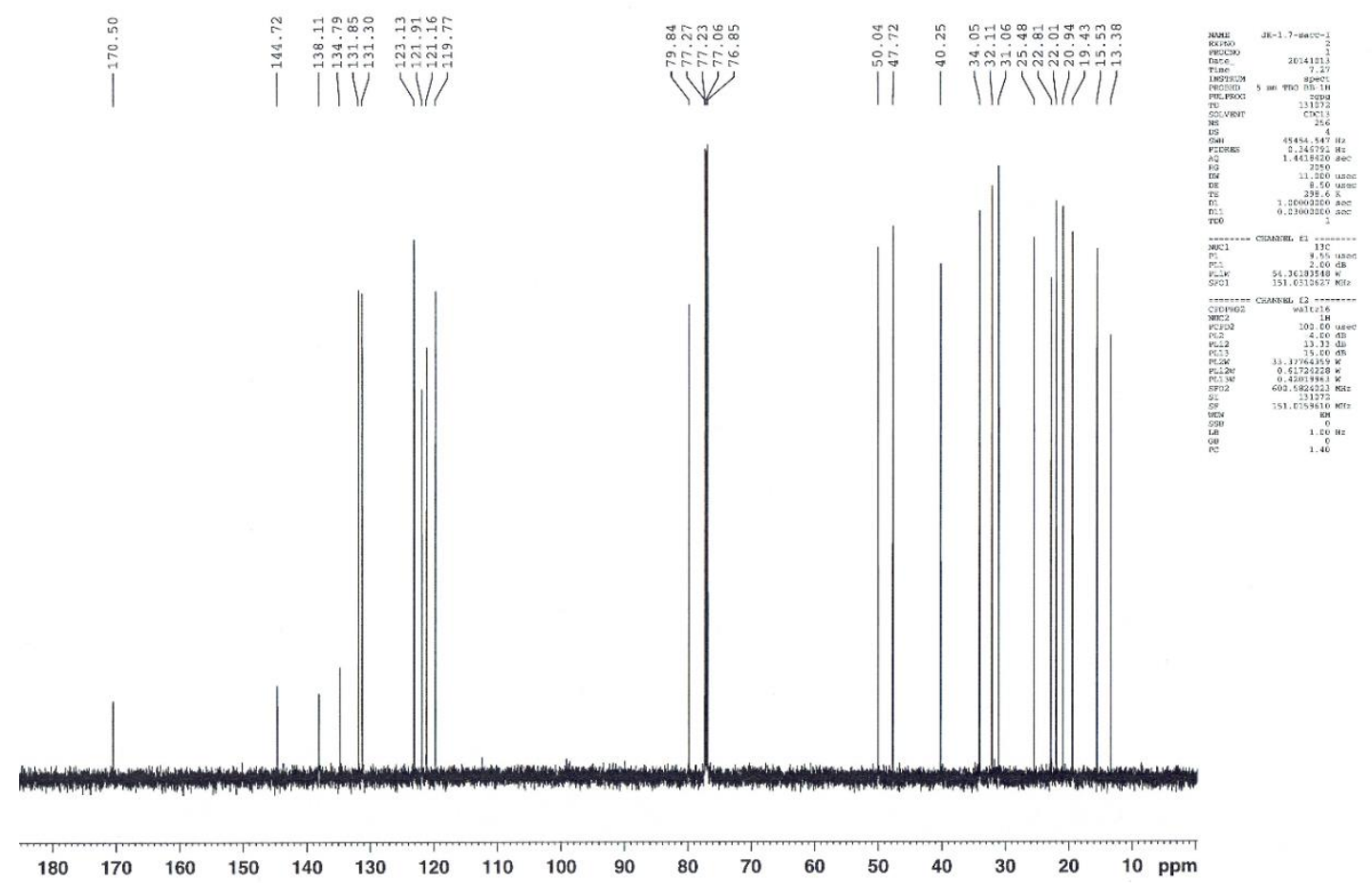

Figure S32. 
$1-[(1 R .2 S .5 R)-(-)-M e n t h o x y m e t h y l]-3-p e n t y l i m i d a z o l i u m ~ s a c c h a r i n a t e ~\left[C_{5}-I m-\mathrm{CH}_{2} \mathrm{O}-\right.$ Men][Sacc] (3e)<smiles></smiles>

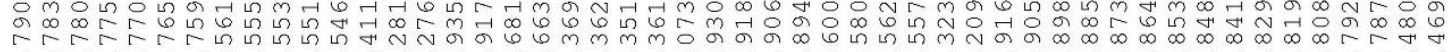

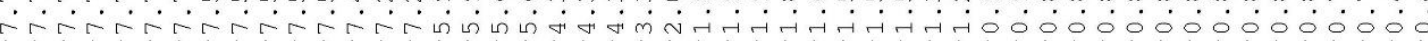

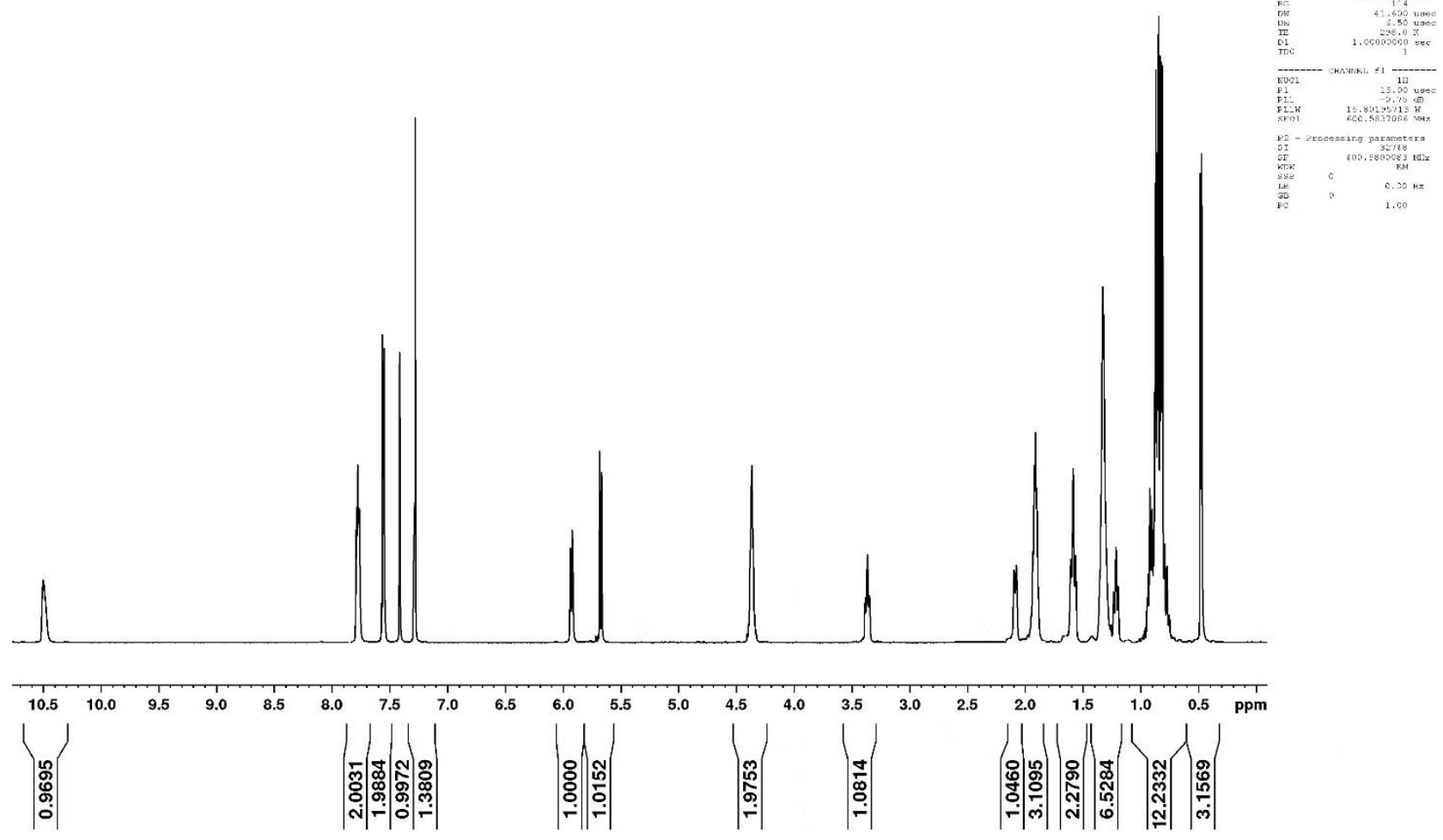

Figure S33. 


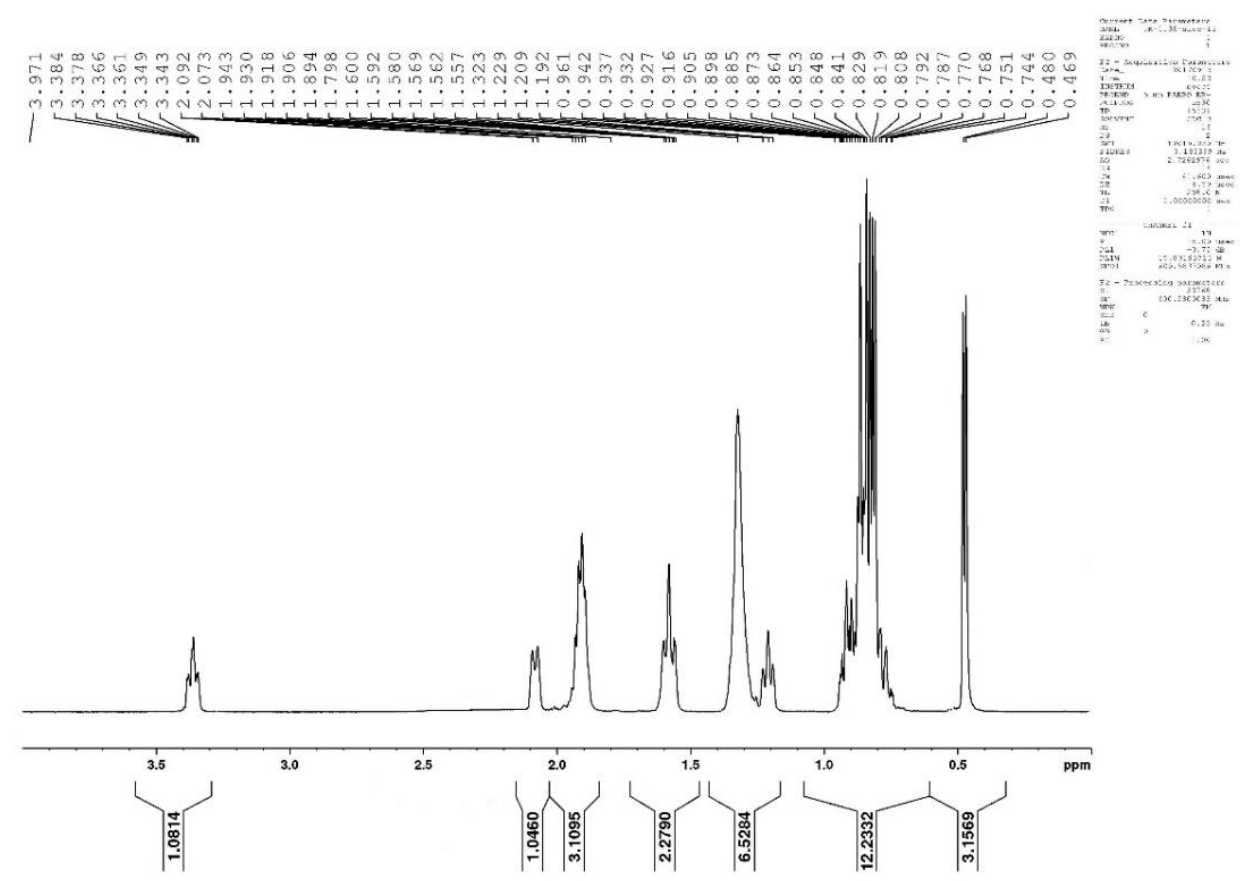

Figure S34.

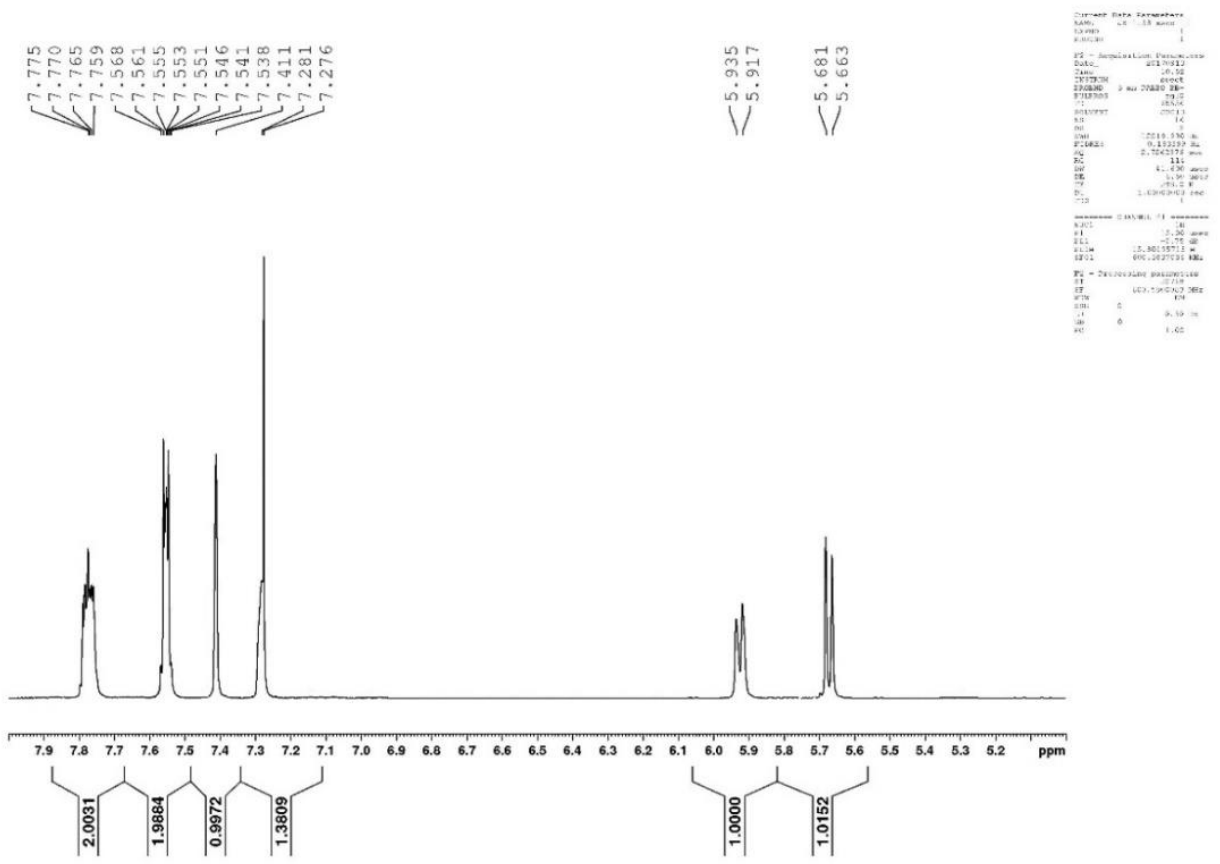

Figure S35. 


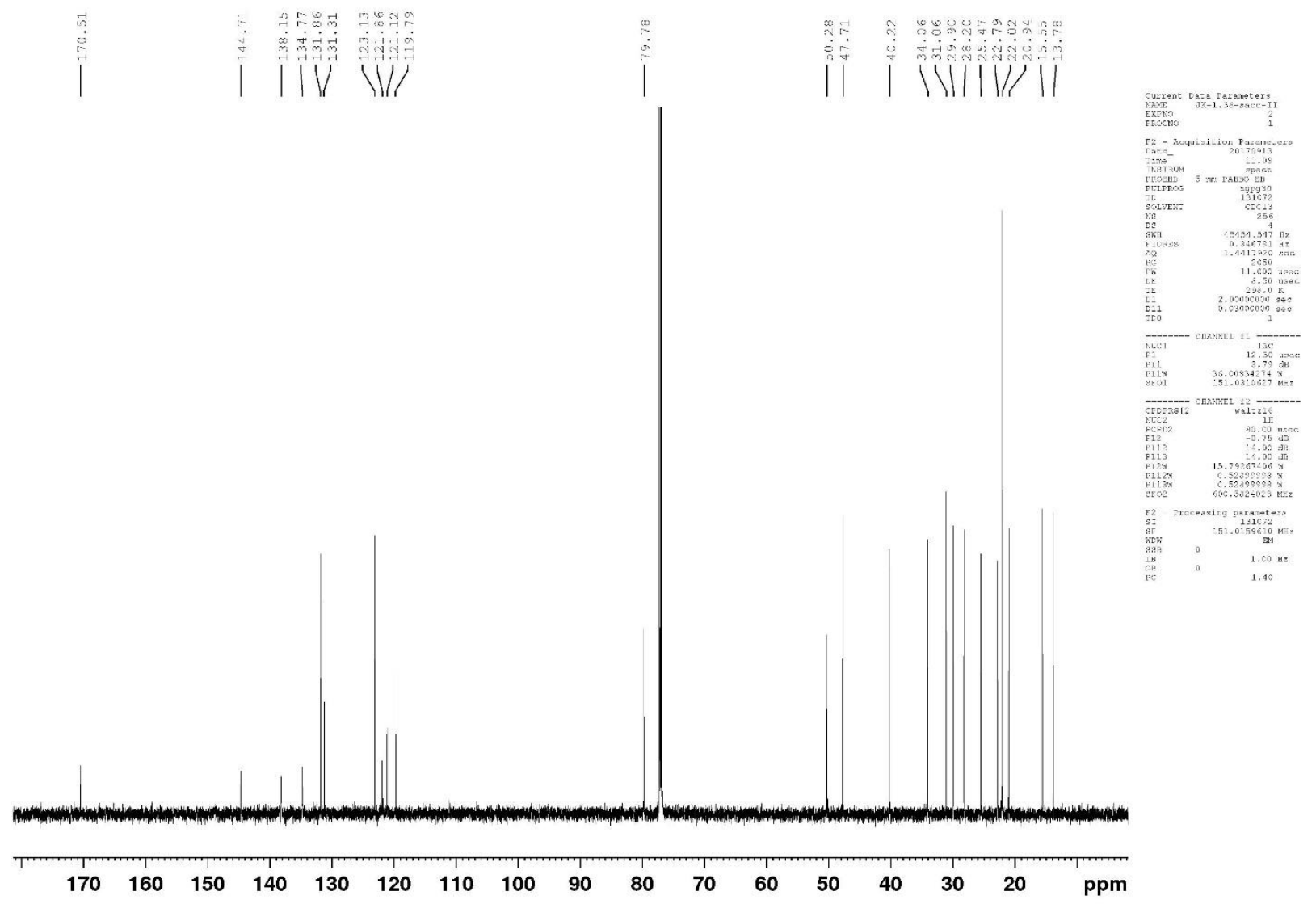

Figure S36. 
3-Hexyl-1-[(1R.2S.5R)-(-)-menthoxymethyl]imidazolium saccharinate [ $\mathrm{C}_{6}-\mathrm{Im}-\mathrm{CH}_{2} \mathrm{O}$ Men][Sacc] (3f)<smiles></smiles>

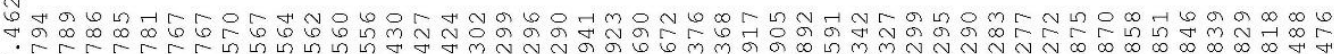

-
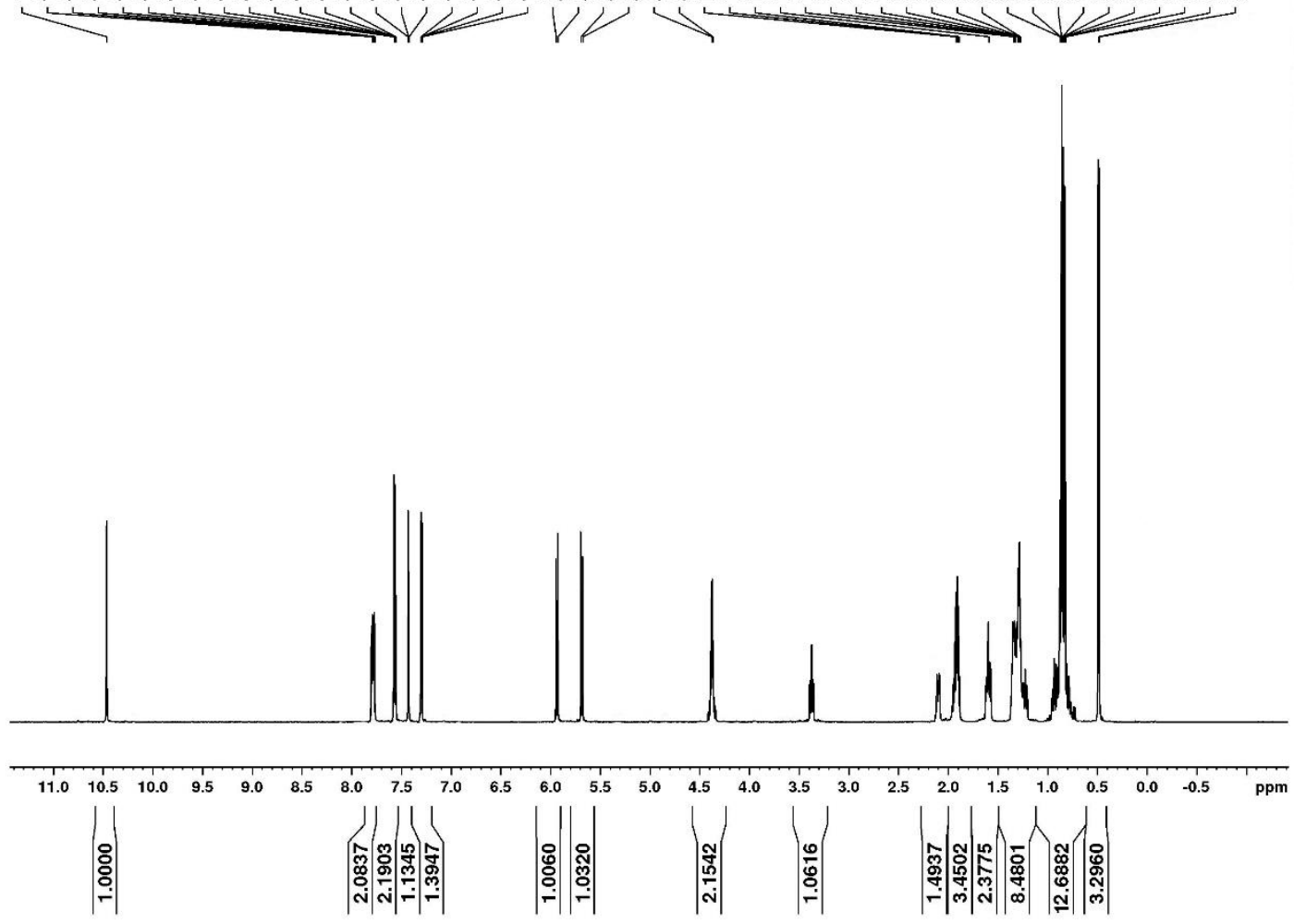

Figure S37. 


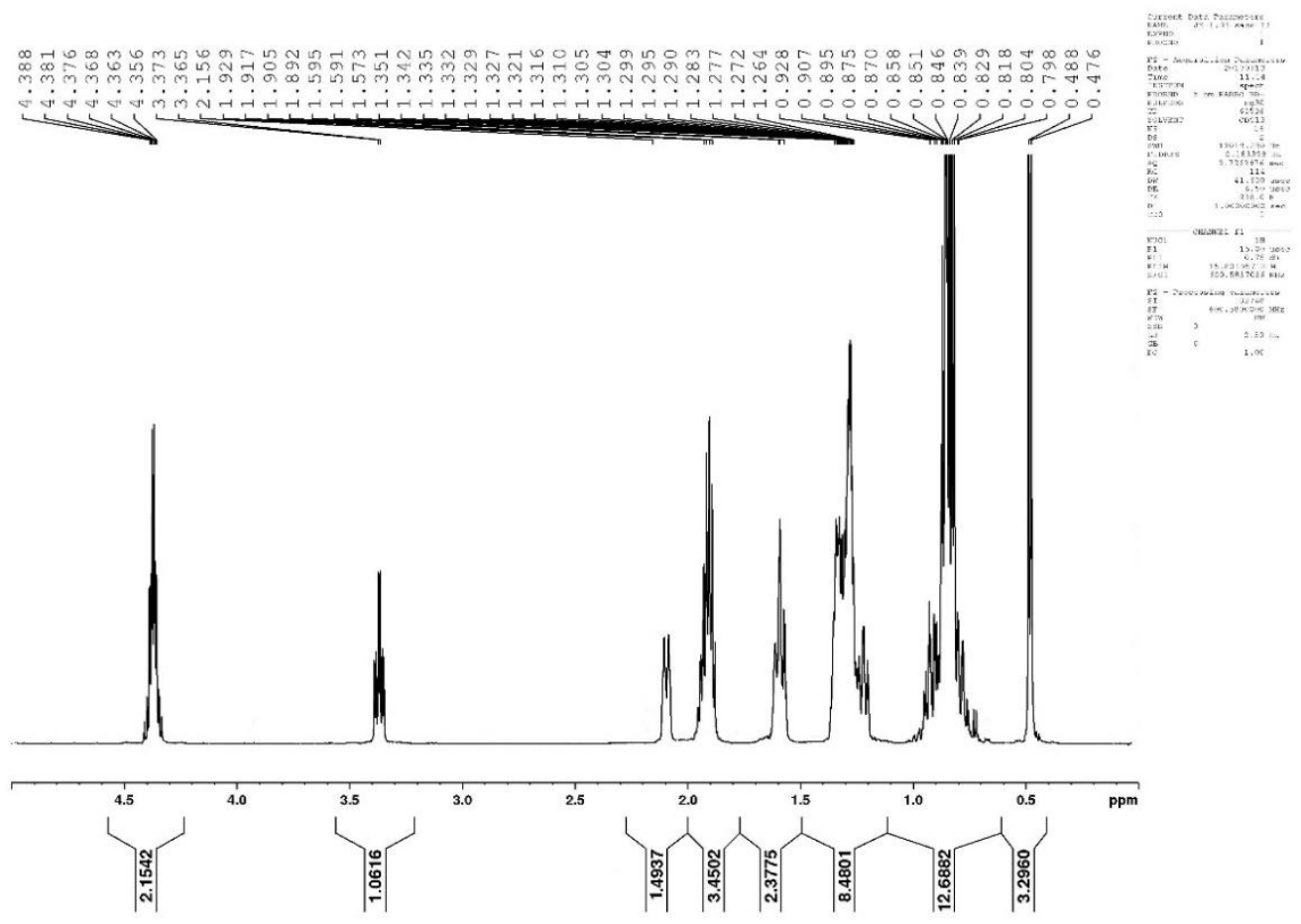

Figure S38.

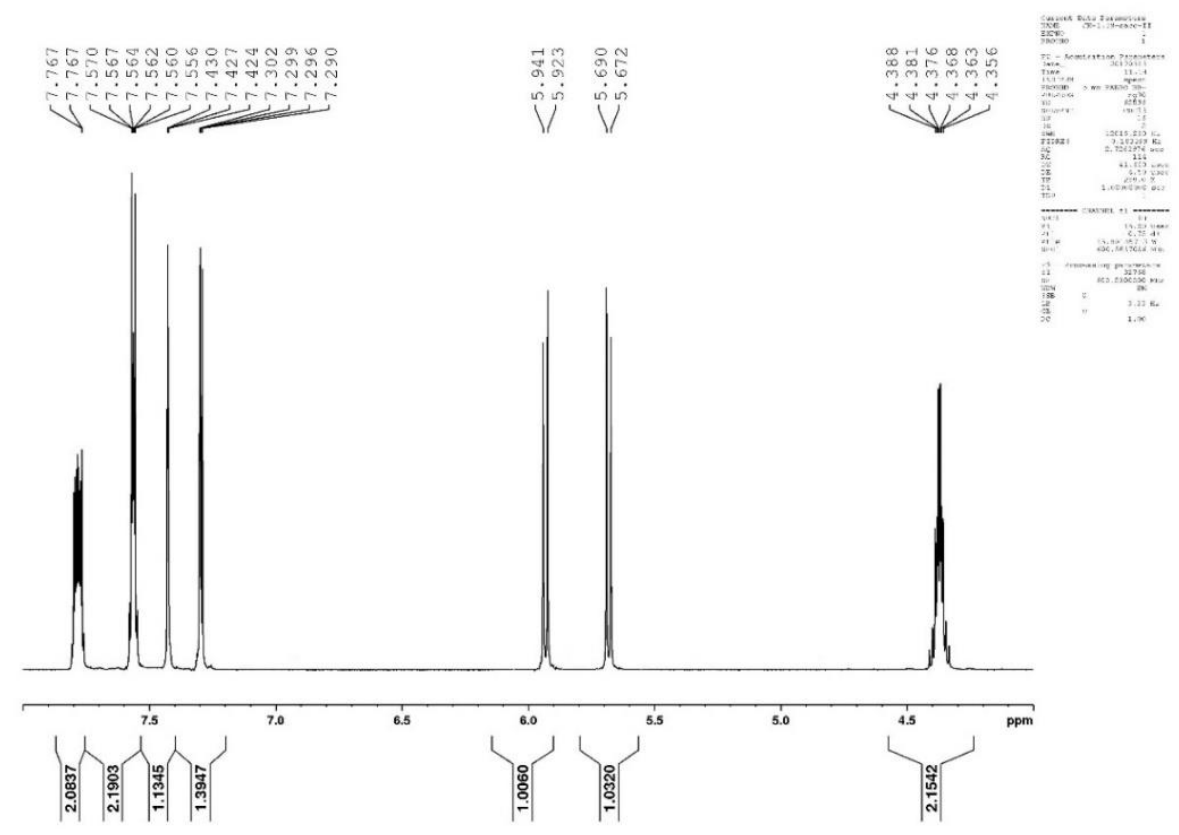

Figure S39. 


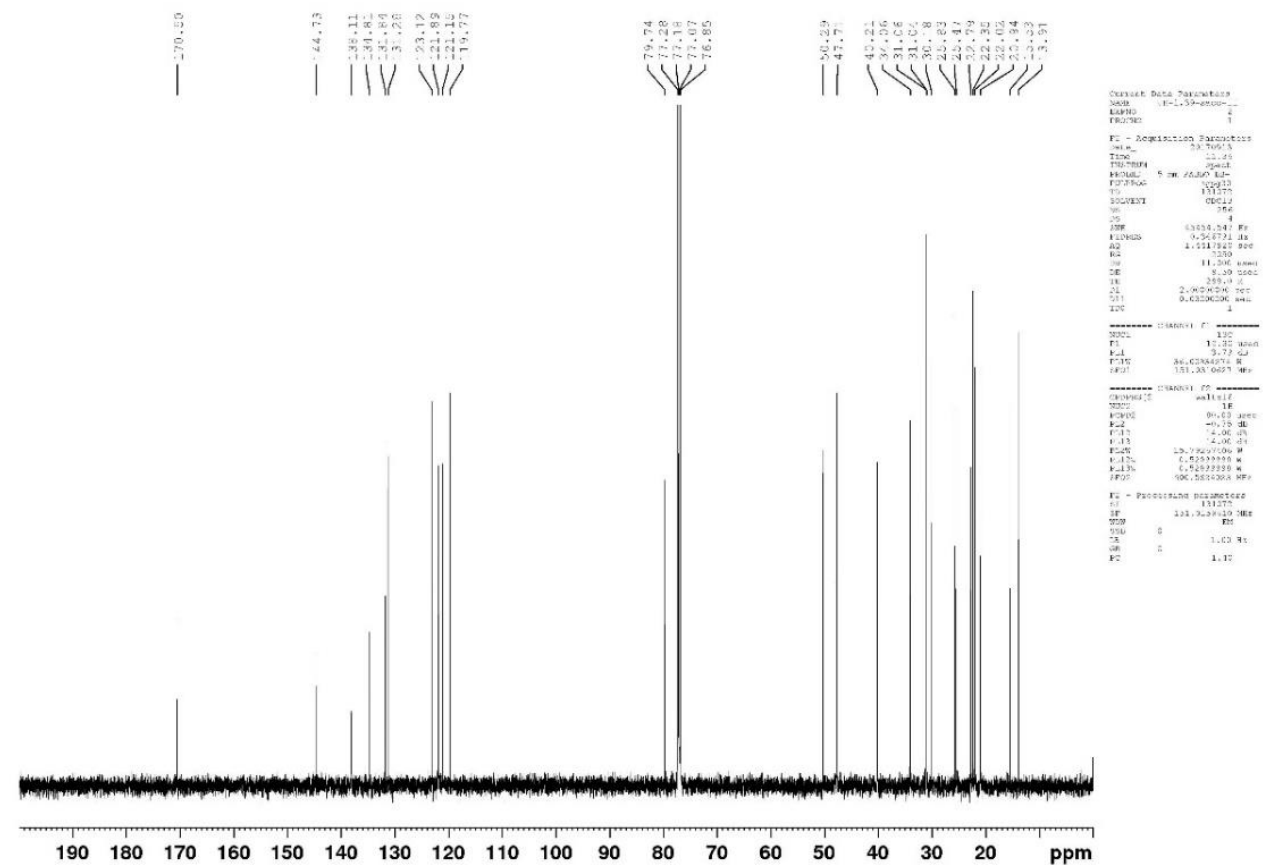

Figure S40.

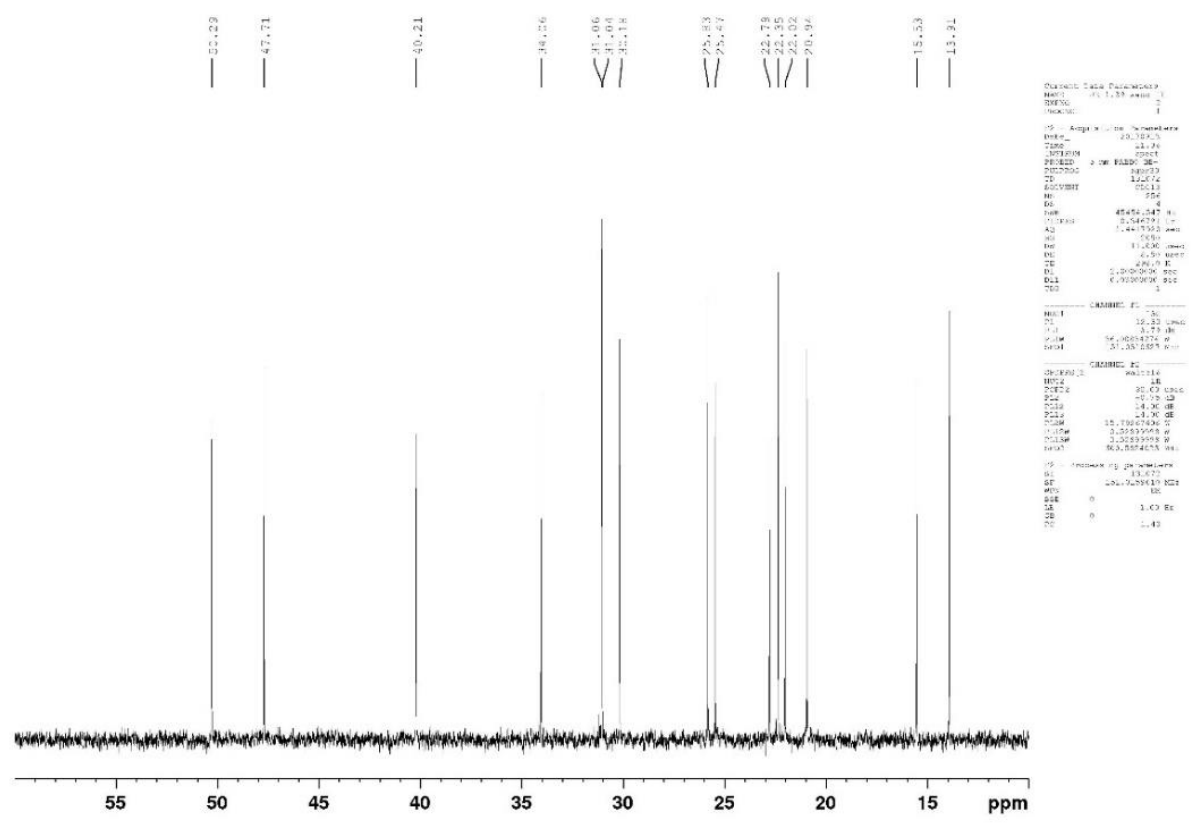

Figure S41. 
3-Heptyl-1-[(1R.2S.5R)-(-)-menthoxymethyl]imidazolium saccharinate $\left[\mathrm{C}_{7}-\mathrm{Im}-\mathrm{CH}_{2} \mathrm{O}\right.$ Men][Sacc] (3g)<smiles>CCn1cc[n+](CO[C@H]2C[C@H](C)CC[C@H]2C(C)C)c1</smiles>
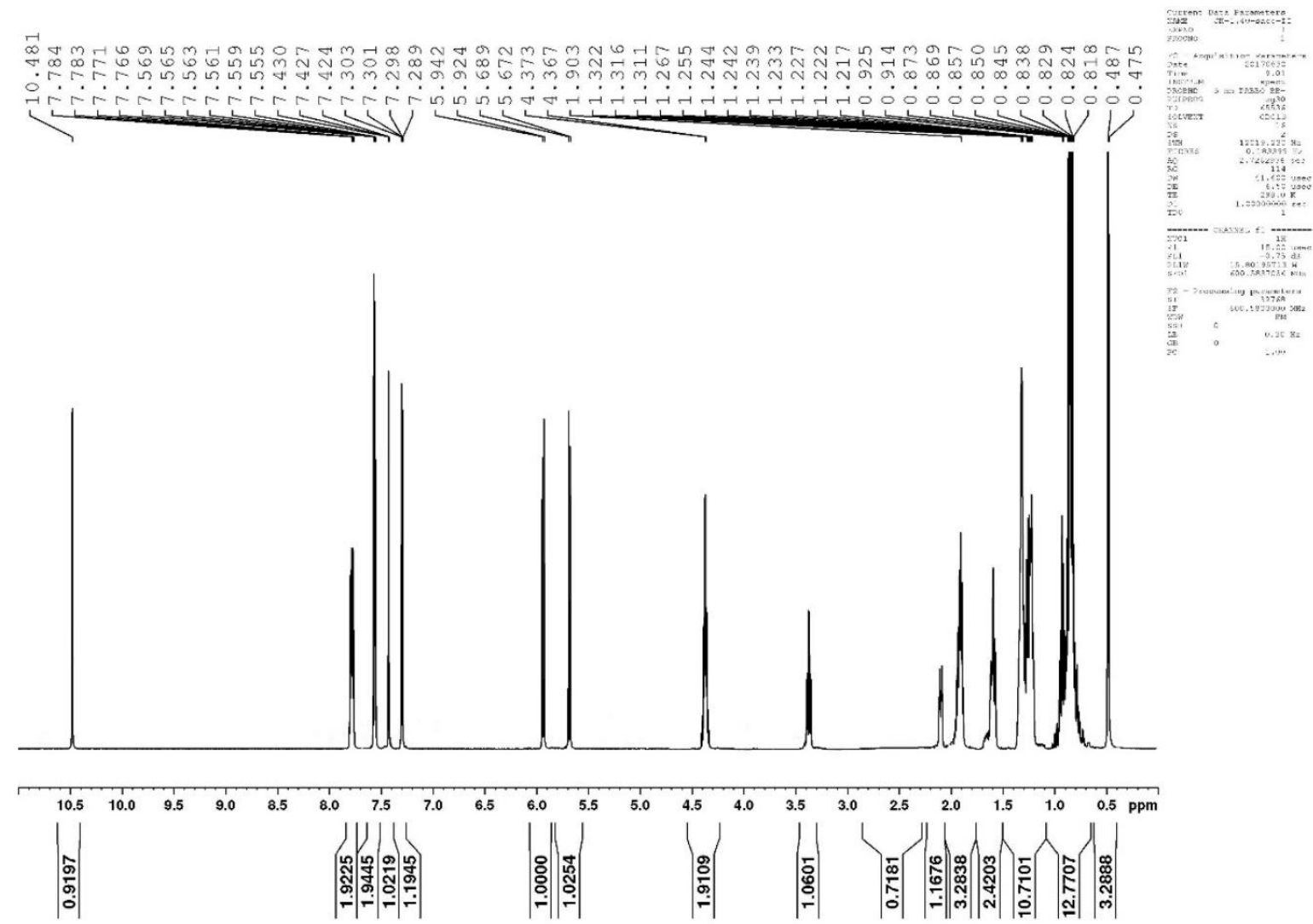

Figure S42. 


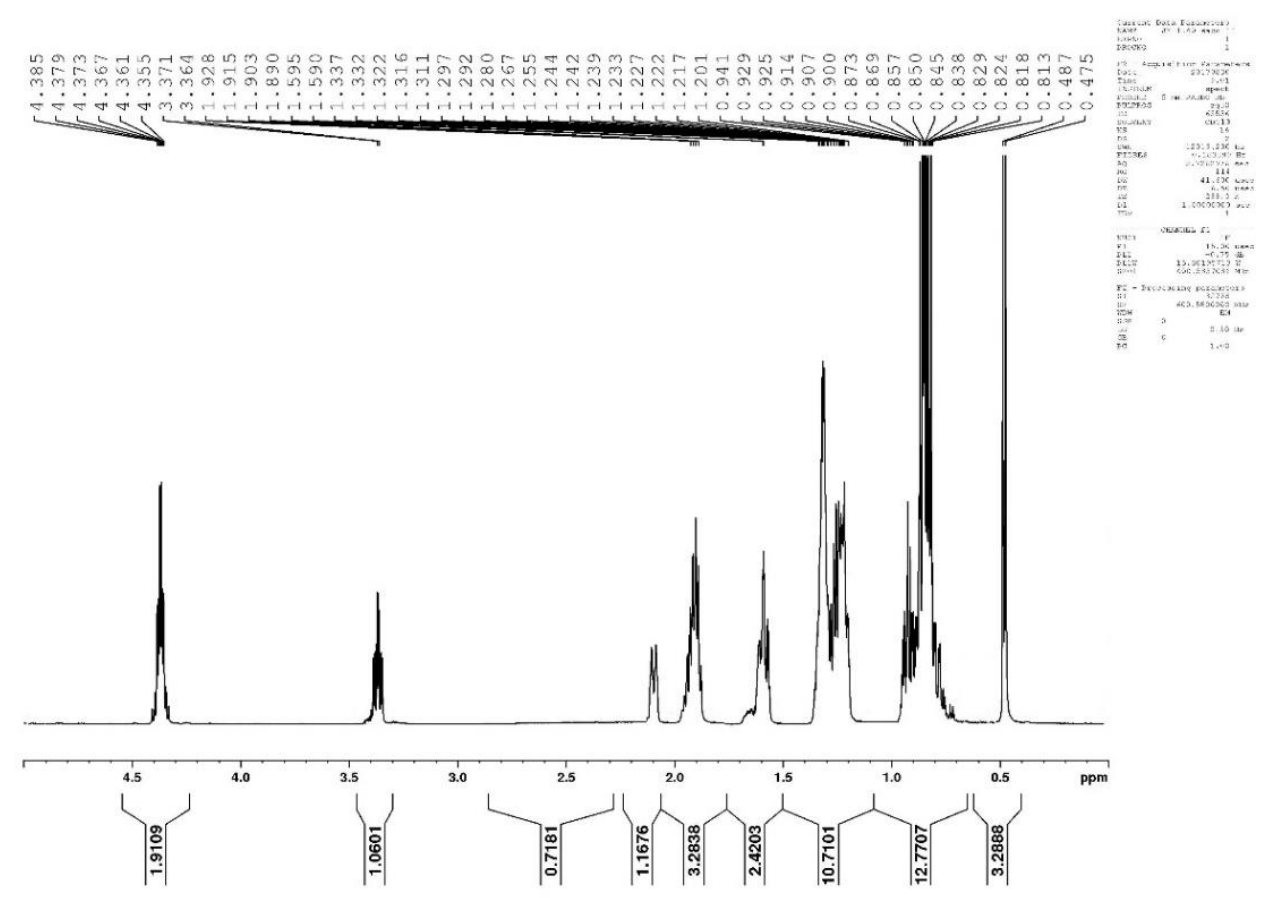

Figure S43.

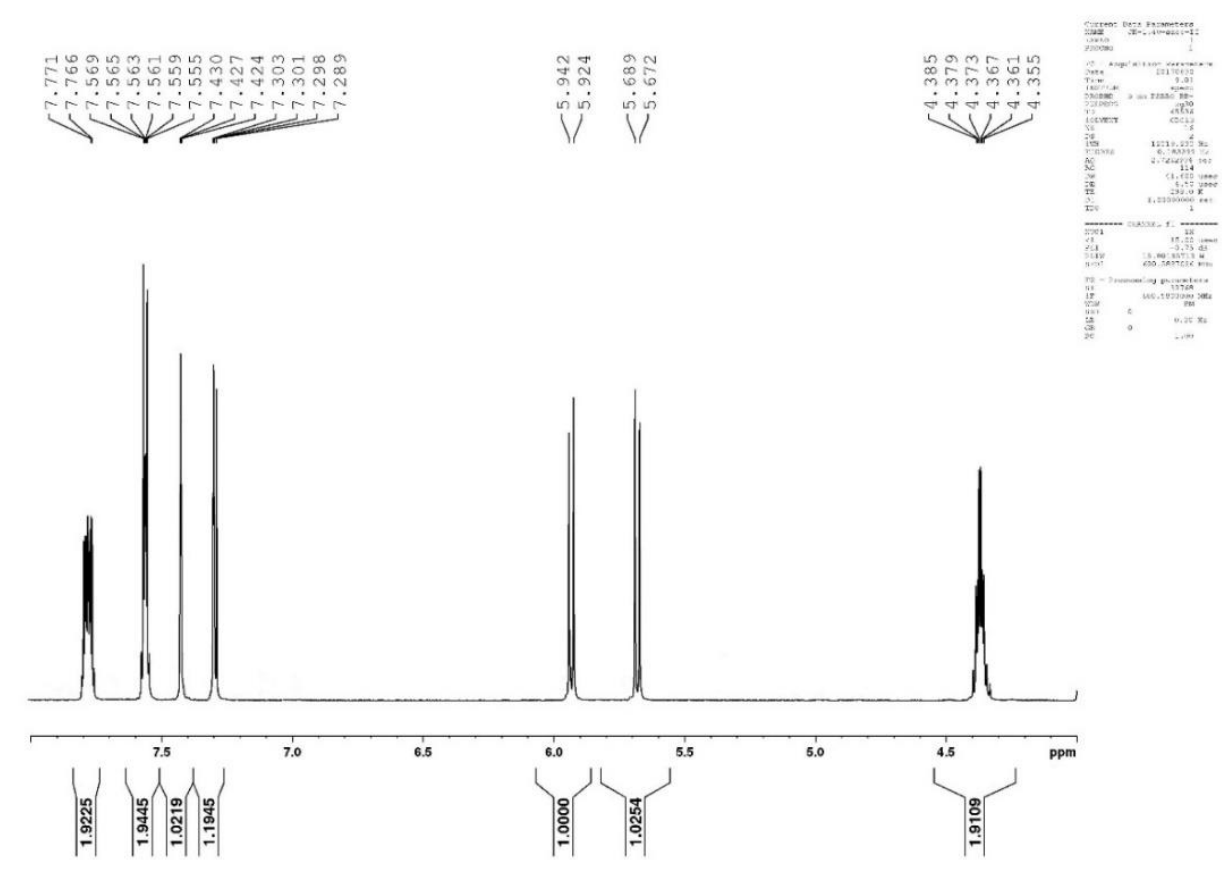

Figure S44. 


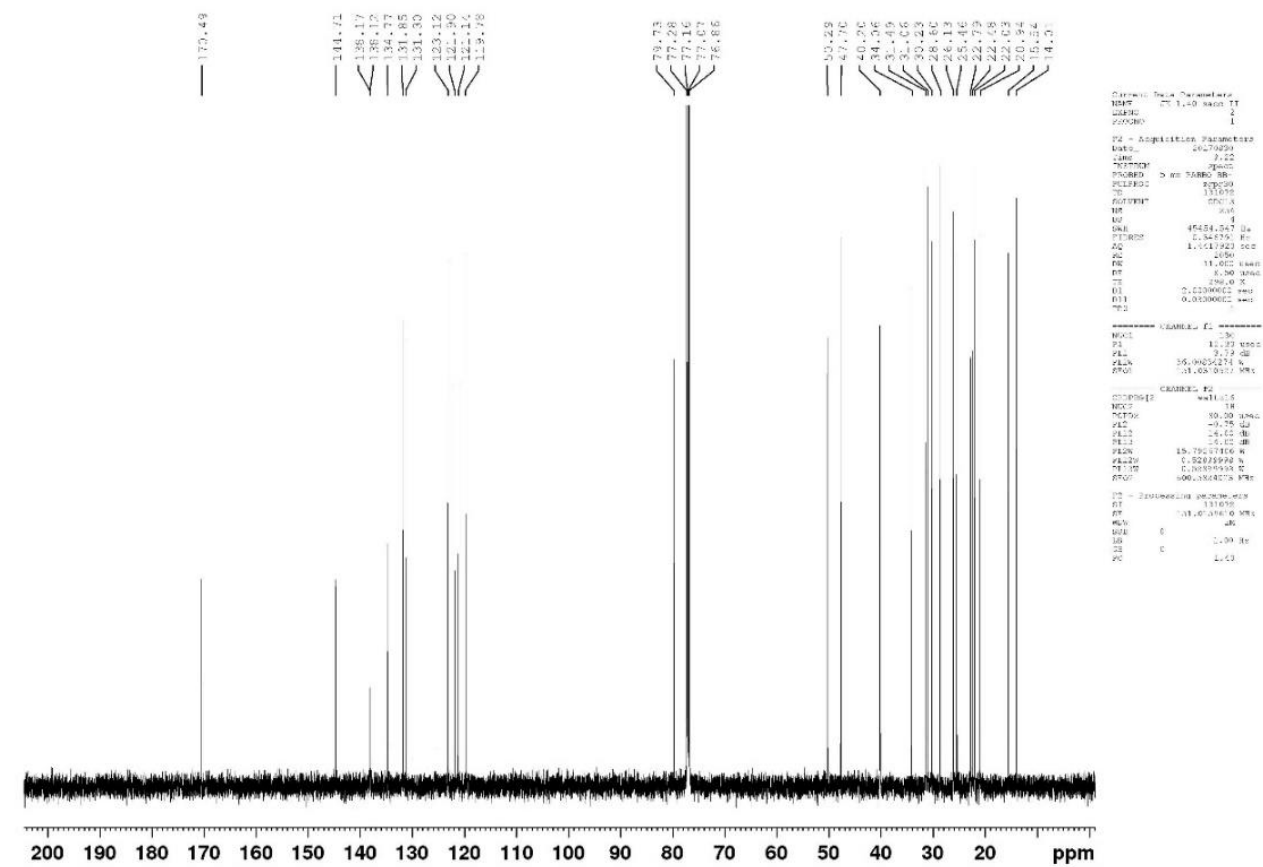

Figure S45.

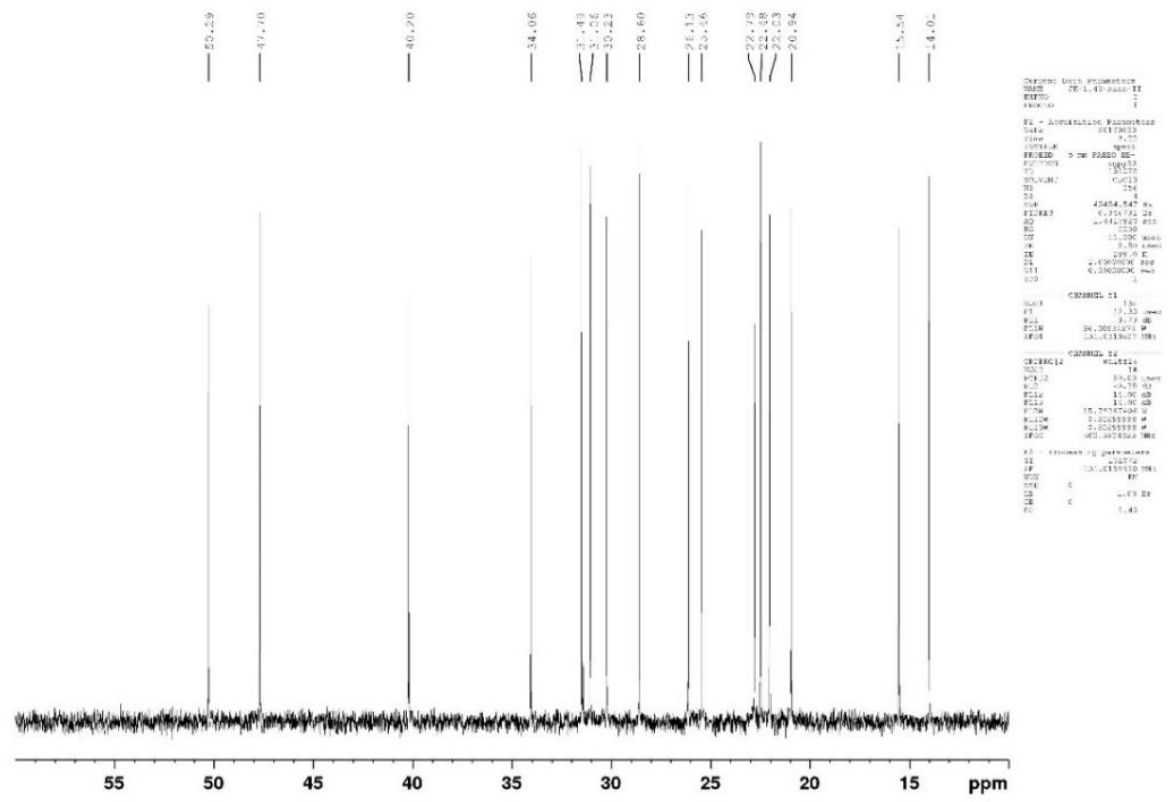

Figure S46. 


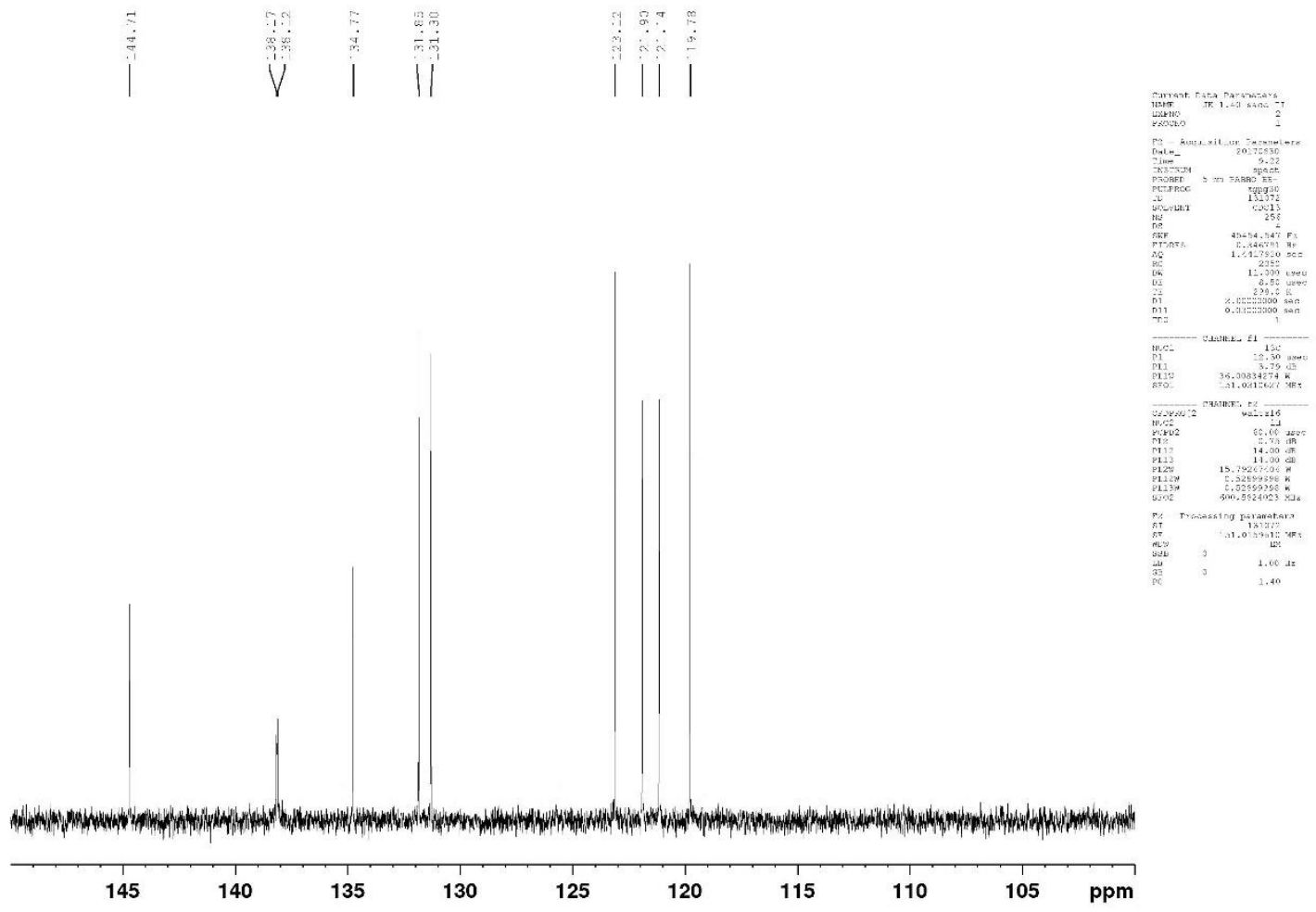

Figure S47. 


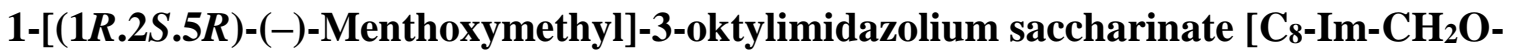
Men][Sacc] (3h)<smiles>CCCC[n+]1ccn(CO[C@H]2C[C@H](C)CC[C@H]2C(C)C)c1</smiles>

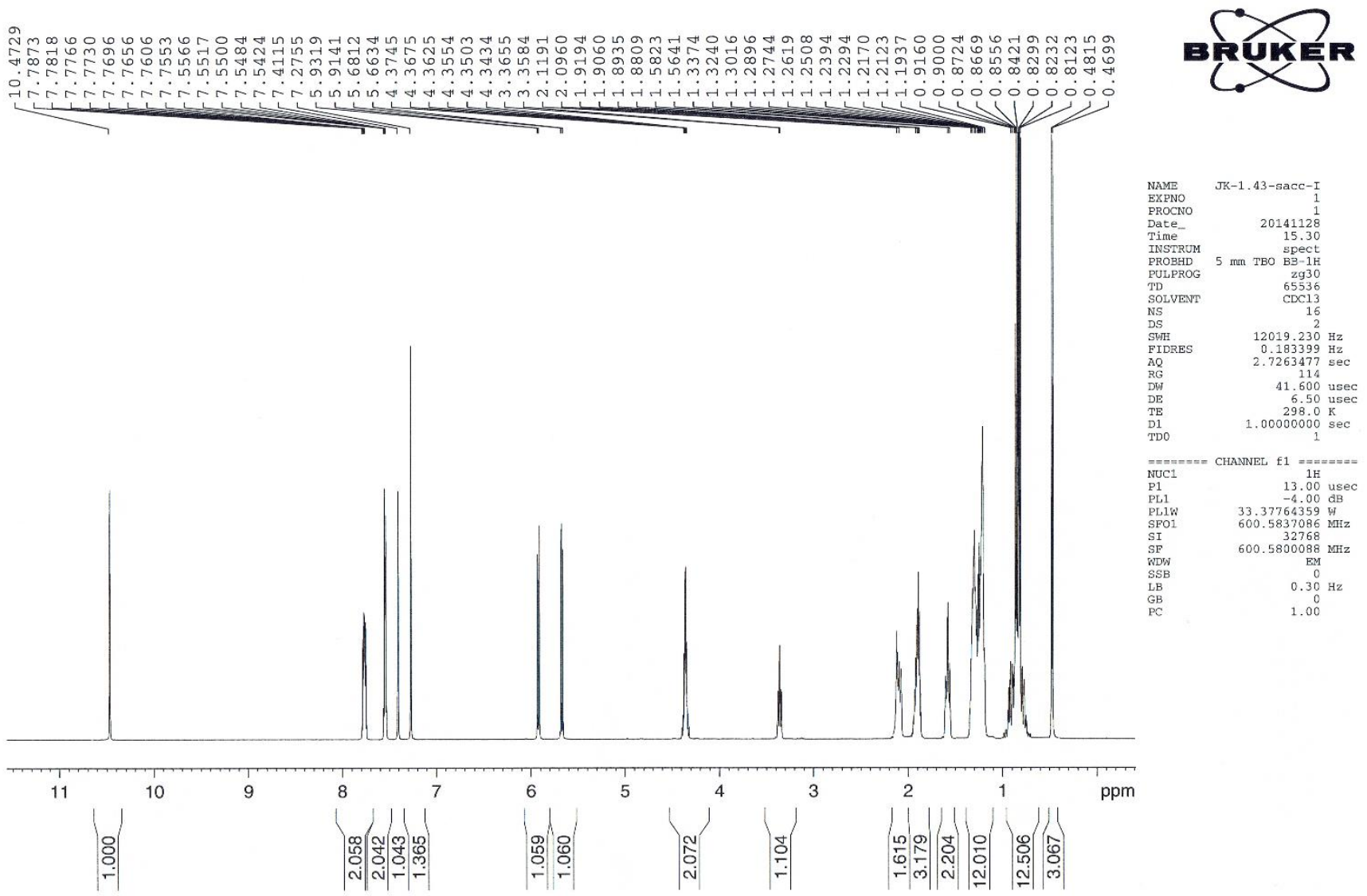

Figure S48. 


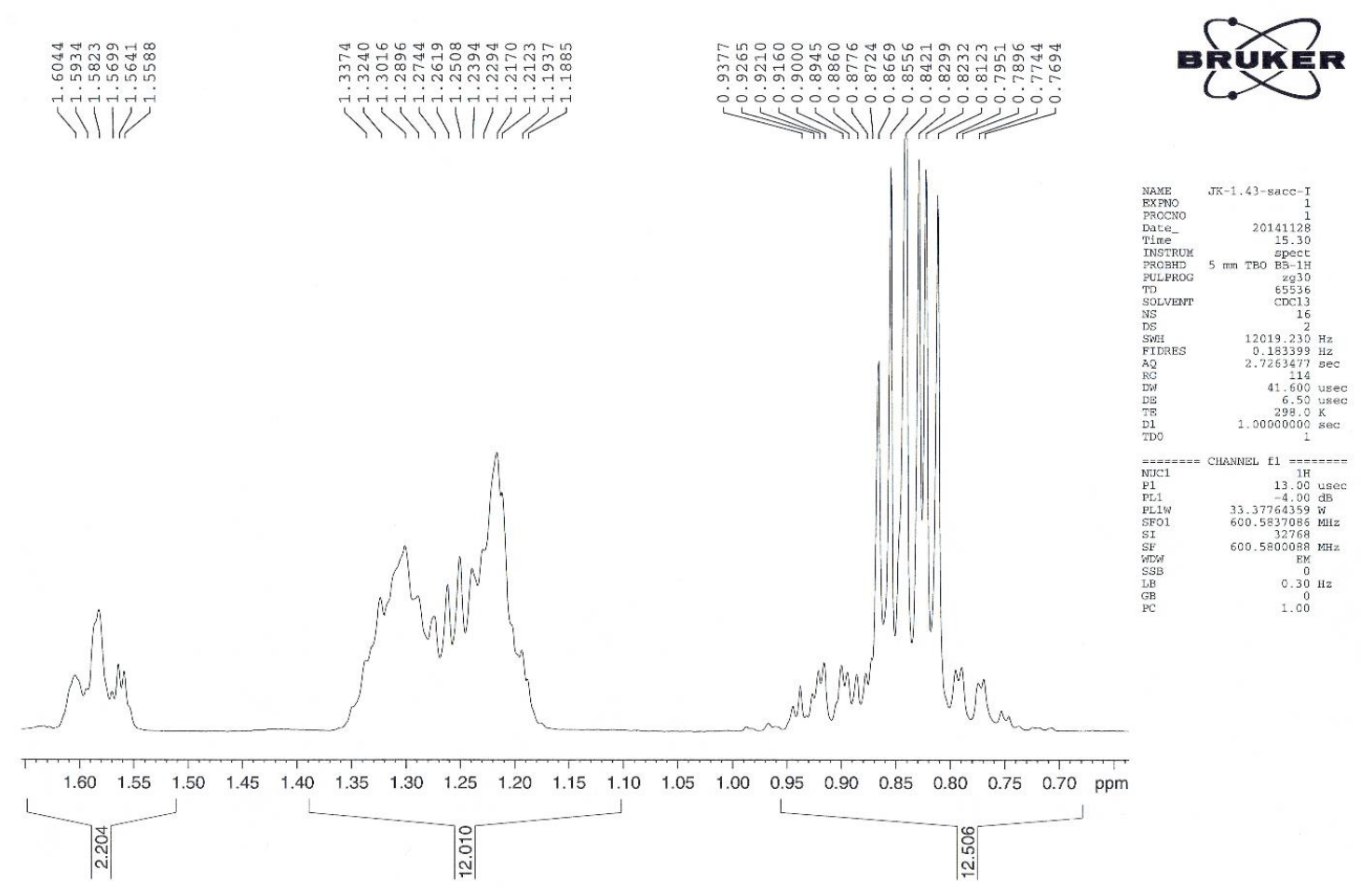

Figure S49.

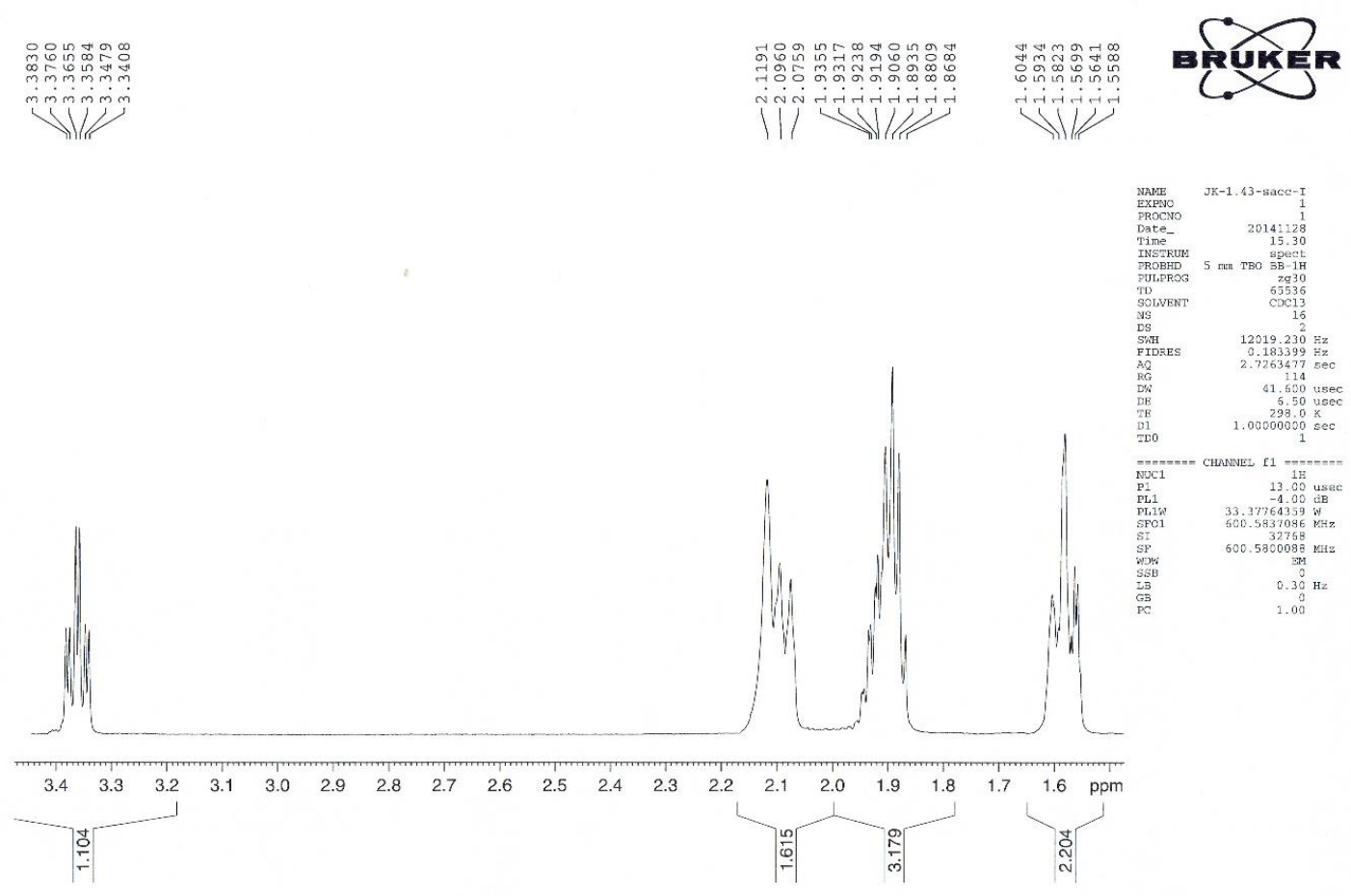

Figure S50. 


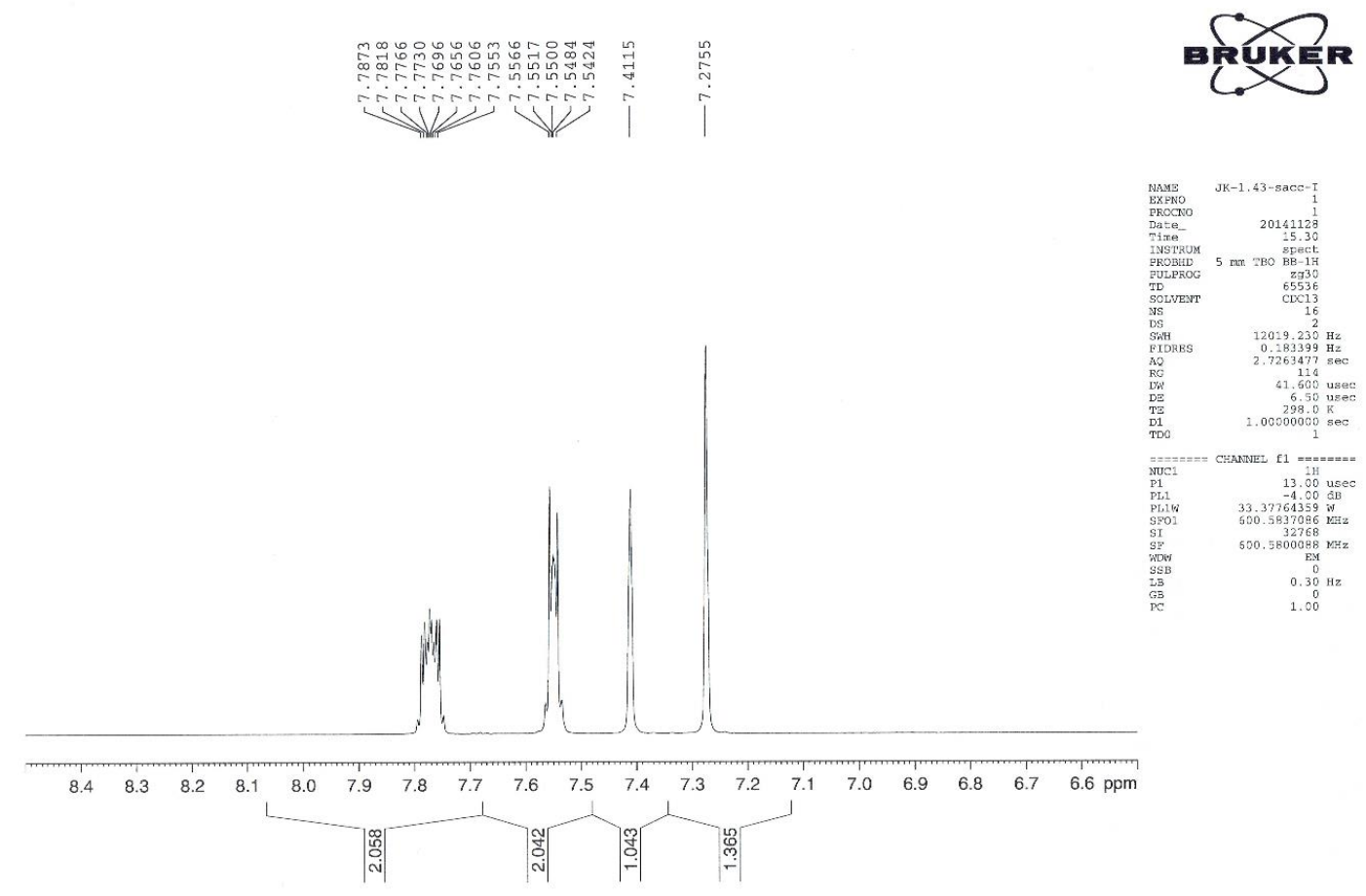

Figure S51.
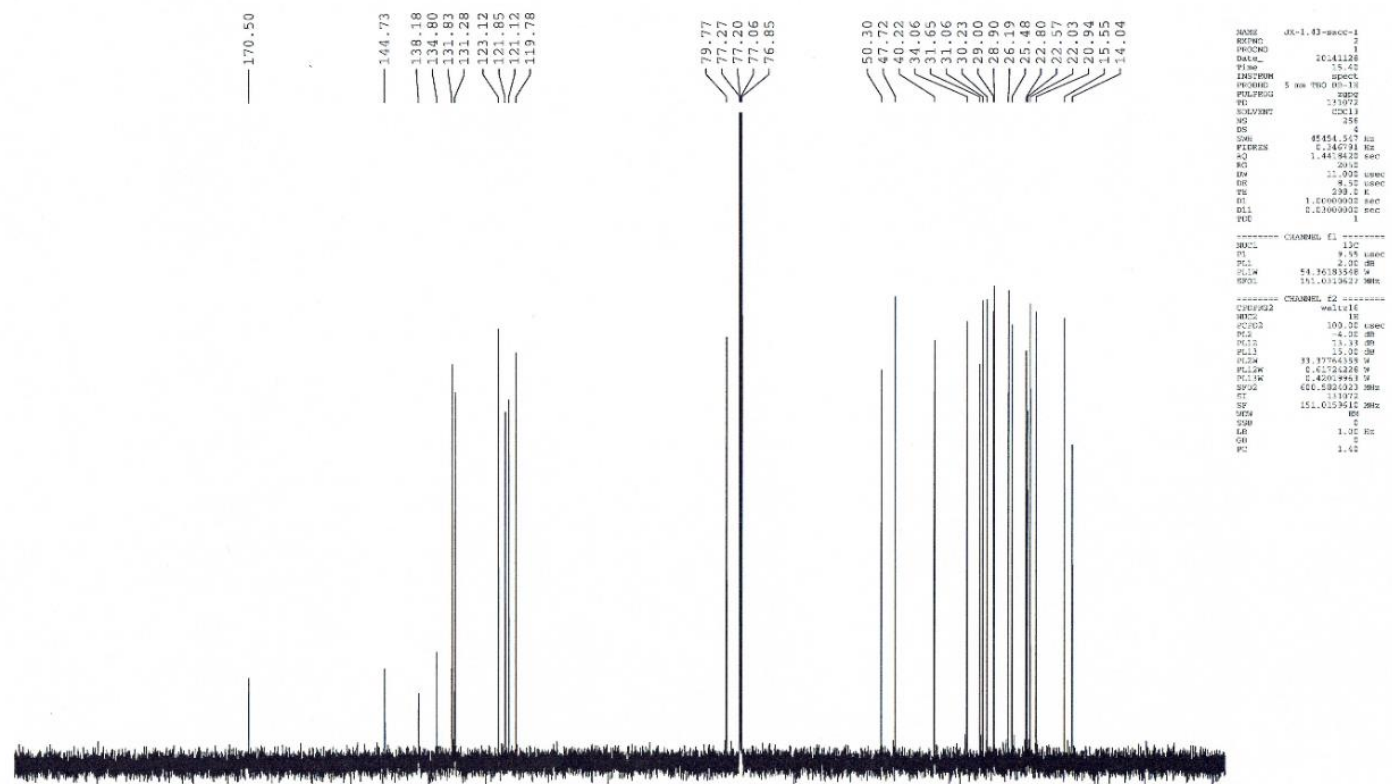

$\begin{array}{lllllllllllllllllllllll}210 & 200 & 190 & 180 & 170 & 160 & 150 & 140 & 130 & 120 & 110 & 100 & 90 & 80 & 70 & 60 & 50 & 40 & 30 & 20 & 10 & 0 & \mathrm{ppm}\end{array}$

Figure S52. 


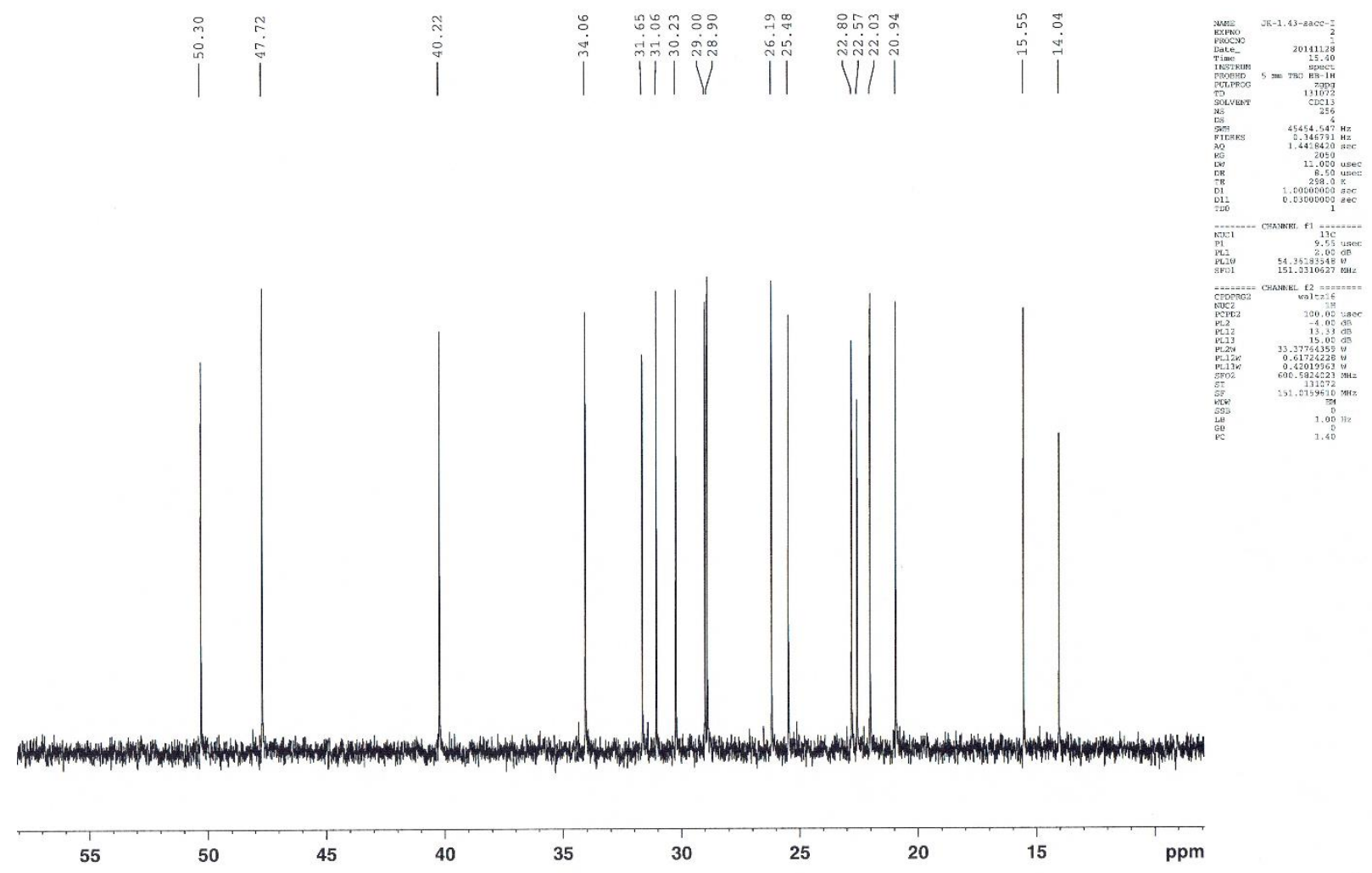

Figure S53. 
1-[(1R.2S.5R)-(-)-Menthoxymethyl]-3-nonylimidazolium saccharinate [ $\mathrm{C}_{9}-\mathrm{Im}-\mathrm{CH}_{2} \mathrm{O}$ Men][Sacc] (3i)<smiles></smiles>

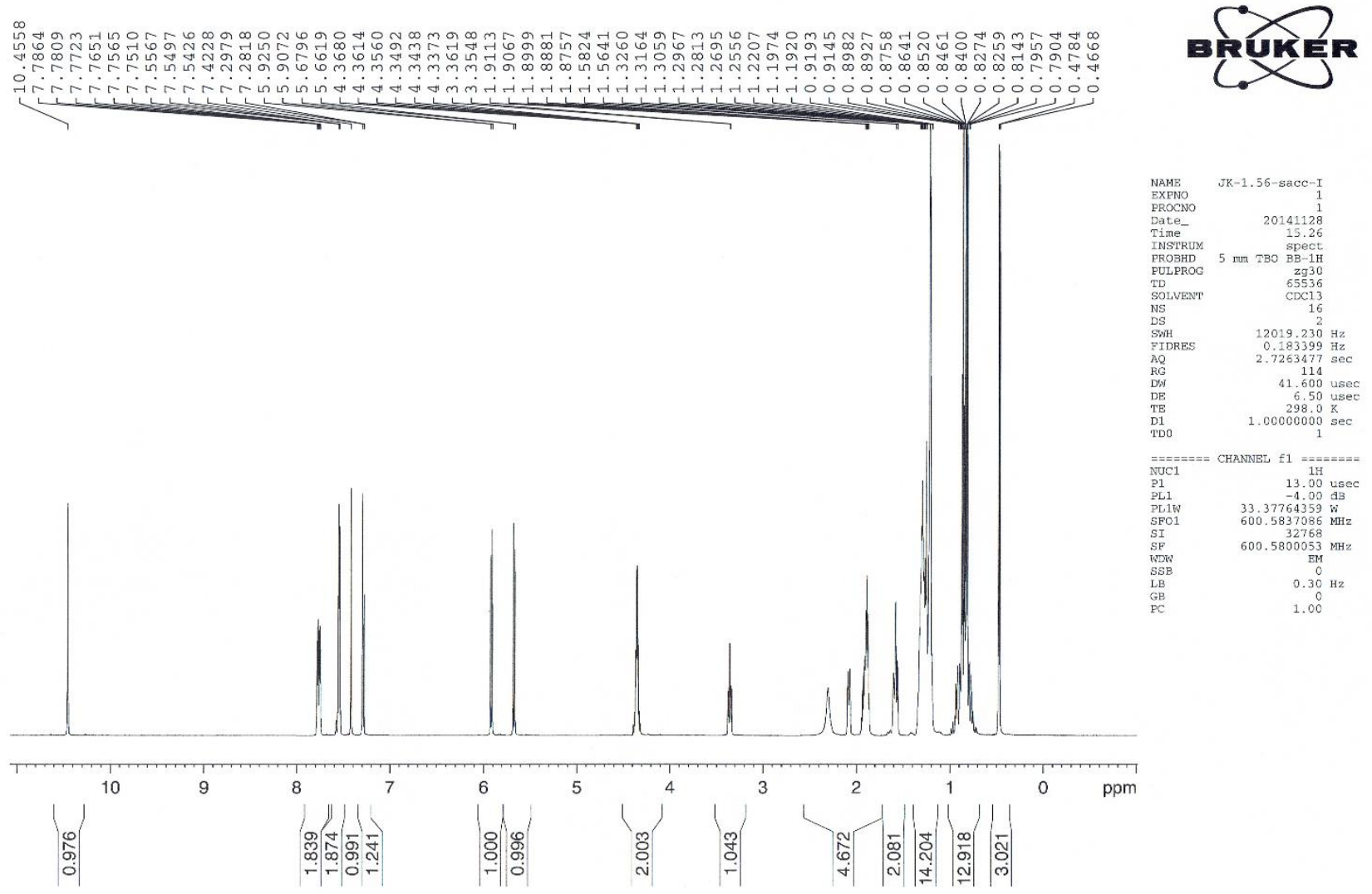

Figure S54. 


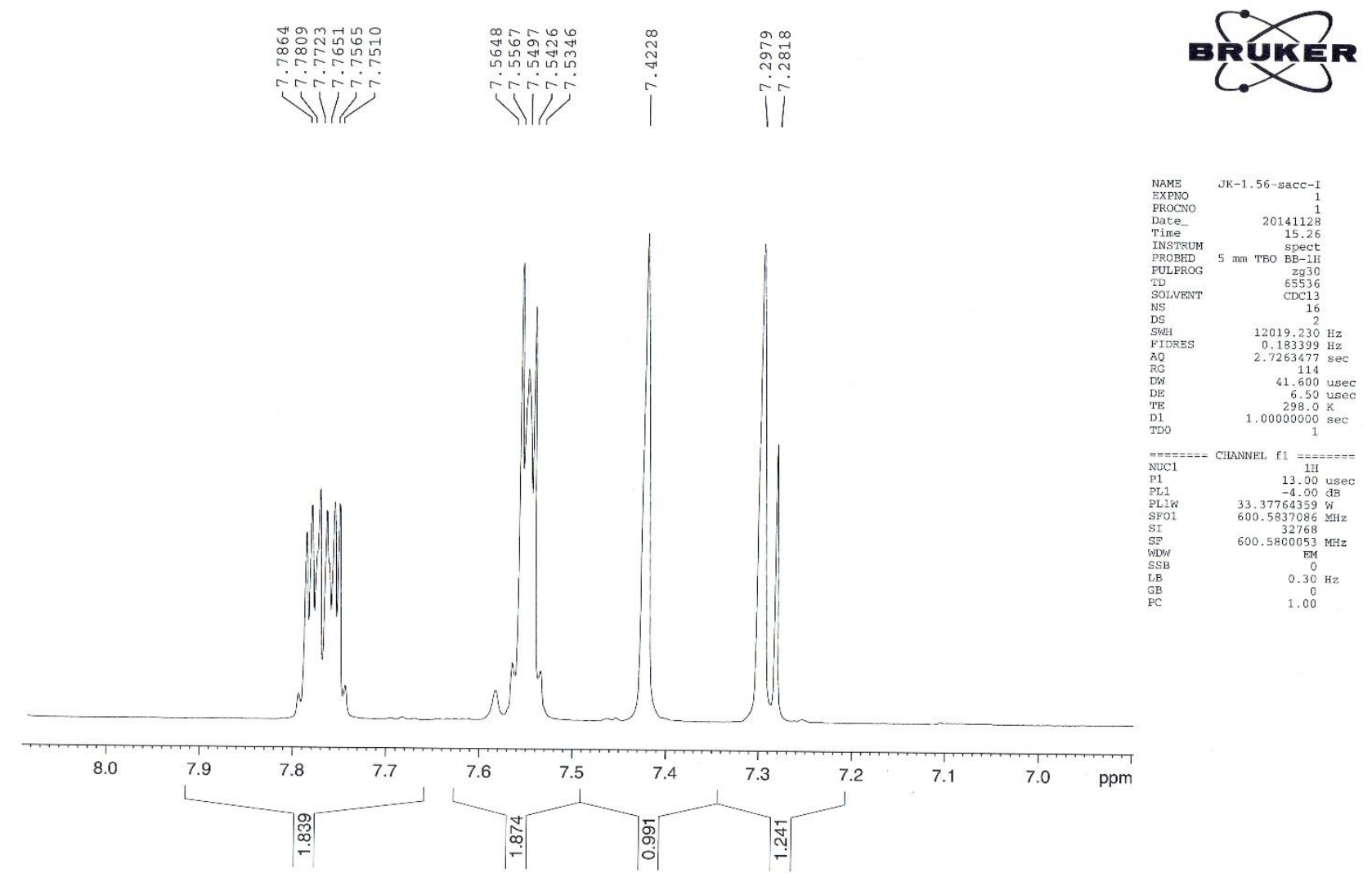

Figure S55. 


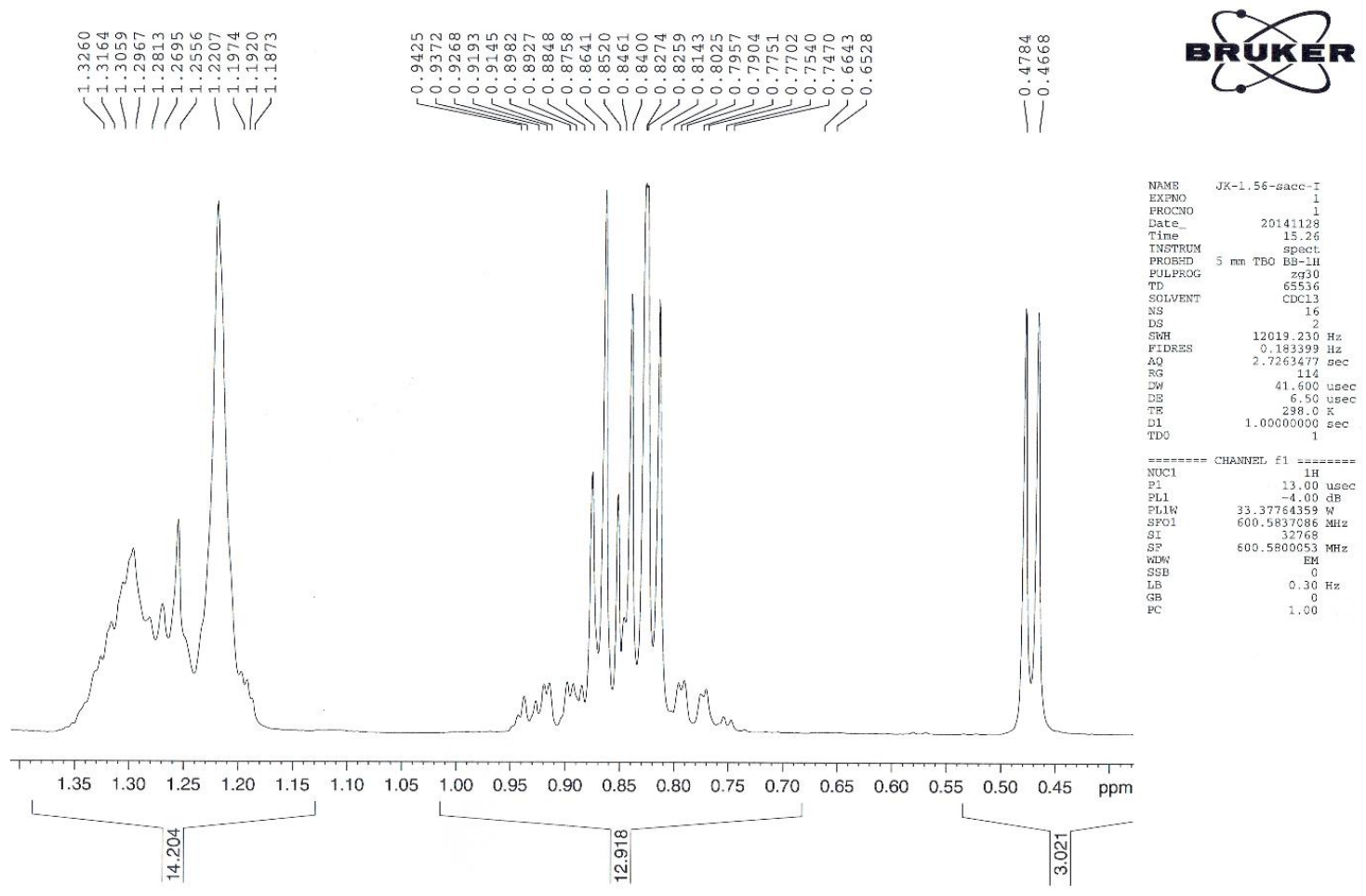

Figure S56.

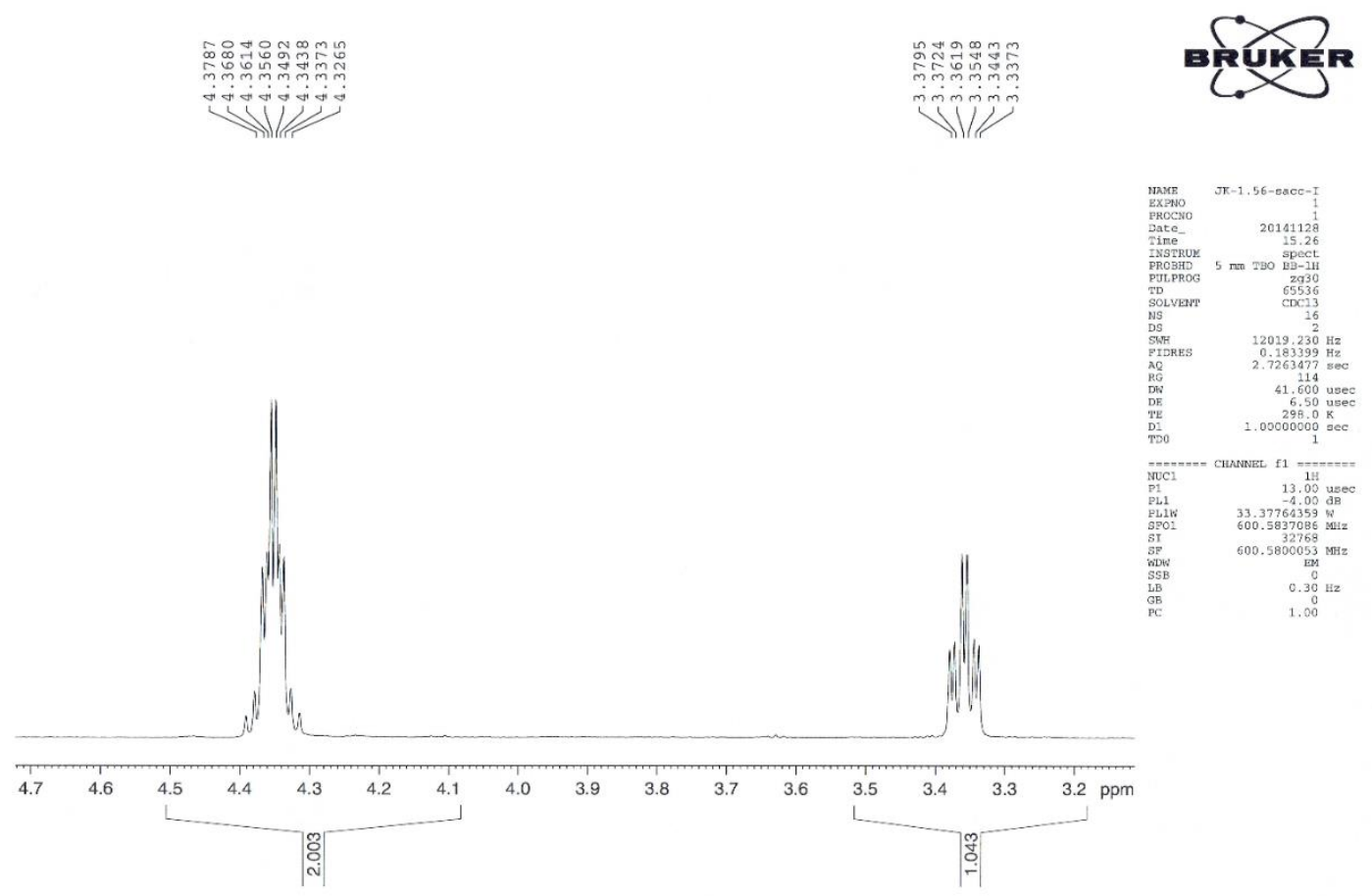

Figure S57. 


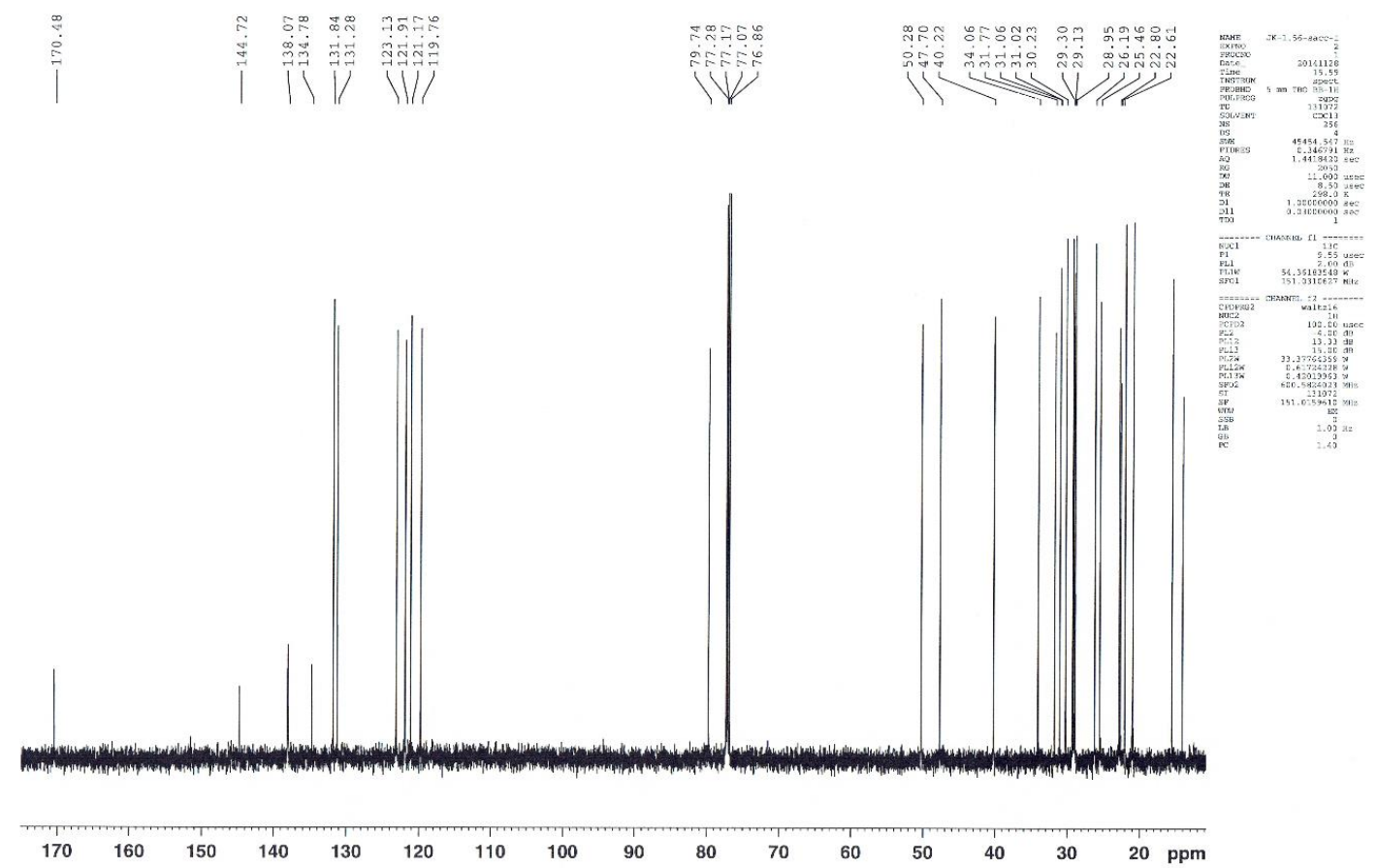

Figure S58.

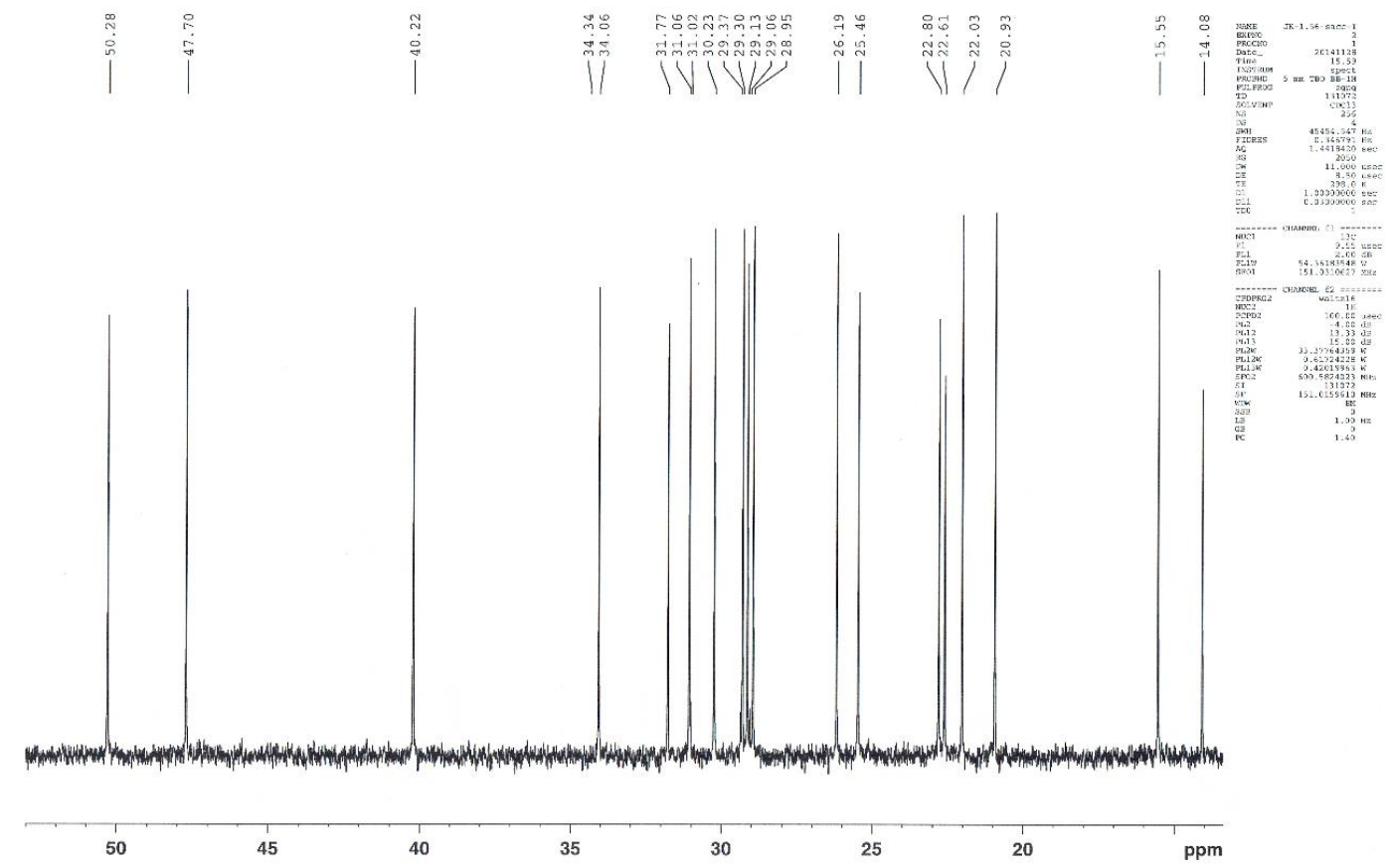

Figure S59. 
3-Decyl-1-[(1R.2S.5R)-(-)-menthoxymethyl]imidazolium saccharinate $\left[\mathrm{C}_{10}-\mathrm{Im}-\mathrm{CH}_{2} \mathrm{O}\right.$ Men][Sacc] (3j)

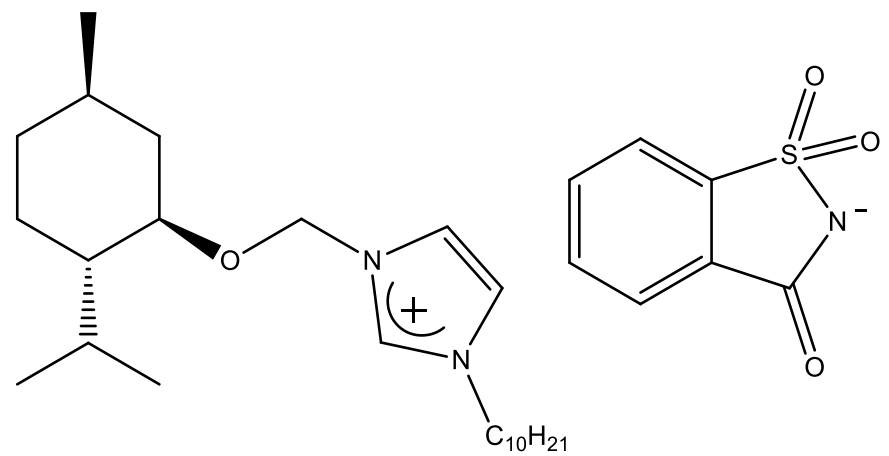
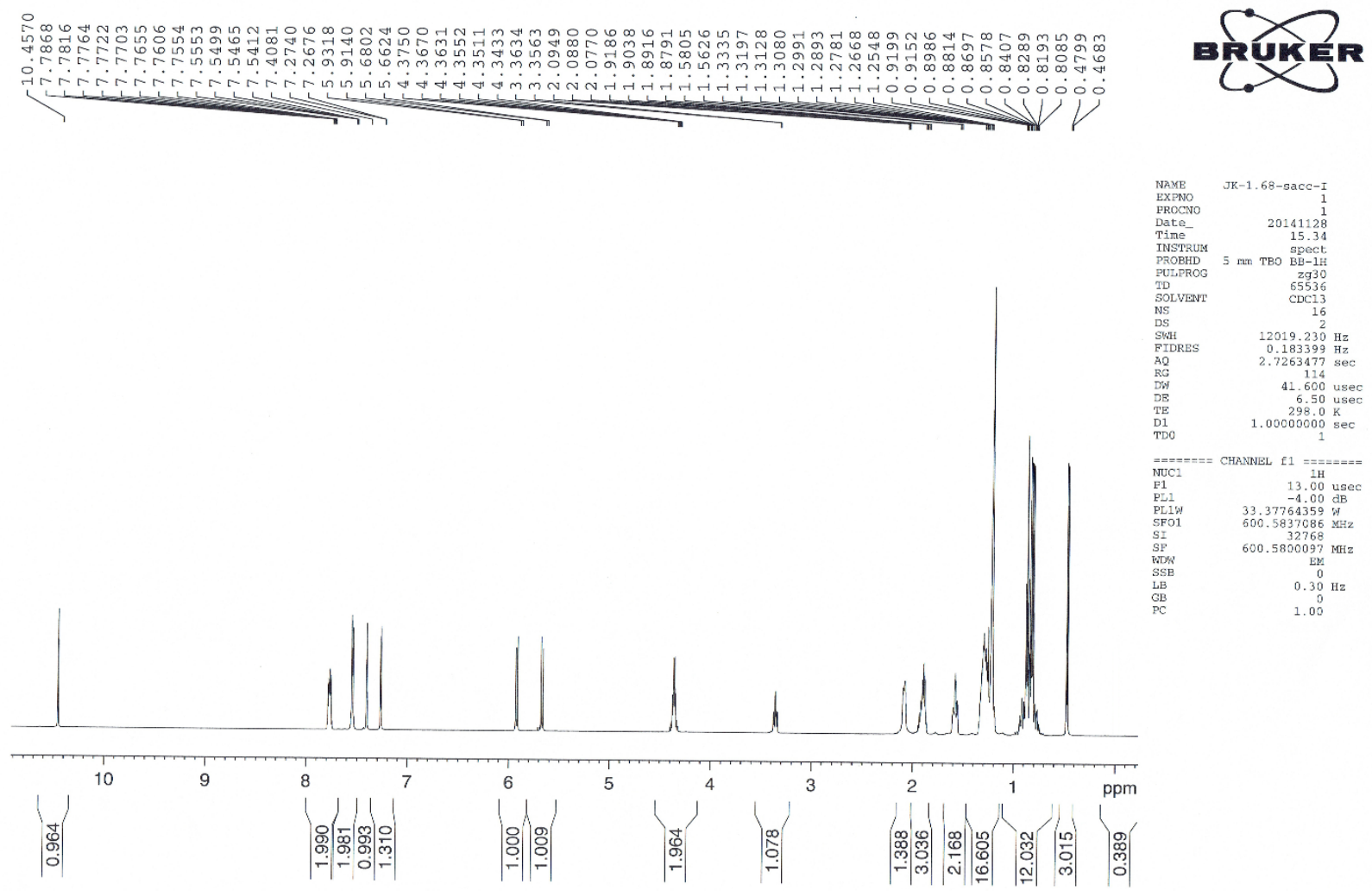

Figure S60. 


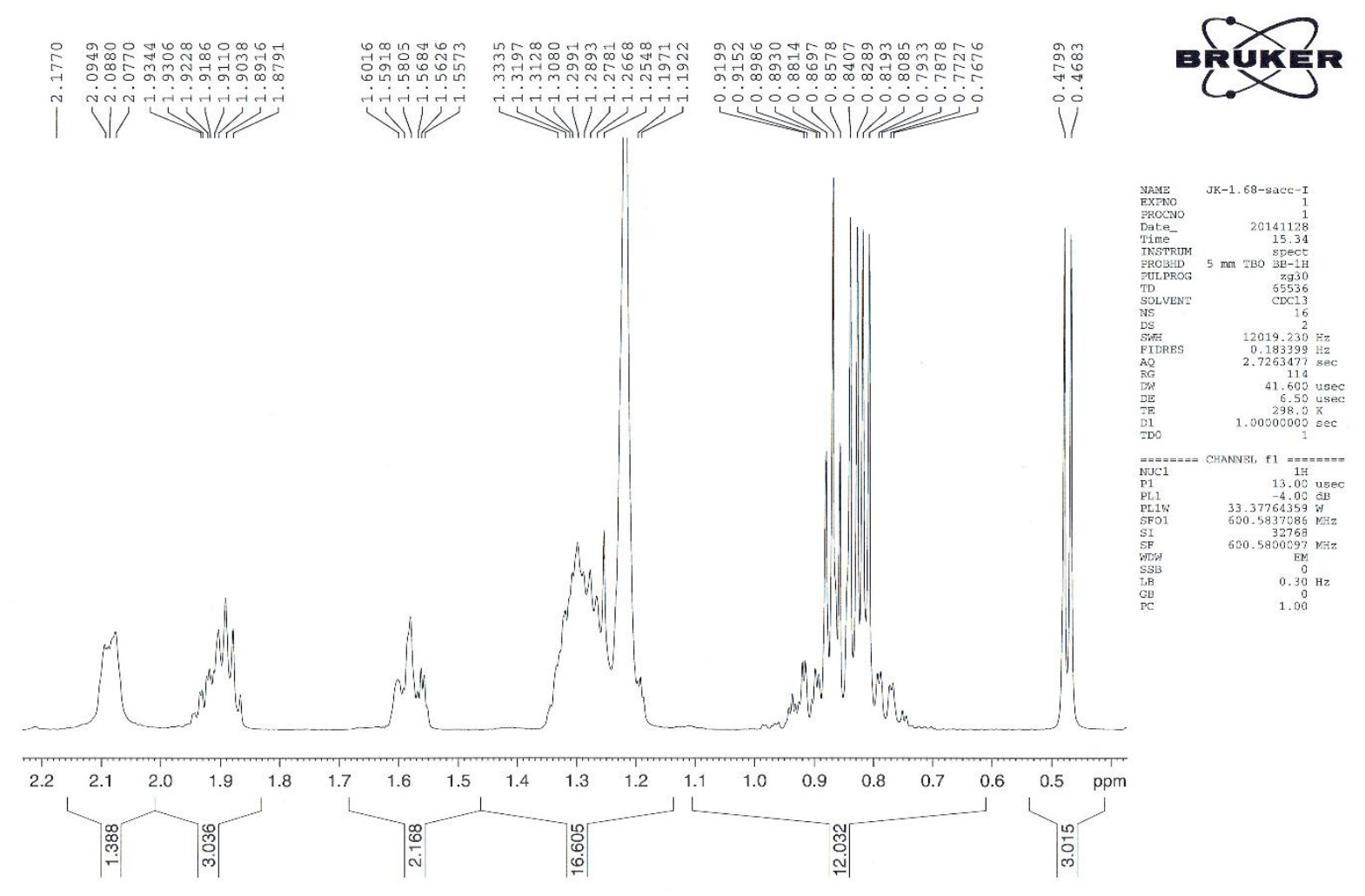

Figure S61.

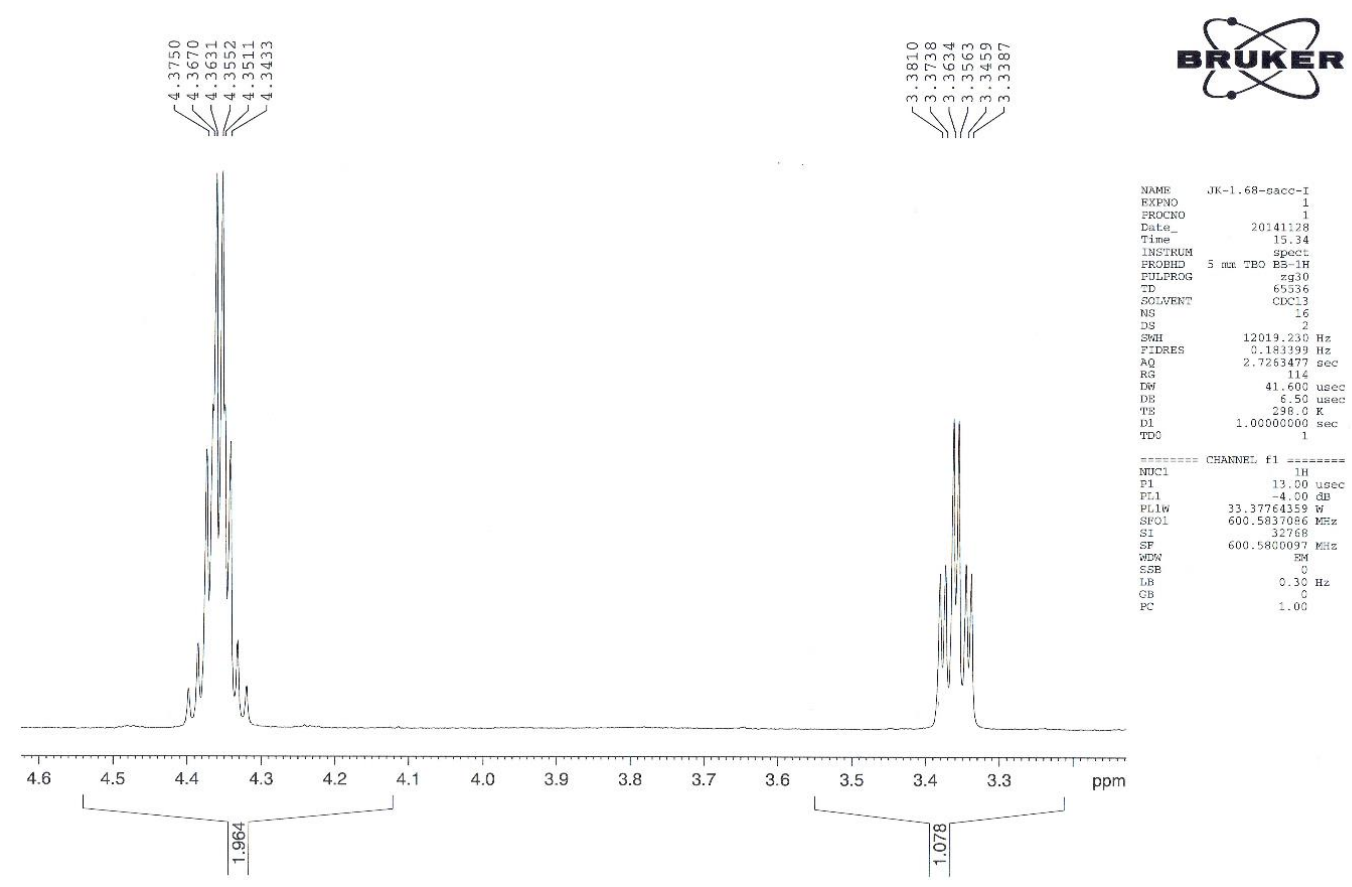

Figure S62. 

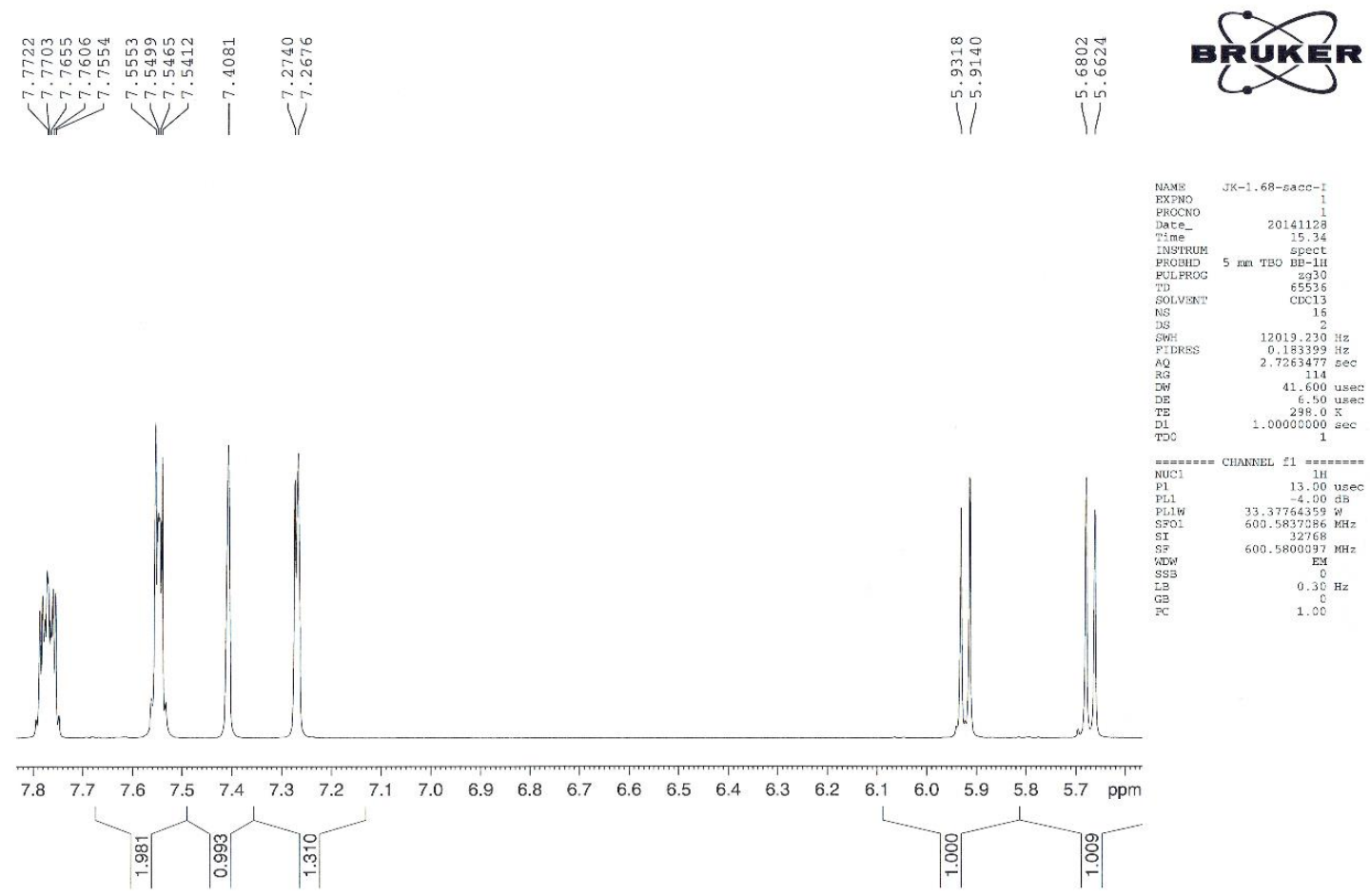

Figure S63.

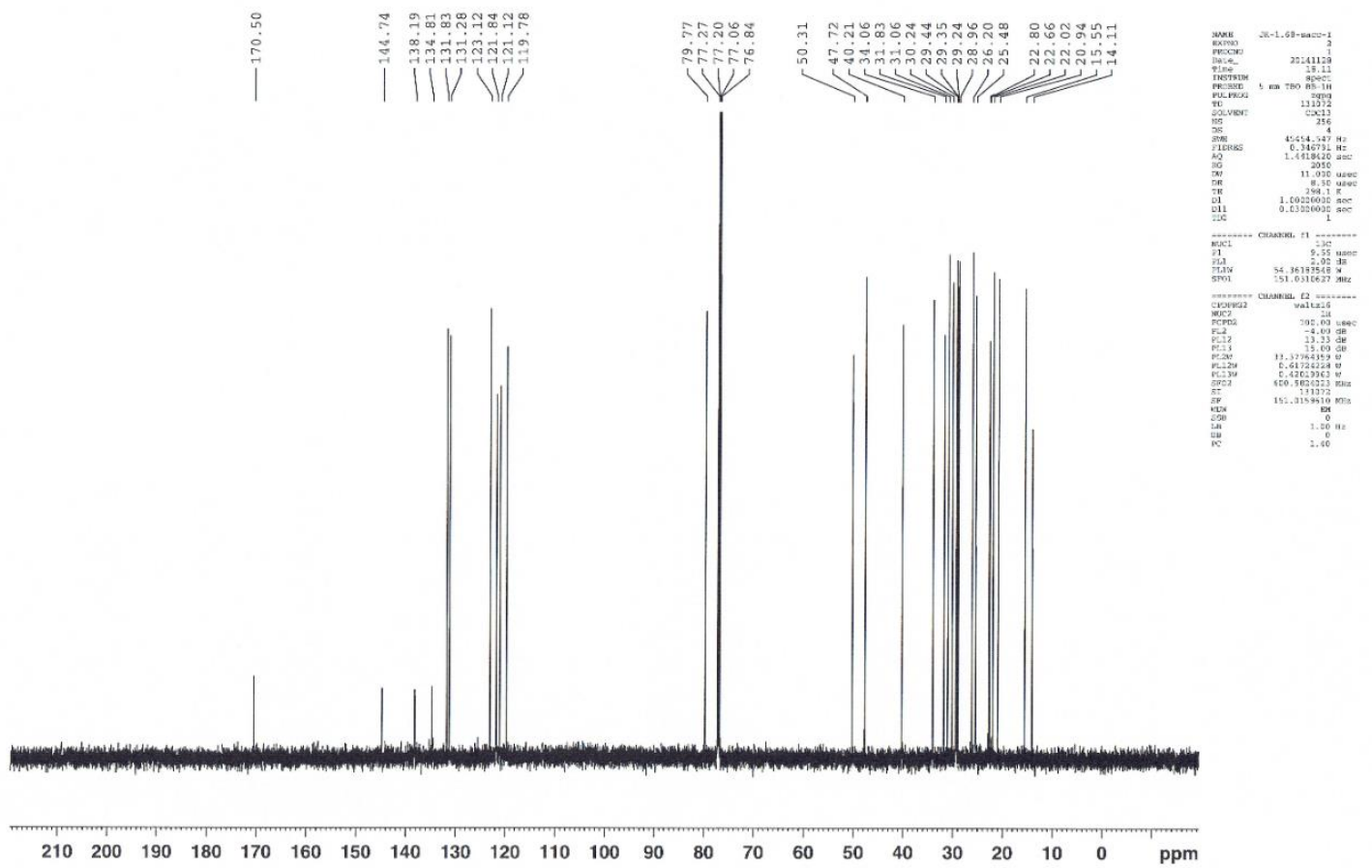

Figure S64. 


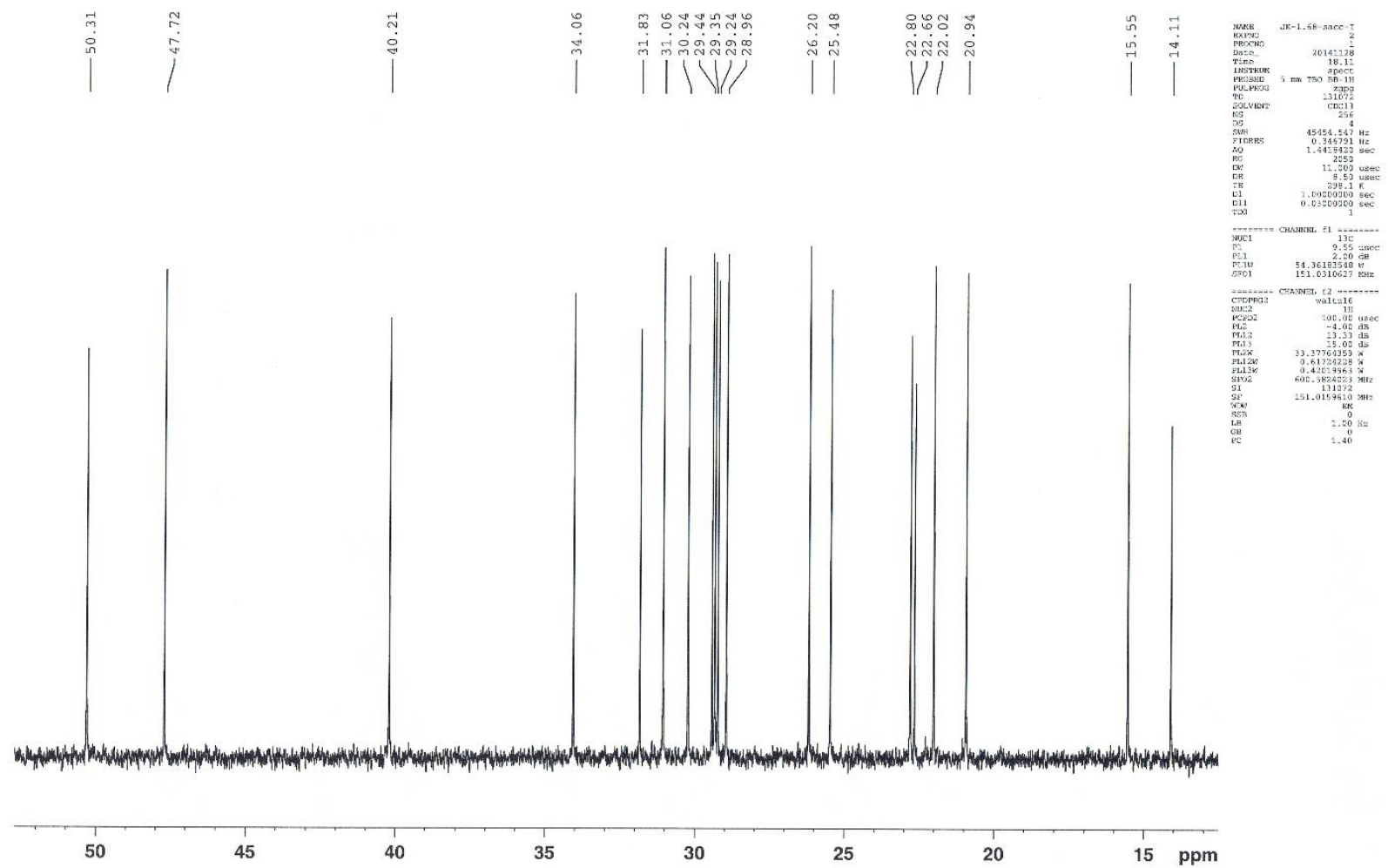

Figure S65. 
1-[(1R.2S.5R)-(-)-Menthoxymethyl]-3-undecylimidazolium saccharinate [ $\mathrm{C}_{11}-\mathrm{Im}-\mathrm{CH}_{2} \mathrm{O}$ Men][Sacc] (3k)

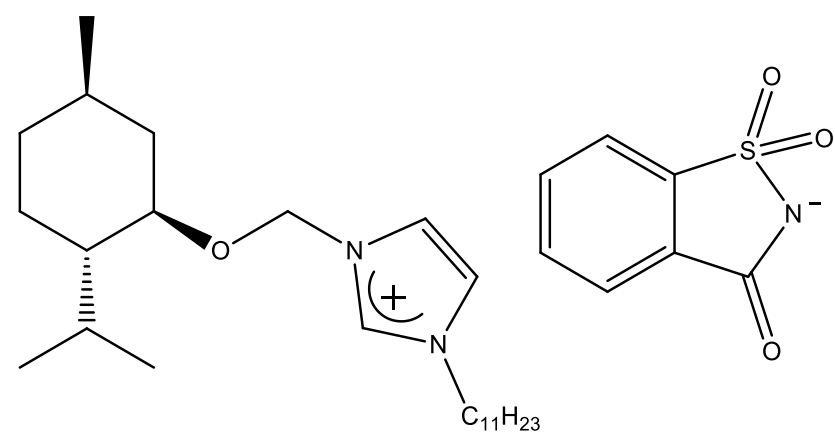

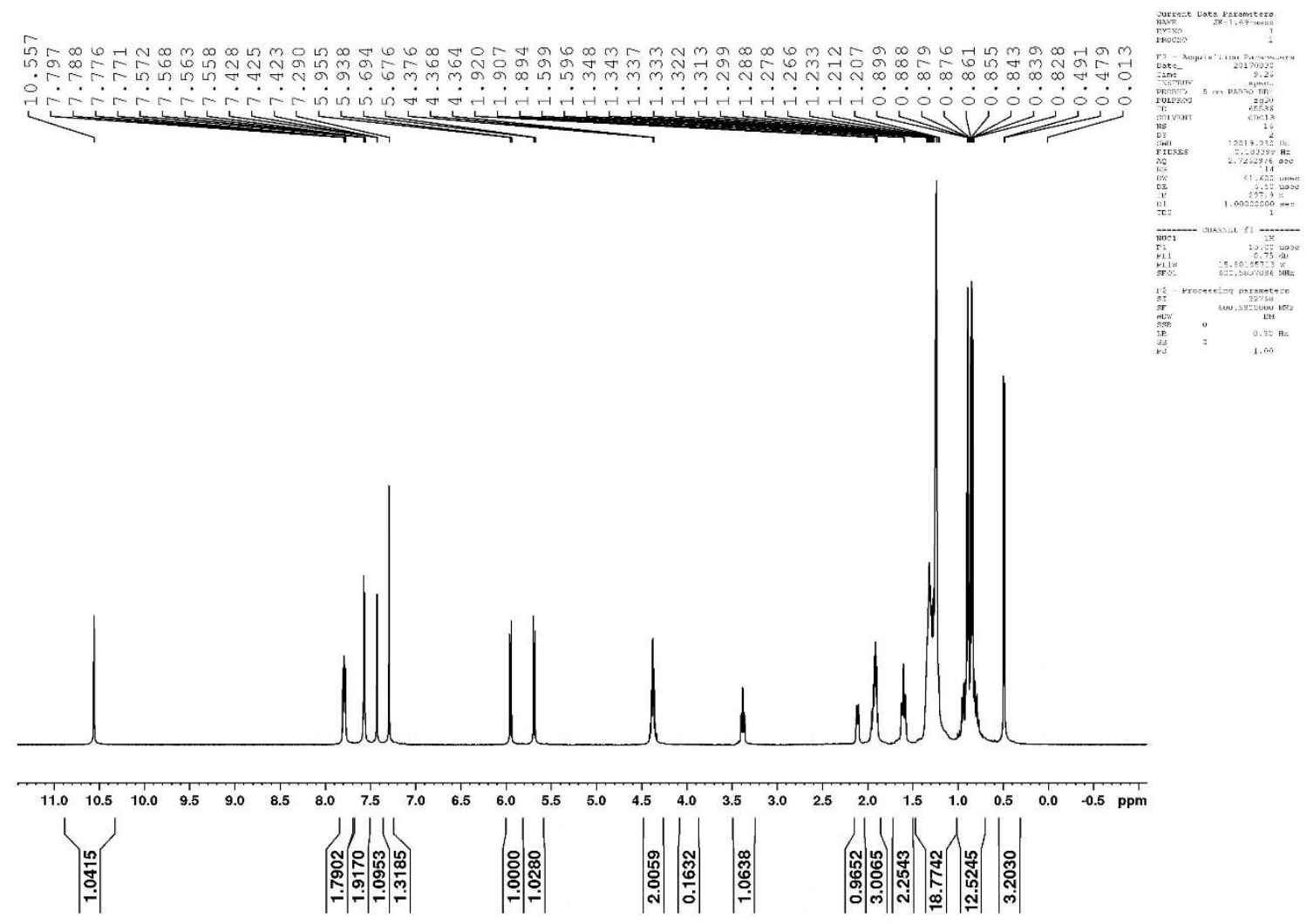

Figure S66. 


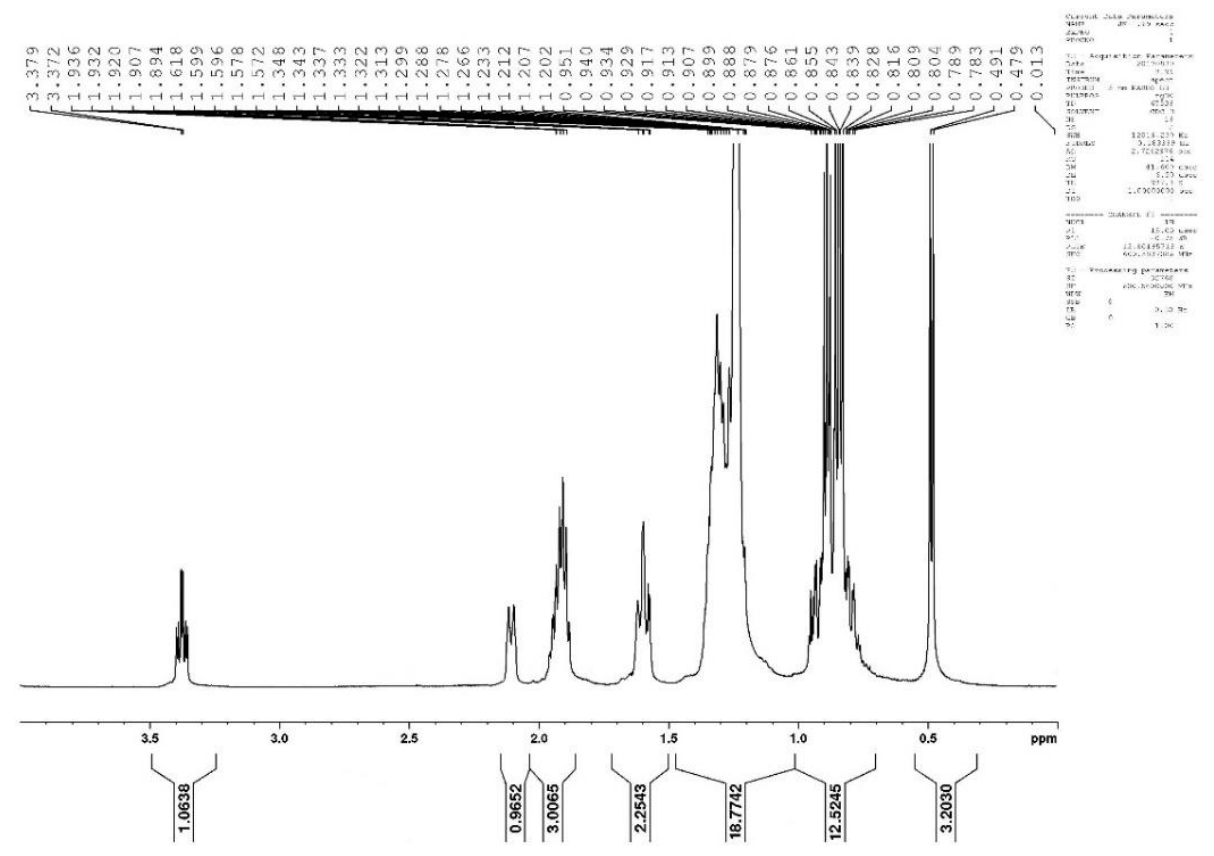

Figure S67.

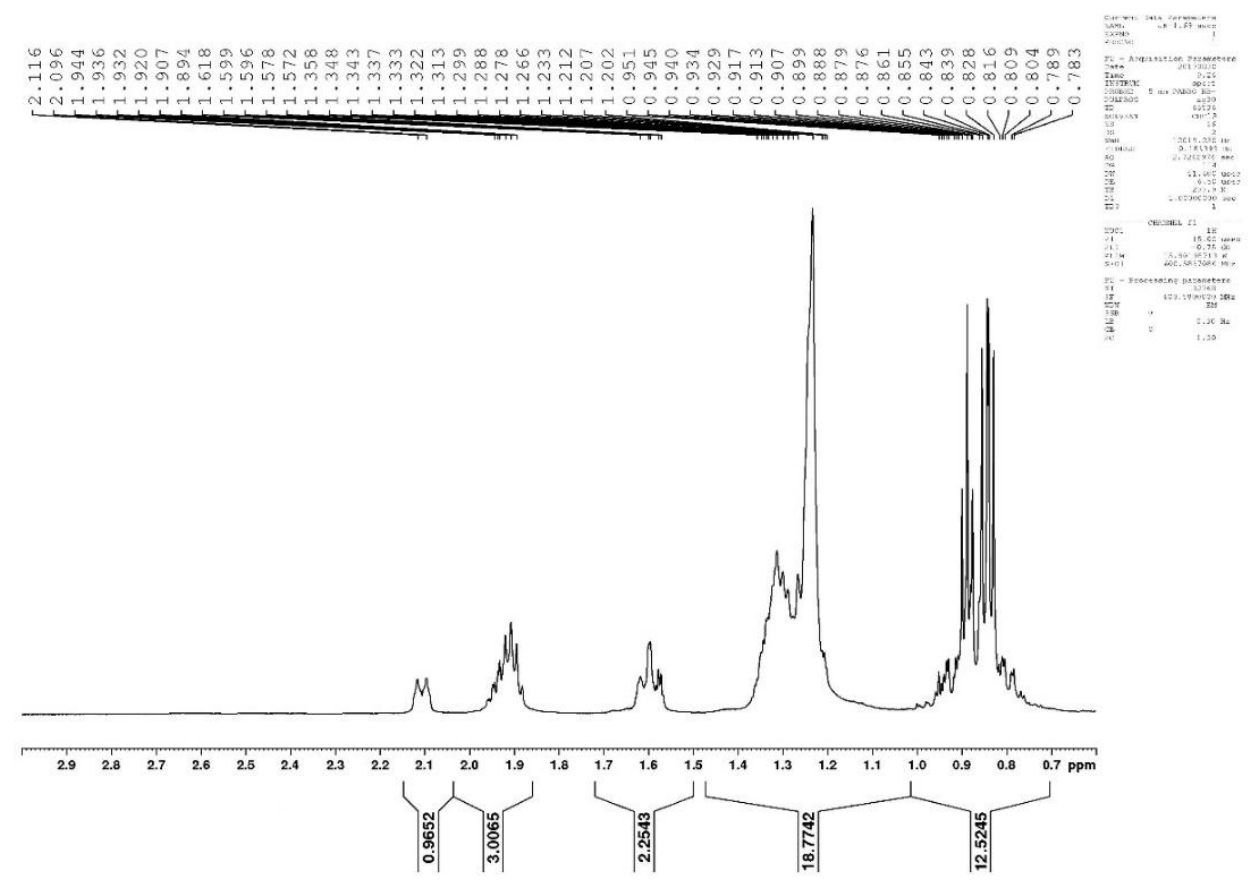

Figure S68. 


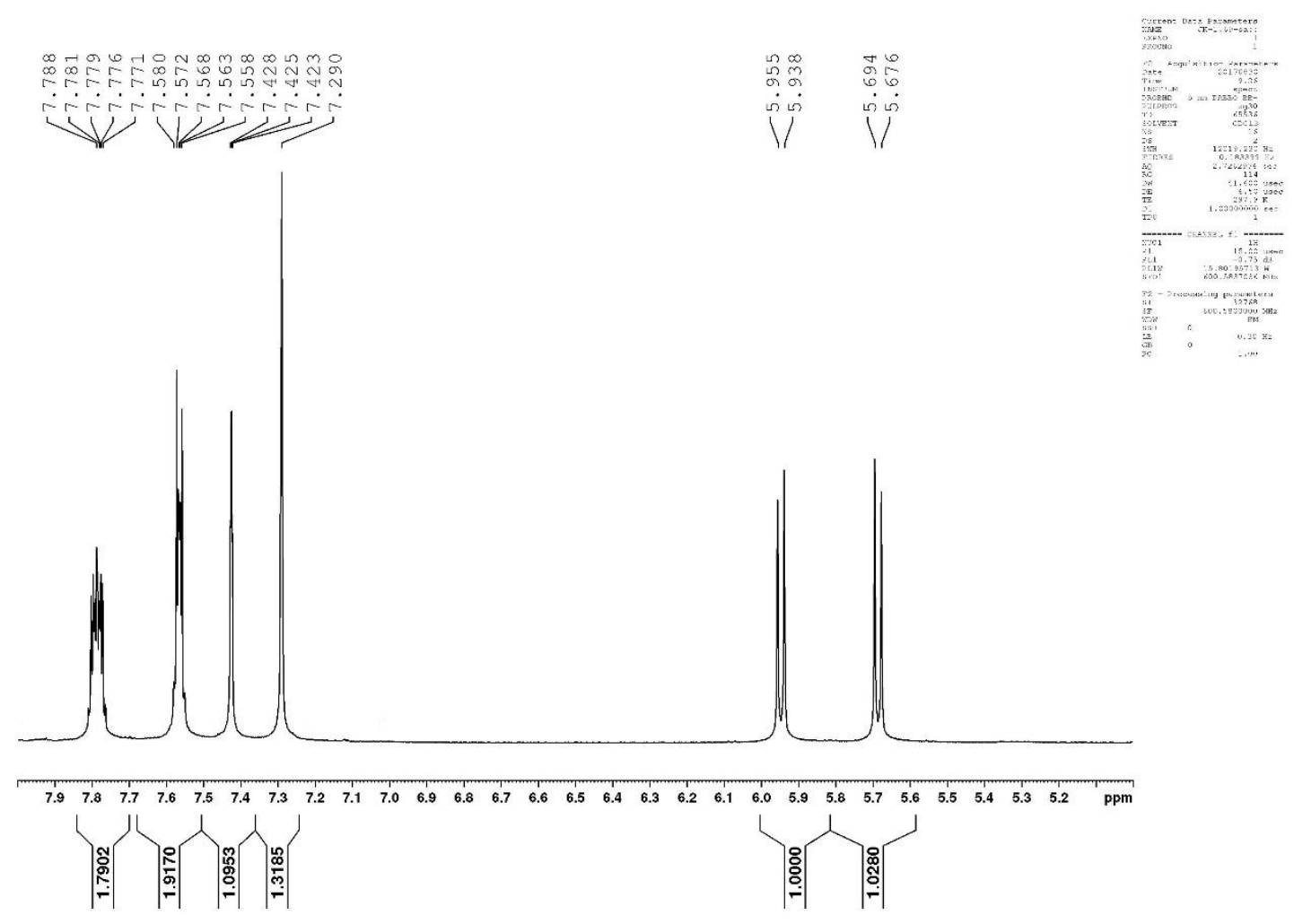

Figure S69. 
3-Dodecyl-1-[(1R.2S.5R)-(-)-menthoxymethyl]imidazolium saccharinate $\left[\mathrm{C}_{12}-\mathrm{Im}-\mathrm{CH}_{2} \mathrm{O}\right.$ Men][Sacc] (3I)

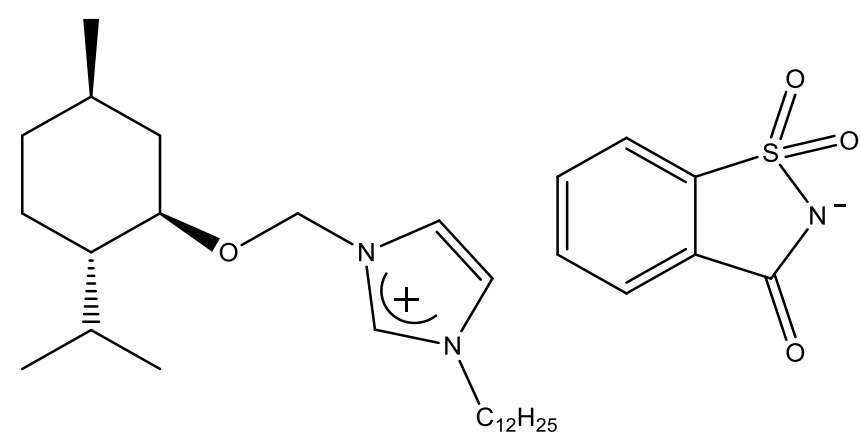
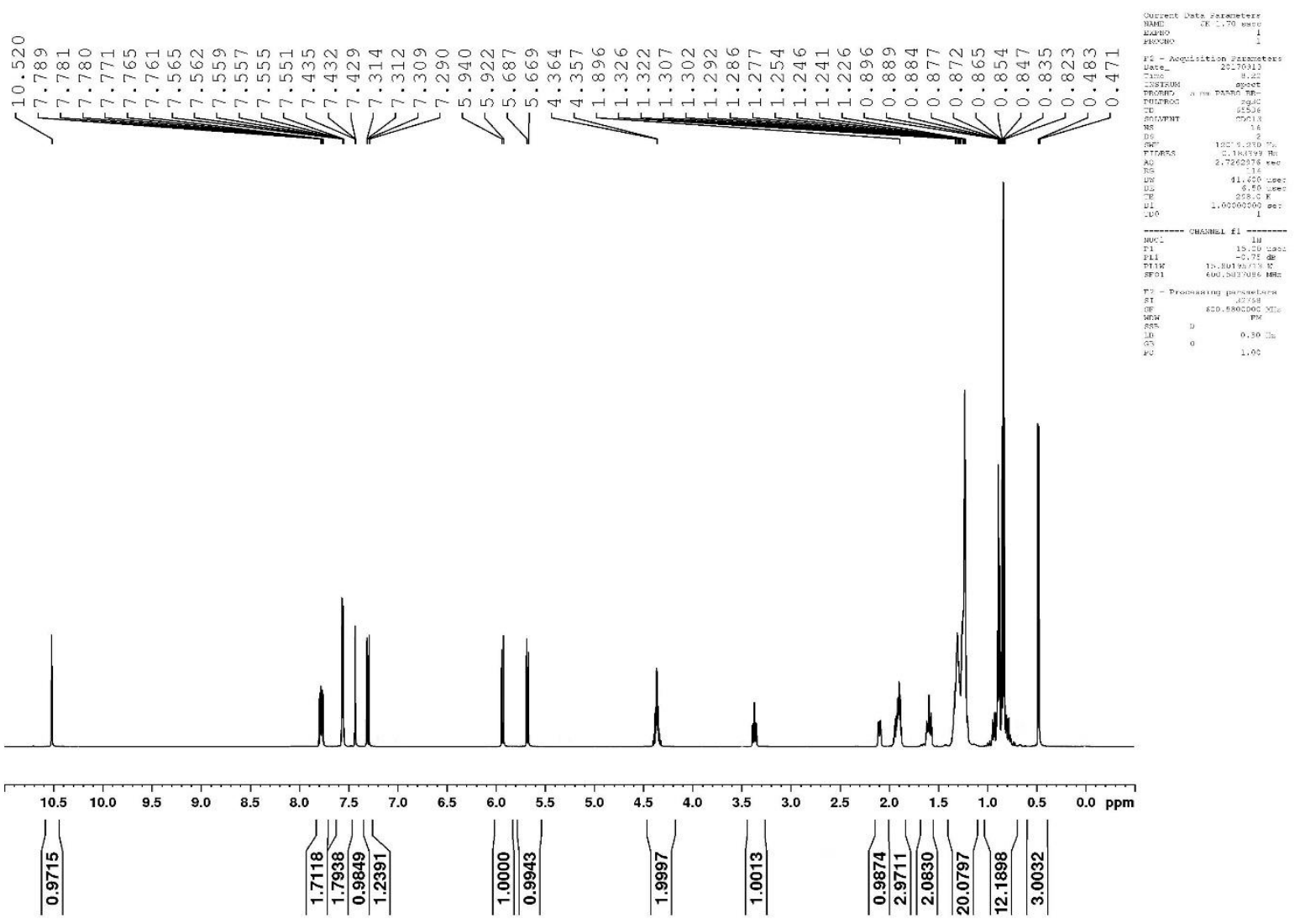

Figure S70. 


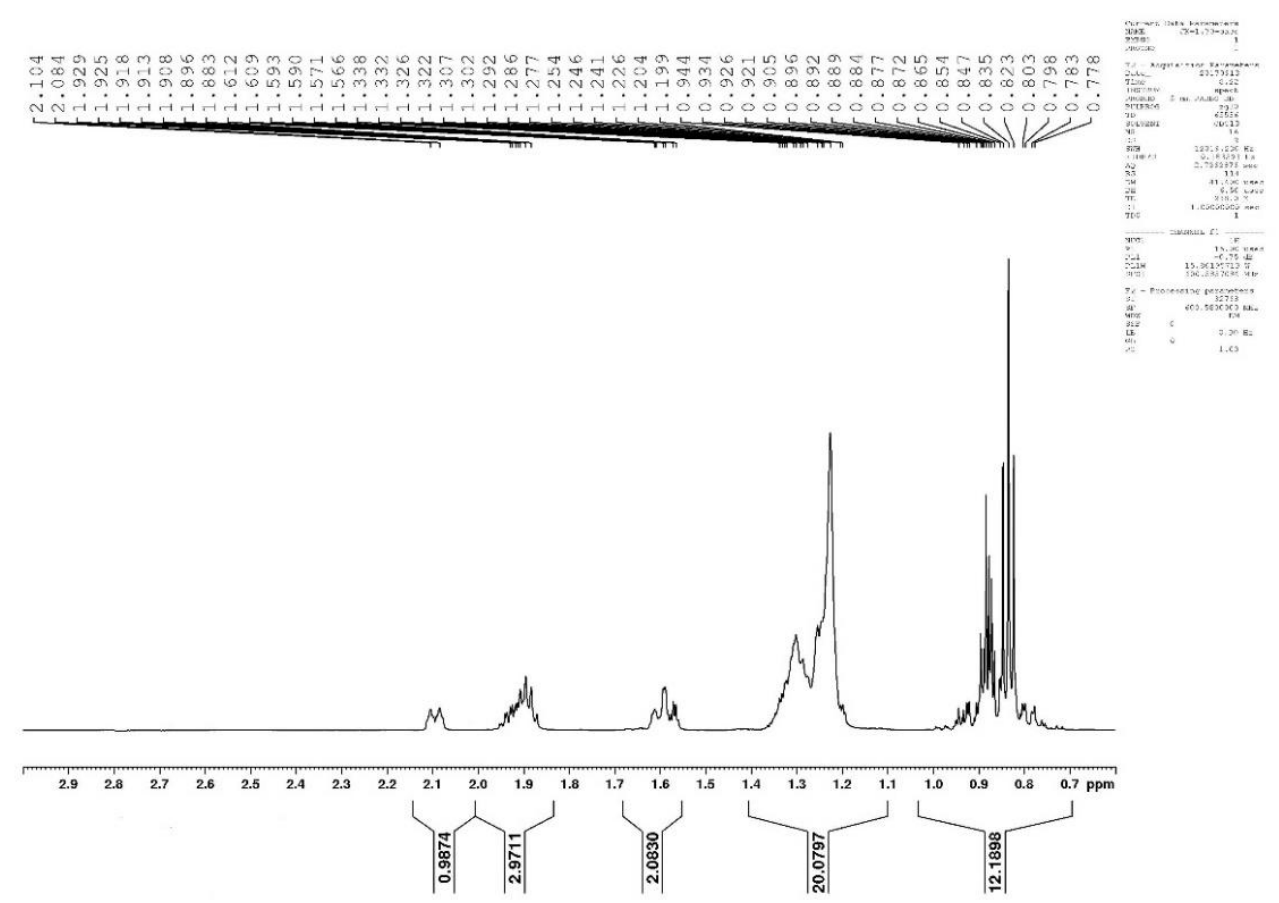

Figure S71.

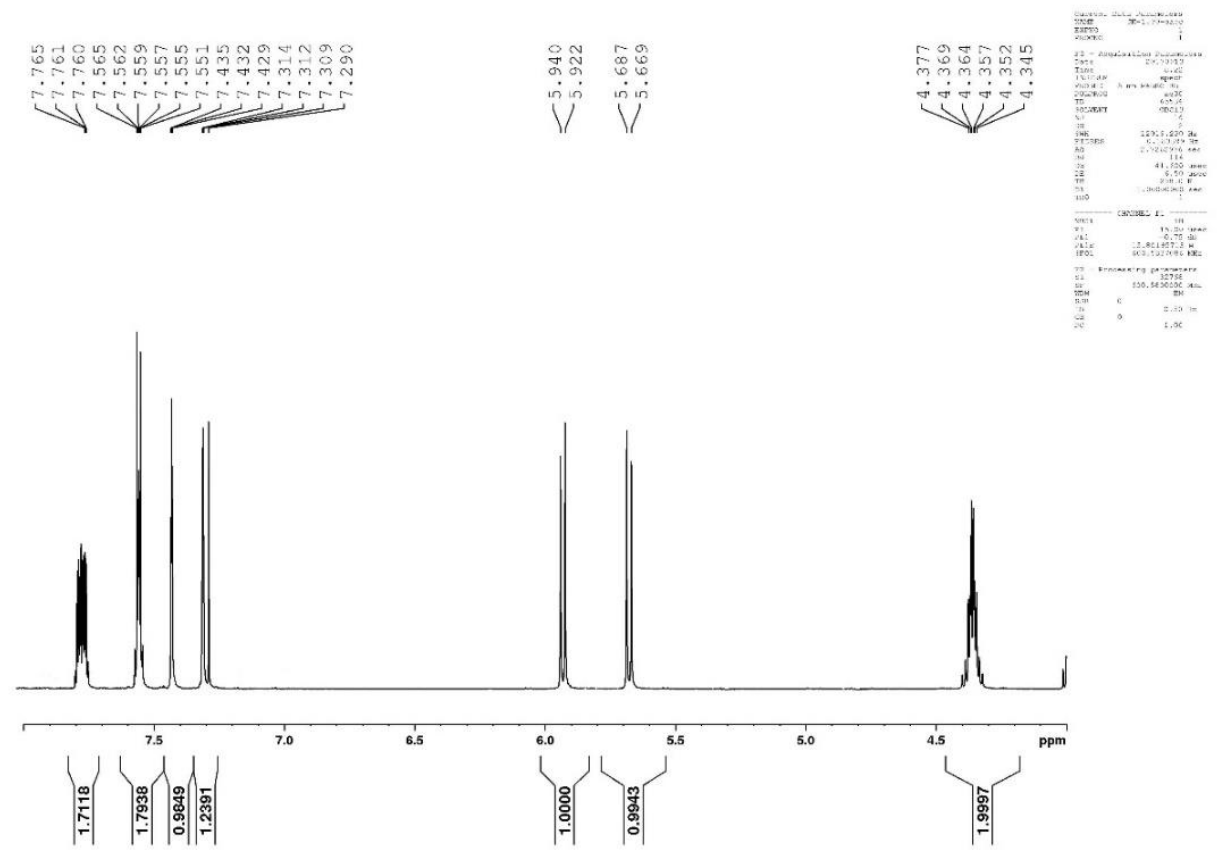

Figure S72. 


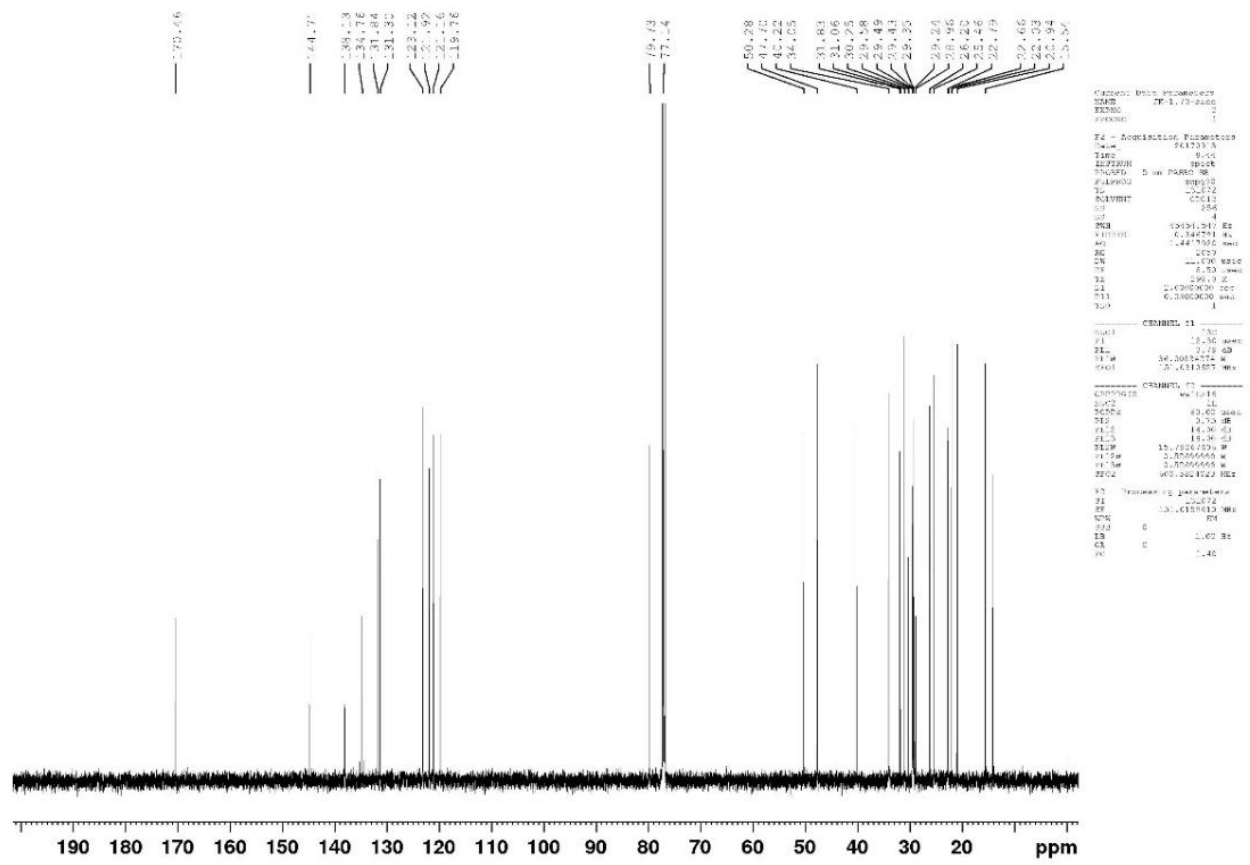

Figure S73.

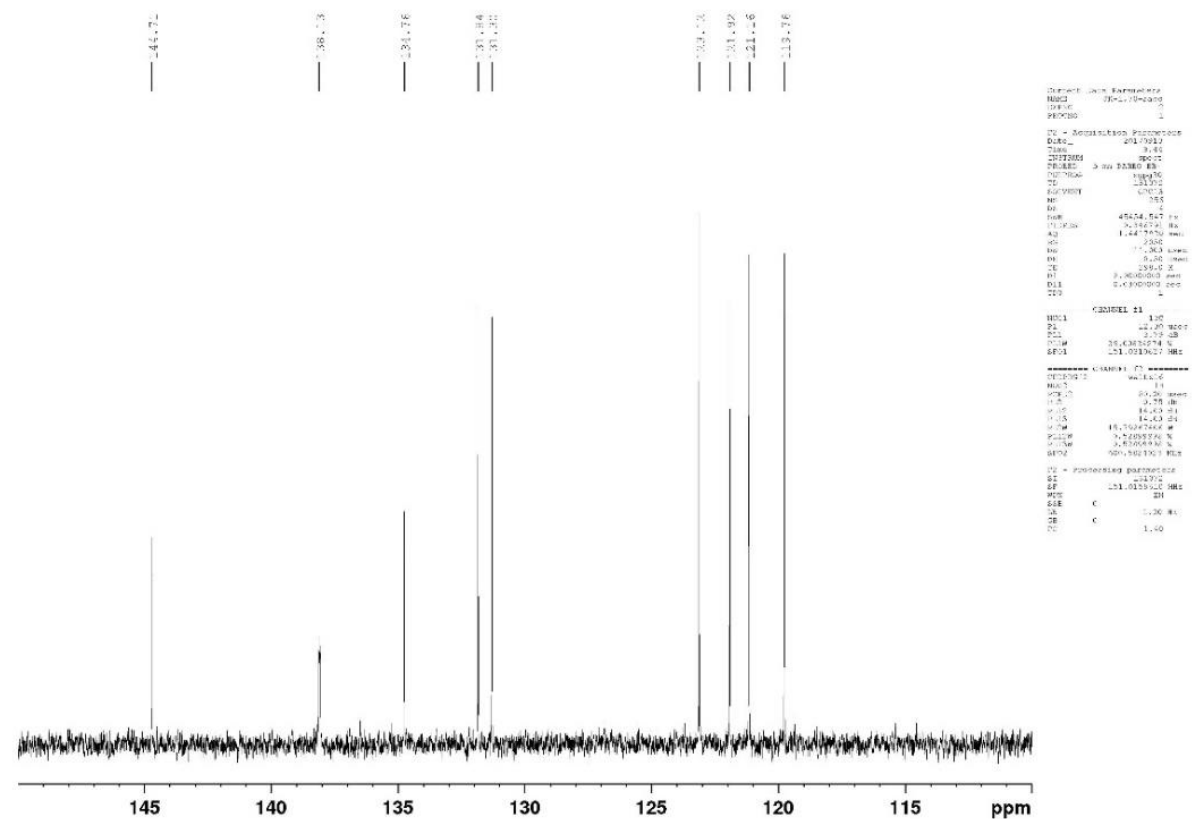

Figure S74. 


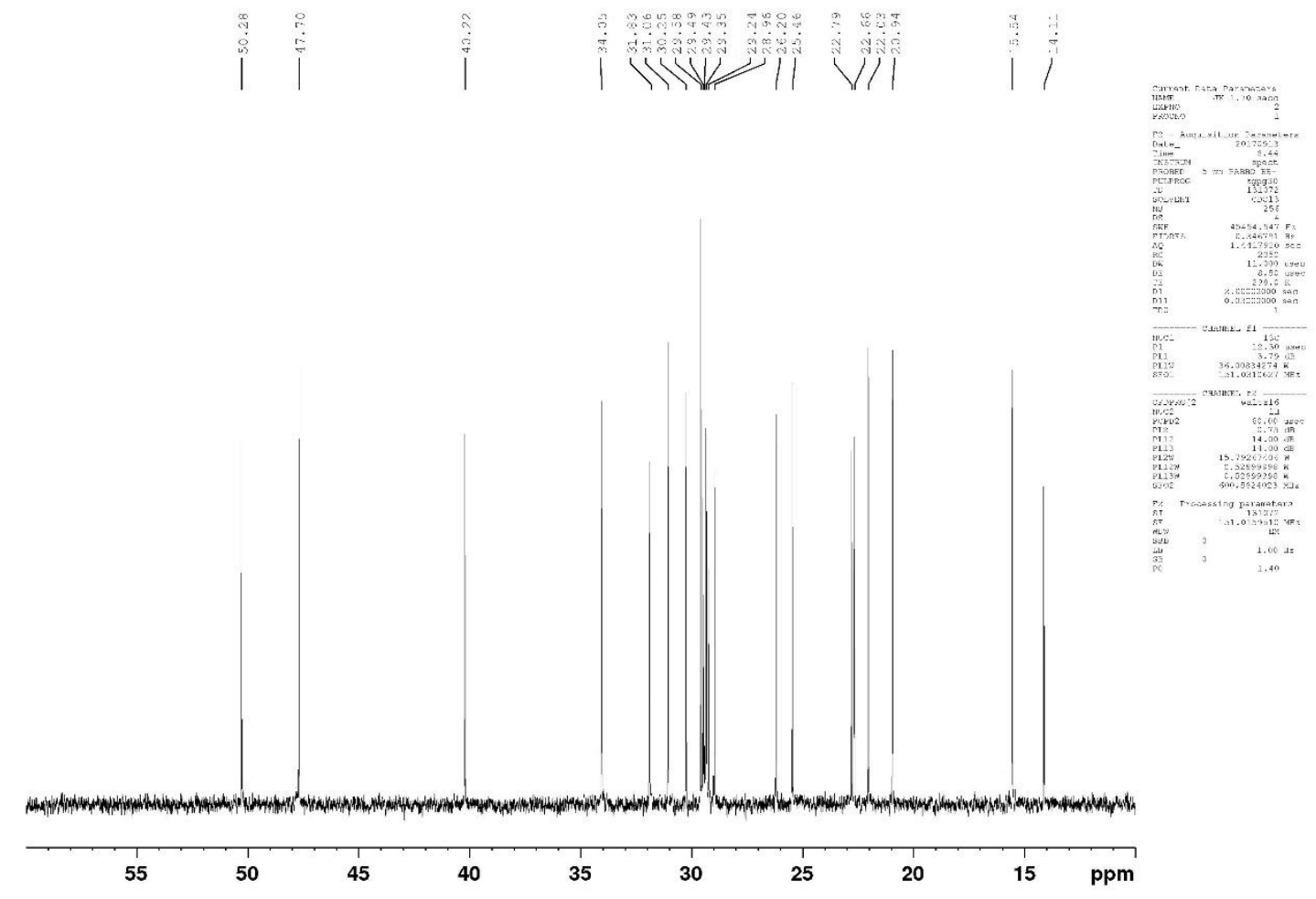

Figure S75. 


\section{REFERENCES}

(1) Skowronek, J.; Geppert-Rybczyńska, M.; Jacquemin, J.; Goodrich, P.; Alvarez Vicente, J.; Chorążewski, M.; Jężak, S.; Zorębski, M.; Zorębski, E.; Żarska, M.; Kaca, W.; Berdyczko, P.; Dzida, M. Acoustic and volumetric properties of diluted solutions of water in ionic liquids. J. Solution Chem. 2015, 44 (3-4), 824-837, DOI 10.1007/s10953-015-0327-z.

(2) EN ISO 2871-2 - EUROPEAN STANDARD 2010. Surface active agents. Detergents. Determination of cationic-active matter content. Part 2: Cationic-active matter of low molecular mass (between 200 and 500). Management Centre: Avenue Marnix 17, B-1000 Brussels.

(3) EN ISO 2871-1 - EUROPEAN STANDARD 2010. Surface active agents. Detergents. Determination of cationic-active matter content. Part 1: High-molecular-mass cationicactive matter. Management Centre: Avenue Marnix 17, B-1000 Brussels.

(4) Vogel, A. I.; Tatchell, A. R.; Furnis, B. S.; Hannaford, A. J.; Smith, P. W. G. Vogel's Textbook of Practical Organic Chemistry; Longman, $5^{\text {th }}$ ed, 1989.

(5) Geppert-Rybczyńska, M.; Lehmann, J. K.; Heintz, A. Volumetric properties of binary mixtures containing ionic liquids and some aprotic solvents. J. Chem. Eng. Data 2011, 56 (4), 1443-1448, DOI 10.1021/je101146f.

(6) Feder-Kubis, J.; Musiał, M.; Dzida, M.; Geppert-Rybczyńska, M. The new evolution of protic ionic liquids: antielectrostatic activity correlated with their surface properties. J. Ind. Eng. Chem. 2016, 41, 40-49, DOI 10.1016/j.jiec.2016.07.003.

(7) Carvalho, P. J.; Freire, M. G.; Marrucho, I. M.; Queimada, A. J.; Coutinho, J. A. P. Surface tensions for the 1-alkyl-3-methylimidazolium bis(trifluoromethylsulfonyl)imide ionic liquids. J. Chem. Eng. Data 2008, 53 (6), 1346-1350, DOI 10.1021/je800069z.

(8) Feder-Kubis, J.; Geppert-Rybczyńska, M.; Musiał, M.; Talik, E.; Guzik, A. Exploring the surface activity of a homologues series of functionalized ionic liquids with a natural chiral substituent: (-)-menthol in a cation. Colloids Surface A 2017, 529 (20), 725-732, DOI 10.1016/j.colsurfa.2017.06.040. 